Supporting Information for:

\title{
Automated Isotopic Profile Deconvolution for High Resolution Mass Spectrometric data (APGC-QToF) from Biological Matrices
}

Sadjad Fakouri Baygi, ${ }^{1}$ Sujan Fernando, ${ }^{2}$ Philip K. Hopke, ${ }^{1}$ Thomas M. Holsen, ${ }^{2,3}$ Bernard S. Crimmins $^{3,4 *}$

${ }^{1}$ Clarkson University, Department of Chemical and Biomolecular Engineering, 8 Clarkson Avenue, Potsdam, NY 13699, USA

${ }^{2}$ Clarkson University, Center for Air Resources Engineering and Science, 8 Clarkson Avenue, Potsdam, NY 13699, USA

${ }^{3}$ Clarkson University, Department of Civil and Environmental Engineering, 8 Clarkson Avenue, Potsdam, NY 13699, USA

${ }^{4}$ AEACS, LLC, New Kensington, PA 15068

* 8 Clarkson Avenue, CU Box 5708, Potsdam, NY 13699, Tel: 202-368-6926, Email: bcrimmin@clarkson.edu 


\section{Contents}

\section{Abbreviations}

\section{S.1. PBDEs standard}

Table S.1. The fragmentation pattern of PBDE congeners in negative polarity

Table S.2. The fragmentation pattern of PCB congeners in negative and positive APGC-QToF

Figure S.1. Comparison of the instrument response between positive and negative modes for with respect to the number of halogens for PCBs and PBDEs in Tables S.1 and S.2.

\section{S.2. Instrumentation}

\section{S.3. Isotope profile adjustment}

Table S.3. Comparison of Rectangular mass window and cluster merging method for $\mathrm{C}_{30} \mathrm{H}_{22} \mathrm{Br}_{5} \mathrm{Cl}_{5} \mathrm{~N}_{3} \mathrm{O}_{5} \mathrm{PS}_{2}$

Figure S.2. Comparison between rectangular mass window (RMW) and satellite cluster merging (SCM) methods for $\mathrm{C}_{30} \mathrm{H}_{22} \mathrm{Br}_{5} \mathrm{Cl}_{5} \mathrm{~N}_{3} \mathrm{O}_{5} \mathrm{PS}_{2}$ isotopic distribution profile calculation.

Table S.4. Isotope profile of $\mathrm{C}_{30} \mathrm{H}_{22} \mathrm{Br}_{5} \mathrm{Cl}_{5} \mathrm{~N}_{3} \mathrm{PO}_{5} \mathrm{~S}_{2}$

\section{S.4. Why the IPDC algorithm is computationally efficient for unknown compounds screening?}

\section{S.5. Similar Seed Profile Combination Example}

Table S.5. $\left\{\mathrm{C}_{10} \mathrm{Cl}_{9}\right\}$ seed profile comparison with average seed profile

Figure S.3. Flowchart of the IPDC algorithm

Table S.6. The criteria for the IPDC algorithm in positive and negative modes.

\section{S.6. Similar Isotope Profiles Example}

Figure S.4. Comparison of isotope profile of $\left[\mathrm{C}_{12} \mathrm{H}_{5} \mathrm{Br}_{2} \mathrm{O}_{2}\right]^{-}$and $\left[\mathrm{C}_{12} \mathrm{H}_{4} \mathrm{Cl}_{5} \mathrm{O}\right]^{-}$ions 
Figure S.5. Similarity of chromatogram traces of $\left[\mathrm{C}_{12} \mathrm{H}_{5} \mathrm{Br}_{2} \mathrm{O}_{2}\right]^{-}$and $\left[\mathrm{C}_{12} \mathrm{H}_{4} \mathrm{Cl}_{5} \mathrm{O}\right]^{-}$ions in PCB standard.

Table S.7. Comparison of identification of $\left[\mathrm{C}_{12} \mathrm{H}_{4} \mathrm{Cl}_{5} \mathrm{O}\right]^{-}$and $\left[\mathrm{C}_{12} \mathrm{H}_{5} \mathrm{Br}_{2} \mathrm{O}_{2}\right]^{-}$ions using the IPDC algorithm.

Figure S.6. Evaluation of search parameters used in Table 2 using PCB reference standards. Each point represents a chromatogram scan that contains PCB mass spectral peaks.

Figure S.7. Evaluation of mass error for the most abundant isotopologue used in molecular formulas assignment using PCB reference standards. Each point represents a chromatogram scan that contains PCB mass spectral peaks.

Figure S.8. Chromatographic peak analyses from IPDC algorithm results for a) PCB-81 and 77 in PCBs standard presented as $\left[\mathrm{C}_{12} \mathrm{H}_{6} \mathrm{Cl}_{3} \mathrm{O}\right]^{-}$ion, b) PCB-65 in LMLT 2016 presented as $\left[\mathrm{C}_{12} \mathrm{H}_{6} \mathrm{Cl}_{3} \mathrm{O}\right]^{-}$ion.

Figure S.9. False positive and true positive identifications analysis for four PCB standard mix injections. (a) True positive identifications, and (b) False positive identifications.

\section{S.9. Study the effect of weight of each parameter in the scoring formula}

Table S.8. Study weight of each parameter on overall score.

\section{S.10. Empirical equations for predicting boiling point of halogenated compounds}

Table S.9. Deviation of boiling point of PCBs and PBDEs from Equation (S.7)

Table S.10. Investigating Equation (6) on some halogenated compounds

\section{S.11. Estimating the impact of boiling point on retention time}

Figure S.10. Relationship of retention time and boiling point for some known halogenated compounds in Great Lakes Lake Trout.

\section{S.12. Column Bleed Filter}

Figure S.11. Mass defect plot for halogenated features 
Figure S.12. Detected halogenated features by the IPDC algorithm in the 20pg of PCB standard mixture in (a) negative and (b) positive modes.

Figure S.13. Isotopic features in LMLT 2016 composite in positive mode. The identifications were based on isotopic model confirmation and previous knowledge of the presence.

Table S.11. Summary of the IPDC algorithm results for the detected novel contaminants identified in LMLT 2014 (GC × GC-HRT) ${ }^{9}$ and in LMLT 2016 (Negative APGC-QToF). Additional molecular formula matches are provided for the negative APGC-QToF features. The negative APGC-QToF candidate that matches the molecular formula identified by the GC $\times$ GCToF is highlighted in green.

Table S.12. Summary of the IPDC algorithm results for the detected novel contaminants identified in LMLT 2014 (GC × GC-HRT) ${ }^{9}$ and in LMLT 2016 (Positive APGC-QToF). Additional molecular formula matches are provided for the positive APGC-QToF features. The positive APGC-QToF candidate that matches the molecular formula identified by the GC $\times$ GCToF is highlighted in blue.

Table S.13. Comparison between positive and negative APGC-QToF for the suggested novel contaminants in LMLT $2014(\mathrm{GC} \times \mathrm{GC}-\mathrm{HRT})^{9}$

\section{S.13. Checking Candidate Compounds against CompTox Chemicals Dashboard}

\section{References}




\begin{tabular}{|c|c|}
\hline APCI & Atmospheric pressure chemical ionization \\
\hline APGC-QToF & Atmospheric pressure gas chromatography-quadrupole time-of-flight \\
\hline EI & Electron impact \\
\hline ECNI & Electron capture negative ionization \\
\hline GC-MS & Gas chromatography mass spectrometry \\
\hline $\mathrm{GC} \times \mathrm{GC}-\mathrm{HRT}$ & 2-dimensional gas chromatograph coupled to a high resolution time-of-flight \\
\hline GLFMSP & Great lakes fish monitoring and surveillance program \\
\hline HRMS & High-resolution mass spectrometry \\
\hline IPDC & Isotopic profile deconvoluted chromatogram \\
\hline$\overline{\operatorname{Int}}$ & Clustered intensity \\
\hline$\overline{m / z}$ & Clustered average mass \\
\hline LMLT & Lake Michigan lake trout \\
\hline MLC & Machine learning classifier \\
\hline$N$ & Number of isotopologues in the isotopic model \\
\hline NEME & Normalized Euclidean mass error \\
\hline PCS & Profile cosine similarity \\
\hline$\overline{N E M E}$ & Normalized Euclidean mass error of the integrated spectra \\
\hline$\overline{P C S}$ & Profile cosine similarity of the integrated spectra \\
\hline NTS & Non-targeted screening \\
\hline MAIso & Most abundant isotopologue \\
\hline NDCS & Number of detected chromatogram scans \\
\hline $\mathrm{RCS}$ & Ratio of chromatogram scans \\
\hline RPW & Ratio of peak widths \\
\hline RMW & Rectangular mass window \\
\hline $\mathrm{SCM}$ & Satellite cluster merging \\
\hline PBDE & Polybrominated diphenyl ether \\
\hline PCB & Polychlorinated biphenyl \\
\hline PBT & Persistent, bioaccumulative, and/or toxic \\
\hline$w_{\text {Int }}$ & Peak width at intensity threshold \\
\hline$\delta_{t}$ & Retention time difference between two sequential chromatogram scans \\
\hline UPLC-QToF & Ultra-pressure liquid chromatography-quadrupole time-of-flight \\
\hline
\end{tabular}




\section{S.1. PBDEs standard}

BDE-MXE standard (Wellington Laboratories Inc., ON) fragmentation was investigated using a RTX-1614 $(15 \mathrm{~m} \times 250 \mu \mathrm{m} \times 0.1 \mu \mathrm{m})$ column (Restek, GA) as the GC separation column. The GC oven initial temperature was $120^{\circ} \mathrm{C}$, held for 1 minute and ramped to a temperature of $275^{\circ} \mathrm{C}$ at $15^{\circ} \mathrm{C} / \mathrm{min}$, and then increased to a final temperature of $330^{\circ} \mathrm{C}$ at $5^{\circ} \mathrm{C} / \mathrm{min}$ and held for 3 minutes for a total runtime of 25.33 minutes. The injector was operated in splitless mode at $300^{\circ} \mathrm{C}$. The mass spectrometer parameters and APGC source conditions are the same as detailed in the experimental section except the mass acquisition range was 50-1050 Da. The summary results are shown in Table S.1 for $1 \mu \mathrm{L}$ of stock solution injection. 
Table S.1. The fragmentation pattern of PBDE congeners in negative polarity

\begin{tabular}{|c|c|c|c|c|c|c|c|c|c|c|c|}
\hline \multirow{3}{*}{$\begin{array}{c}\text { PBDE } \\
\mathrm{C}_{12} \mathrm{H}_{9} \mathrm{BrO}\end{array}$} & \multirow{3}{*}{$\begin{array}{c}\begin{array}{c}\text { BDE } \\
\text { isomer } \\
\text { number }\end{array} \\
3\end{array}$} & \multicolumn{4}{|c|}{ The most abundant fragment } & \multicolumn{2}{|c|}{$[\mathbf{M}-\mathbf{B r}+\mathbf{O}]^{-}$} & \multicolumn{2}{|c|}{$[\mathbf{M}]^{-}$} & \multicolumn{2}{|c|}{$[\mathbf{M}]^{+}$} \\
\hline & & \multicolumn{2}{|c|}{ Fragment } & \multirow{2}{*}{$\begin{array}{c}\text { Mass }^{\mathbf{a}} \\
\text { ND }\end{array}$} & \multirow{2}{*}{$\begin{array}{c}\text { Intensity }^{\mathbf{b}} \\
\text { ND }\end{array}$} & \multirow{2}{*}{$\begin{array}{c}\text { Mass } \\
185.0602 \\
\end{array}$} & \multirow{2}{*}{$\begin{array}{c}\text { Relative } \\
\text { intensity } \\
(\%)^{c} \\
\text { ND }\end{array}$} & \multirow{2}{*}{$\begin{array}{c}\text { Mass } \\
247.9836\end{array}$} & \multirow{2}{*}{$\begin{array}{c}\begin{array}{c}\text { Relative } \\
\text { intensity } \\
(\%)^{\mathbf{d}}\end{array} \\
\text { ND }\end{array}$} & \multirow{2}{*}{$\begin{array}{c}\text { Mass } \\
247.9836\end{array}$} & \multirow{2}{*}{$\begin{array}{l}\text { Intensity }^{\mathbf{b}} \\
1.52 \mathrm{E}+06\end{array}$} \\
\hline & & ND & ND & & & & & & & & \\
\hline \multirow{2}{*}{$\mathrm{C}_{12} \mathrm{H}_{8} \mathrm{Br}_{2} \mathrm{O}$} & 7 & {$[\mathrm{M}-\mathrm{Br}+\mathrm{O}]^{-}$} & {$\left[\mathrm{C}_{12} \mathrm{H}_{8} \mathrm{BrO}_{2}\right]^{-}$} & 262.9708 & $3.23 \mathrm{E}+03$ & \multirow{2}{*}{262.9708} & 100 & \multirow{2}{*}{327.8922} & $\sim 0$ & \multirow{2}{*}{327.8922} & $1.08 \mathrm{E}+06$ \\
\hline & 15 & {$[\mathrm{M}-\mathrm{Br}+\mathrm{O}]^{-}$} & {$\left[\mathrm{C}_{12} \mathrm{H}_{8} \mathrm{BrO}_{2}\right]^{-}$} & 262.9708 & $8.78 \mathrm{E}+03$ & & 100 & & $\sim 0$ & & $1.07 \mathrm{E}+06$ \\
\hline \multirow{2}{*}{$\mathrm{C}_{12} \mathrm{H}_{7} \mathrm{Br}_{3} \mathrm{O}$} & 17 & {$\left[\mathrm{M}-\mathrm{H}-\mathrm{Br}_{2}+\mathrm{O}\right]^{-}$} & {$\left[\mathrm{C}_{12} \mathrm{H}_{6} \mathrm{BrO}_{2}\right]^{-}$} & 260.9551 & $1.45 \mathrm{E}+04$ & \multirow{2}{*}{342.8793} & 28.27 & \multirow{2}{*}{405.8027} & $\sim 0$ & \multirow{2}{*}{405.8027} & $7.36 \mathrm{E}+05$ \\
\hline & 28 & {$[\mathrm{M}-\mathrm{Br}+\mathrm{O}]^{-}$} & {$\left[\mathrm{C}_{12} \mathrm{H}_{7} \mathrm{Br}_{2} \mathrm{O}_{2}\right]^{-}$} & 342.8793 & $5.30 \mathrm{E}+04$ & & 100 & & $\sim 0$ & & $3.92 \mathrm{E}+05$ \\
\hline \multirow{4}{*}{$\mathrm{C}_{12} \mathrm{H}_{6} \mathrm{Br}_{4} \mathrm{O}$} & 49 & {$\left[\mathrm{M}-\mathrm{H}-\mathrm{Br}_{2}+\mathrm{O}\right]^{-}$} & {$\left[\mathrm{C}_{12} \mathrm{H}_{5} \mathrm{Br}_{2} \mathrm{O}_{2}\right]^{-}$} & 340.8636 & $1.89 \mathrm{E}+05$ & \multirow{4}{*}{420.7898} & 4.77 & \multirow{4}{*}{485.7112} & $\sim 0$ & \multirow{4}{*}{485.7112} & $1.01 \mathrm{E}+06$ \\
\hline & 71 & {$\left[\mathrm{M}-\mathrm{H}-\mathrm{Br}_{2}+\mathrm{O}\right]^{-}$} & {$\left[\mathrm{C}_{12} \mathrm{H}_{5} \mathrm{Br}_{2} \mathrm{O}_{2}\right]^{-}$} & 340.8636 & $2.39 \mathrm{E}+05$ & & 8.24 & & $\sim 0$ & & $7.50 \mathrm{E}+05$ \\
\hline & 47 & {$\left[\mathrm{M}-\mathrm{H}-\mathrm{Br}_{2}+\mathrm{O}\right]^{-}$} & {$\left[\mathrm{C}_{12} \mathrm{H}_{5} \mathrm{Br}_{2} \mathrm{O}_{2}\right]^{-}$} & 340.8636 & $1.60 \mathrm{E}+05$ & & 3.06 & & $\sim 0$ & & $6.20 \mathrm{E}+05$ \\
\hline & 77 & {$[\mathrm{M}-\mathrm{Br}+\mathrm{O}]^{-}$} & {$\left[\mathrm{C}_{12} \mathrm{H}_{6} \mathrm{Br}_{3} \mathrm{O}_{2}\right]^{-}$} & 420.7898 & $7.32 \mathrm{E}+04$ & & 100 & & $\sim 0$ & & $9.01 \mathrm{E}+04$ \\
\hline \multirow{5}{*}{$\mathrm{C}_{12} \mathrm{H}_{5} \mathrm{Br}_{5} \mathrm{O}$} & 100 & {$\left[\mathrm{M}-\mathrm{H}-\mathrm{Br}_{2}+\mathrm{O}\right]^{-}$} & {$\left[\mathrm{C}_{12} \mathrm{H}_{4} \mathrm{Br}_{3} \mathrm{O}_{2}\right]^{-}$} & 418.7741 & $1.19 \mathrm{E}+06$ & \multirow{5}{*}{500.6983} & 0.73 & \multirow{5}{*}{563.6217} & $\sim 0$ & \multirow{5}{*}{563.6217} & $5.06 \mathrm{E}+05$ \\
\hline & 119 & {$\left[\mathrm{M}-\mathrm{H}-\mathrm{Br}_{2}+\mathrm{O}\right]^{-}$} & {$\left[\mathrm{C}_{12} \mathrm{H}_{4} \mathrm{Br}_{3} \mathrm{O}_{2}\right]^{-}$} & 418.7741 & $1.12 \mathrm{E}+06$ & & 0.61 & & $\sim 0$ & & $7.53 \mathrm{E}+05$ \\
\hline & 99 & {$\left[\mathrm{M}-\mathrm{H}-\mathrm{Br}_{2}+\mathrm{O}\right]^{-}$} & {$\left[\mathrm{C}_{12} \mathrm{H}_{4} \mathrm{Br}_{3} \mathrm{O}_{2}\right]^{-}$} & 418.7741 & $3.69 \mathrm{E}+04$ & & 7.97 & & $\sim 0$ & & $5.87 \mathrm{E}+05$ \\
\hline & 85 & {$\left[\mathrm{M}-\mathrm{H}-\mathrm{Br}_{2}+\mathrm{O}\right]^{-}$} & {$\left[\mathrm{C}_{12} \mathrm{H}_{4} \mathrm{Br}_{3} \mathrm{O}_{2}\right]^{-}$} & 418.7741 & $4.75 \mathrm{E}+05$ & & 1.04 & & $\sim 0$ & & $5.70 \mathrm{E}+05$ \\
\hline & 126 & {$[\mathrm{M}-\mathrm{Br}+\mathrm{O}]^{-}$} & {$\left[\mathrm{C}_{12} \mathrm{H}_{5} \mathrm{Br}_{4} \mathrm{O}_{2}\right]^{-}$} & 500.6983 & $1.69 \mathrm{E}+05$ & & 100 & & $\sim 0$ & & $4.18 \mathrm{E}+05$ \\
\hline $\mathrm{C}_{12} \mathrm{H}_{4} \mathrm{Br}_{6} \mathrm{O}$ & 154 & {$\left[\mathrm{M}-\mathrm{H}-\mathrm{Br}_{2}+\mathrm{O}\right]^{-}$} & {$\left[\mathrm{C}_{12} \mathrm{H}_{3} \mathrm{Br}_{4} \mathrm{O}_{2}\right]^{-}$} & 498.6826 & $2.53 \mathrm{E}+06$ & 578.6088 & 1.47 & 643.5302 & 0.01 & 643.5302 & $1.44 \mathrm{E}+06$ \\
\hline
\end{tabular}




\begin{tabular}{|c|c|c|c|c|c|c|c|c|c|c|c|}
\hline & 153 & {$\left[\mathrm{M}-\mathrm{H}-\mathrm{Br}_{2}+\mathrm{O}\right]^{-}$} & {$\left[\mathrm{C}_{12} \mathrm{H}_{3} \mathrm{Br}_{4} \mathrm{O}_{2}\right]^{-}$} & 498.6826 & $2.93 \mathrm{E}+06$ & & 1.83 & & 1.12 & & $1.11 \mathrm{E}+06$ \\
\hline & 138 & {$\left[\mathrm{M}-\mathrm{H}-\mathrm{Br}_{2}+\mathrm{O}\right]^{-}$} & {$\left[\mathrm{C}_{12} \mathrm{H}_{3} \mathrm{Br}_{4} \mathrm{O}_{2}\right]^{-}$} & 498.6826 & $9.63 \mathrm{E}+05$ & & 2.85 & & 0.2 & & $1.10 \mathrm{E}+05$ \\
\hline & 156 & {$\left[\mathrm{M}-\mathrm{H}-\mathrm{Br}_{2}+\mathrm{O}\right]^{-}$} & {$\left[\mathrm{C}_{12} \mathrm{H}_{3} \mathrm{Br}_{4} \mathrm{O}_{2}\right]^{-}$} & 498.6826 & $6.59 \mathrm{E}+05$ & & 22.91 & & 0.25 & & $7.75 \mathrm{E}+05$ \\
\hline \multirow{3}{*}{$\mathrm{C}_{12} \mathrm{H}_{3} \mathrm{Br}_{7} \mathrm{O}$} & 184 & {$\left[\mathrm{M}-\mathrm{C}_{6} \mathrm{H}_{2} \mathrm{Br}_{3}\right]^{-}$} & {$\left[\mathrm{C}_{6} \mathrm{HBr}_{4} \mathrm{O}\right]^{-}$} & 408.672 & $1.74 \mathrm{E}+06$ & \multirow{3}{*}{658.5173} & 4.41 & \multirow{3}{*}{721.4407} & 6.83 & \multirow{3}{*}{721.4407} & $1.03 \mathrm{E}+05$ \\
\hline & 183 & {$[\mathrm{M}-\mathrm{H}-\mathrm{Br}]^{-}$} & {$\left[\mathrm{C}_{12} \mathrm{H}_{2} \mathrm{Br}_{5} \mathrm{O}_{2}\right]^{-}$} & 576.5931 & $2.00 \mathrm{E}+05$ & & 31.05 & & 3.9 & & $7.07 \mathrm{E}+05$ \\
\hline & 191 & {$[\mathrm{M}-\mathrm{H}-\mathrm{Br}]^{-}$} & {$\left[\mathrm{C}_{12} \mathrm{H}_{2} \mathrm{Br}_{5} \mathrm{O}_{2}\right]^{-}$} & 576.5931 & $8.31 \mathrm{E}+04$ & & 71.23 & & 8.1 & & $6.35 \mathrm{E}+05$ \\
\hline \multirow{2}{*}{$\mathrm{C}_{12} \mathrm{H}_{2} \mathrm{Br}_{8} \mathrm{O}$} & 197 & {$\left[\mathrm{M}-\mathrm{C}_{6} \mathrm{HBr}_{4}\right]^{-}$} & {$\left[\mathrm{C}_{6} \mathrm{HBr}_{4} \mathrm{O}\right]^{-}$} & 408.672 & $1.70 \mathrm{E}+06$ & \multirow{2}{*}{736.4277} & 0.56 & \multirow{2}{*}{801.3491} & 4.59 & \multirow{2}{*}{801.3491} & $4.16 \mathrm{E}+05$ \\
\hline & 196 & {$[\mathrm{M}-\mathrm{H}-\mathrm{Br}]^{-}$} & {$\left[\mathrm{C}_{12} \mathrm{HBr}_{7} \mathrm{O}\right]^{-}$} & 719.425 & $2.12 \mathrm{E}+05$ & & 10.7 & & 1.78 & & $3.48 \mathrm{E}+05$ \\
\hline \multirow{2}{*}{$\mathrm{C}_{12} \mathrm{HBr}_{9} \mathrm{O}$} & 207 & {$\left[\mathrm{M}-\mathrm{C}_{6} \mathrm{HBr}_{4}\right]^{-}$} & {$\left[\mathrm{C}_{6} \mathrm{Br}_{5} \mathrm{O}\right]^{-}$} & 486.5825 & $3.67 \mathrm{E}+06$ & \multirow{2}{*}{816.3362} & 1.29 & \multirow{2}{*}{879.2596} & 5.42 & \multirow{2}{*}{879.2596} & $3.74 \mathrm{E}+05$ \\
\hline & 206 & {$[\mathrm{M}-\mathrm{H}-\mathrm{Br}]^{-}$} & {$\left[\mathrm{C}_{12} \mathrm{Br}_{8} \mathrm{O}\right]^{-}$} & 799.3335 & $9.33 \mathrm{E}+05$ & & 74.7 & & 35.37 & & $2.59 \mathrm{E}+05$ \\
\hline $\mathrm{C}_{12} \mathrm{Br}_{10} \mathrm{O}$ & 209 & {$\left[\mathrm{M}-\mathrm{C}_{6} \mathrm{Br}_{5}\right]^{-}$} & {$\left[\mathrm{C}_{6} \mathrm{Br}_{5} \mathrm{O}\right]^{-}$} & 486.5825 & $2.62 \mathrm{E}+06$ & 894.2468 & 2.5 & 959.1682 & 0.88 & 959.1682 & $1.04 \mathrm{E}+05$ \\
\hline
\end{tabular}


Table S.2. The fragmentation pattern of PCB congeners in negative and positive APGC-QToF

\begin{tabular}{|c|c|c|c|c|c|c|c|c|c|}
\hline \multirow[b]{2}{*}{ PCB } & \multirow[b]{2}{*}{$\begin{array}{c}\text { PCB } \\
\text { isomer } \\
\text { number }\end{array}$} & \multicolumn{2}{|c|}{$[\mathrm{M}-\mathrm{Cl}+\mathbf{O}]^{-}$} & \multicolumn{2}{|c|}{$[\mathbf{M}]^{-}$} & \multicolumn{2}{|c|}{$\left[\mathrm{M}-\mathrm{H}-\mathrm{Cl}+\mathrm{O}_{2}\right]^{-}$} & \multicolumn{2}{|c|}{$[\mathbf{M}]^{+}$} \\
\hline & & Mass $^{\mathbf{a}}$ & Intensity $^{b}$ & Mass & $\begin{array}{c}\text { Relative } \\
\text { intensity } \\
(\%)^{c}\end{array}$ & Mass & $\begin{array}{c}\text { Relative } \\
\text { intensity } \\
(\%)^{d}\end{array}$ & Mass & Intensity a \\
\hline \multirow{2}{*}{$\mathrm{C}_{12} \mathrm{H}_{9} \mathrm{Cl}$} & 1 & \multirow{2}{*}{169.0653} & ND & \multirow{2}{*}{188.0392} & ND & \multirow{2}{*}{184.0524} & ND & \multirow{2}{*}{188.0392} & $1.81 \mathrm{E}+03$ \\
\hline & 3 & & ND & & ND & & ND & & $3.96 \mathrm{E}+03$ \\
\hline \multirow{2}{*}{$\mathrm{C}_{12} \mathrm{H}_{8} \mathrm{Cl}_{2}$} & 4 & \multirow{2}{*}{203.0263} & ND & \multirow{2}{*}{222.0003} & ND & \multirow{2}{*}{218.0134} & ND & \multirow{2}{*}{222.0003} & $1.43 \mathrm{E}+03$ \\
\hline & 15 & & ND & & ND & & ND & & $3.84 \mathrm{E}+03$ \\
\hline \multirow{2}{*}{$\mathrm{C}_{12} \mathrm{H}_{7} \mathrm{Cl}_{3}$} & 19 & \multirow{2}{*}{236.9874} & ND & \multirow{2}{*}{255.9613} & $\sim 0$ & \multirow{2}{*}{251.9745} & $\sim 0$ & \multirow{2}{*}{255.9613} & $1.69 \mathrm{E}+03$ \\
\hline & 37 & & $5.09 \mathrm{E}+02$ & & $\sim 0$ & & $\sim 0$ & & $2.89 \mathrm{E}+03$ \\
\hline \multirow{3}{*}{$\mathrm{C}_{12} \mathrm{H}_{6} \mathrm{Cl}_{4}$} & 54 & \multirow{3}{*}{270.9484} & $\sim 0$ & \multirow{3}{*}{291.9194} & $\sim 0$ & \multirow{3}{*}{285.9355} & $\sim 0$ & \multirow{3}{*}{291.9194} & $2.59 \mathrm{E}+03$ \\
\hline & 81 & & $7.34 \mathrm{E}+03$ & & $\sim 0$ & & 29.83 & & $5.39 \mathrm{E}+03$ \\
\hline & 77 & & $4.30 \mathrm{E}+03$ & & $\sim 0$ & & $\sim 0$ & & $3.91 \mathrm{E}+03$ \\
\hline \multirow{6}{*}{$\mathrm{C}_{12} \mathrm{H}_{5} \mathrm{Cl}_{5}$} & 104 & \multirow{6}{*}{306.9065} & $4.16 \mathrm{E}+02$ & \multirow{6}{*}{325.8805} & $\sim 0$ & \multirow{6}{*}{321.8936} & $\sim 0$ & & $6.22 \mathrm{E}+03$ \\
\hline & 123 & & $2.28 \mathrm{E}+04$ & & $\sim 0$ & & 13.51 & & $6.60 \mathrm{E}+03$ \\
\hline & 118 & & $3.51 \mathrm{E}+04$ & & $\sim 0$ & & 8.77 & & $5.18 \mathrm{E}+03$ \\
\hline & 114 & & $2.93 \mathrm{E}+04$ & & $\sim 0$ & & 10.51 & 325.8805 & $4.73 \mathrm{E}+03$ \\
\hline & 105 & & $2.28 \mathrm{E}+04$ & & $\sim 0$ & & 13.51 & & $4.59 \mathrm{E}+03$ \\
\hline & 126 & & $1.55 \mathrm{E}+04$ & & $\sim 0$ & & 19.87 & & $5.52 \mathrm{E}+03$ \\
\hline & 155 & & $1.20 \mathrm{E}+03$ & & $\sim 0$ & & $\sim 0$ & & $4.22 \mathrm{E}+03$ \\
\hline & 167 & & $3.76 \mathrm{E}+04$ & & $\sim 0$ & & 8.19 & & $4.58 \mathrm{E}+03$ \\
\hline $\mathrm{C}_{12} \mathrm{H}_{4} \mathrm{Cl}_{6}$ & 156 & 340.8675 & $3.67 \mathrm{E}+04$ & 359.8415 & $\sim 0$ & 355.8546 & 8.39 & 359.8415 & $4.16 \mathrm{E}+03$ \\
\hline & 157 & & $3.28 \mathrm{E}+04$ & & $\sim 0$ & & 9.39 & & $4.29 \mathrm{E}+03$ \\
\hline & 169 & & $2.11 \mathrm{E}+04$ & & $\sim 0$ & & 14.60 & & $4.05 \mathrm{E}+03$ \\
\hline & 188 & 37 & $2.35 \mathrm{E}+04$ & & $\sim 0$ & & $\sim 0$ & & $3.77 \mathrm{E}+03$ \\
\hline $\mathrm{CI}_{7}$ & 189 & $3 / 4.8286$ & $3.79 \mathrm{E}+04$ & 393.8025 & $\sim 0$ & 389.8156 & 8.13 & 393.8025 & $3.56 \mathrm{E}+03$ \\
\hline & 202 & & $2.97 \mathrm{E}+04$ & & $\sim 0$ & & $\sim 0$ & & $3.15 \mathrm{E}+03$ \\
\hline $\mathrm{C}_{12} \mathrm{H}_{2} \mathrm{Cl}_{8}$ & 205 & 408.7896 & $2.36 \mathrm{E}+04$ & 429.7606 & 44.06 & $423.7 / 67$ & $\sim 0$ & 429.7606 & $2.98 \mathrm{E}+03$ \\
\hline & 208 & & $2.24 \mathrm{E}+04$ & & 4.21 & 348 & $\sim 0$ & & $2.38 \mathrm{E}+03$ \\
\hline $\mathrm{C}_{12} \mathrm{HCl}_{9}$ & 206 & 444.7477 & $2.12 \mathrm{E}+04$ & 216 & 4.54 & 348 & $\sim 0$ & 216 & $2.59 \mathrm{E}+03$ \\
\hline $\mathrm{C}_{12} \mathrm{Cl}_{10}$ & 209 & 478.7087 & $1.03 \mathrm{E}+04$ & 497.6826 & 4.44 & NA & NA & 497.6826 & $2.42 \mathrm{E}+03$ \\
\hline
\end{tabular}

${ }^{a}$ Mass of the most abundant isotopologue

${ }^{b}$ Intensity of the peak in continuum mode

$c, \mathrm{~d}$ Relative intensity respect to the intensity of $[\mathrm{M}-\mathrm{Cl}+\mathrm{O}]^{-}$ions 


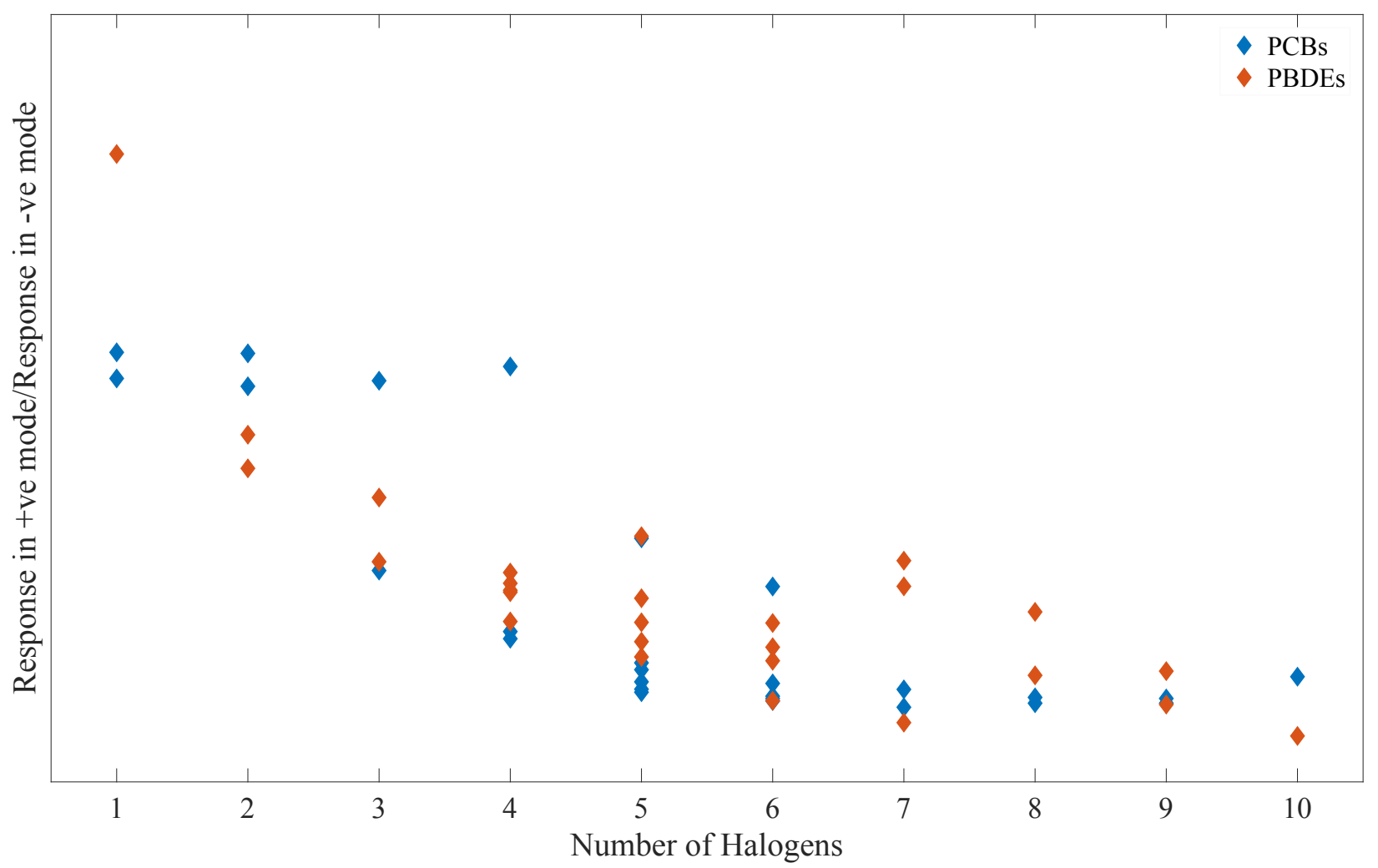

Figure S.1. Comparison of the instrument response between positive and negative modes for with respect to the number of halogens for PCBs and PBDEs in Tables S.1 and S.2. 


\section{S.2. Instrumentation}

The extract was injected $(1 \mu \mathrm{L})$ and analyzed using a hybrid analytical instrument consisting of an Agilent 7890A GC connected to a Waters Xevo G2-XS QToF through an APGC interface. The GC was fitted with an Rxi-5ms (Restek, GA) or J\&W DB-5ms (Agilent, CA) capillary column $(30 \mathrm{~m} \times 250 \mu \mathrm{m} \times 0.25 \mu \mathrm{m})$. A $40-50 \mathrm{~cm}$ deactivated silica column was used in the transfer line from the GC to the APGC source. The transfer line was maintained at $350^{\circ} \mathrm{C}$ throughout the run. The GC oven initial temperature was set to $40^{\circ} \mathrm{C}$, held for 1 minute and ramped to final temperature of $300^{\circ} \mathrm{C}$ at $5^{\circ} \mathrm{C} / \mathrm{min}$, and then held for 25 minutes for a total runtime of 78 minutes. The injector is operated in temperature programmed splitless mode, with an initial temperature of $50^{\circ} \mathrm{C}$ and then increased to $300^{\circ} \mathrm{C}$ at $150^{\circ} \mathrm{C} / \mathrm{min}$ as soon as the injection is completed.

The mass spectrometer was calibrated immediately before the experiments using perfluorotributylamine (PFTBA) resulting in a mass accuracy better than $1 \mathrm{mDa}$ and resolution of 22,000 FWHM. During the analysis, methanol (only in negative mode) and PFTBA were continuously bled into the source region. Masses 451.9749 Da and 501.9711Da from PFTBA was used for the lockmass correction to compensate for mass accuracy fluctuations during the chromatographic run in negative and positive modes, respectively. The APGC source parameters in both modes are as follows: source temperature at $150^{\circ} \mathrm{C}$, cone gas at $150 \mathrm{~L} / \mathrm{h}$ and auxillary gas at $100 \mathrm{~L} / \mathrm{h}$. Likewise, corona current at $5 \mu \mathrm{A}$ and $10 \mu \mathrm{A}$, sampling cone at $40 \mathrm{~V}$ and $20 \mathrm{~V}$ were set for negative and positive modes, respectively. Continuum mass spectral data are acquired in alternating low and high energy channels known as $\mathrm{MS}^{\mathrm{e}}$ mode ${ }^{1}$ in sensitivity mode with a scan range of 50-950 Da at $0.13 \mathrm{~s}^{-1}$. The optimum scan rate for the IPDC algorithm is recommended to be between $0.1-0.15 \mathrm{~s}^{-1}$. 


\section{S.3. Isotope profile adjustment}

When a compound contains several elements with different isotopic abundances, some isotopologue peaks may exist in a small mass range that may be smaller than the instrument's resolution. Neighboring isotopologues within the instrument resolution were clustered and resolved into a single peak. The satellite cluster merging (SCM) method clusters isotopologues with the nearest abundant isotopologue that is within the instrument resolution range and subsequently all isotopologues in the cluster are merged into a single isotopologue ${ }^{2}$. The SCM method was chosen over the previously used rectangular mass window (RMW) method because the RMW is not applicable when the exact mass of the compound is not available. ${ }^{3} \mathrm{SCM}$ and RMW methods may result in similar or different isotope profile depending on the instrument resolution. The maximum difference between two isotopologues is $1 \mathrm{mDa}$ according to the instrument calibration and resolution.

The isotopic distribution profile using RMW and SCM methods for $\mathrm{C}_{30} \mathrm{H}_{22} \mathrm{Br}_{5} \mathrm{Cl}_{5} \mathrm{~N}_{3} \mathrm{O}_{5} \mathrm{PS}_{2}$, the most complex molecular formula considered in this study, is shown in Table S.3 and Figure S.1. The SCM method resolved more neighboring isotopologues and resulted in a profile with a smaller number of isotopologues. However, the corresponding isotopologues (Table S.3) shows small discrepancies (maximum $0.51 \mathrm{mDa}$ mass difference and $99.83 \%$ profile cosine similarity) indicating the SCM method is similar to the RMW approach used previously. 
Table S.3. Comparison of Rectangular mass window and cluster merging method for $\mathrm{C}_{30} \mathrm{H}_{22} \mathrm{Br}_{5} \mathrm{Cl}_{5} \mathrm{~N}_{3} \mathrm{O}_{5} \mathrm{PS}_{2}$

\begin{tabular}{|c|c|c|c|c|c|}
\hline \multicolumn{2}{|c|}{$\begin{array}{l}\text { Satellite cluster merging } \\
\text { (SCM) }\end{array}$} & \multicolumn{2}{|c|}{$\begin{array}{l}\text { Rectangular mass window } \\
\text { (RMW) }\end{array}$} & \multicolumn{2}{|c|}{ Difference } \\
\hline Mass (Da) & Int (\%) & Mass (Da) & Int (\%) & Mass (mDa) & Int (\%) \\
\hline & & 1170.5069 & 10.57 & & \\
\hline \multirow[t]{2}{*}{1170.5076} & 21.43 & 1170.5078 & 30.44 & 0.22 & 9.01 \\
\hline & & 1172.5049 & 51.40 & & \\
\hline 1172.5053 & 57.16 & 1172.5058 & 59.22 & 0.48 & 2.06 \\
\hline \multirow[t]{3}{*}{1173.5087} & 18.67 & 1173.5082 & 16.69 & 0.51 & 1.97 \\
\hline & & 1173.5091 & 19.32 & & \\
\hline & & 1174.5019 & 35.57 & & \\
\hline \multirow[t]{3}{*}{1174.5029} & 100.00 & 1174.5028 & 100.00 & 0.13 & 0.00 \\
\hline & & 1174.5037 & 57.64 & & \\
\hline & & 1175.5052 & 12.21 & & \\
\hline \multirow[t]{2}{*}{1175.5063} & 32.59 & 1175.5062 & 32.52 & 0.14 & 0.07 \\
\hline & & 1175.5071 & 18.90 & & \\
\hline \multirow[t]{2}{*}{1176.4992} & 11.59 & 1176.4989 & 12.99 & 0.26 & 1.40 \\
\hline & & 1176.4998 & 69.08 & & \\
\hline \multirow[t]{3}{*}{1176.5006} & 98.48 & 1176.5008 & 97.29 & 0.15 & 1.19 \\
\hline & & 1176.5017 & 28.16 & & \\
\hline & & 1177.5032 & 22.48 & & \\
\hline \multirow[t]{2}{*}{1177.5040} & 32.15 & 1177.5041 & 31.72 & 0.15 & 0.43 \\
\hline & & 1178.4969 & 25.14 & & \\
\hline \multirow[t]{2}{*}{1178.4980} & 71.02 & 1178.4978 & 67.21 & 0.20 & 3.81 \\
\hline & & 1178.4987 & 47.38 & & \\
\hline \multirow[t]{3}{*}{1179.5013} & 23.15 & 1179.5011 & 21.91 & 0.20 & 1.25 \\
\hline & & 1179.5021 & 15.51 & & \\
\hline & & 1180.4948 & 24.22 & & \\
\hline 1180.4956 & 32.24 & 1180.4957 & 32.71 & 0.13 & 0.48 \\
\hline 1181.4990 & 10.53 & 1181.4991 & 10.69 & 0.12 & 0.16 \\
\hline 1182.4929 & 11.27 & 1182.4928 & 11.77 & 0.16 & 0.51 \\
\hline
\end{tabular}




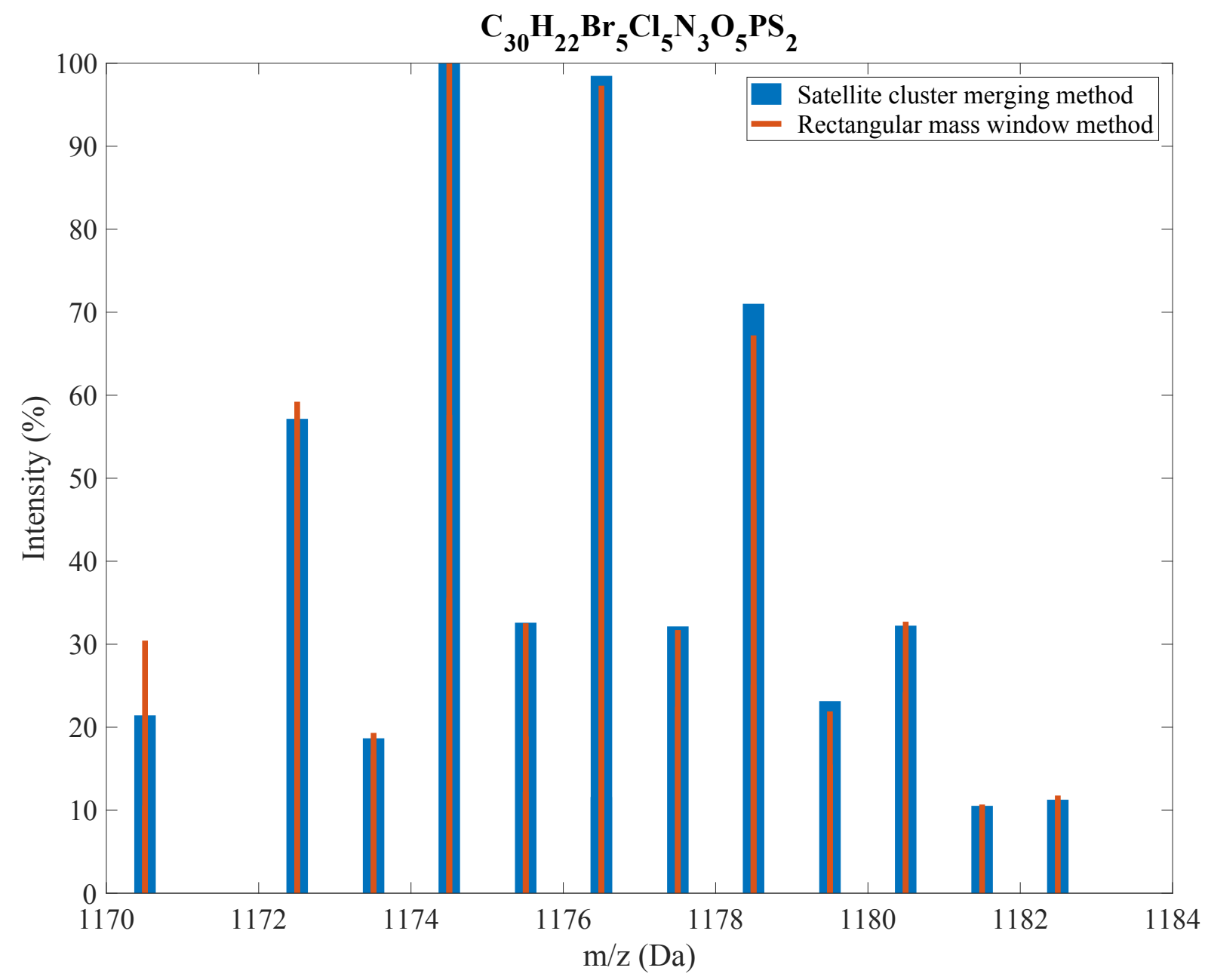

Figure S.2. Comparison between rectangular mass window (RMW) and satellite cluster merging (SCM) methods for $\mathrm{C}_{30} \mathrm{H}_{22} \mathrm{Br}_{5} \mathrm{Cl}_{5} \mathrm{~N}_{3} \mathrm{O}_{5} \mathrm{PS}_{2}$ isotopic distribution profile calculation. 
Table S.4. Isotope profile of $\mathrm{C}_{30} \mathrm{H}_{22} \mathrm{Br}_{5} \mathrm{Cl}_{5} \mathrm{~N}_{3} \mathrm{PO}_{5} \mathrm{~S}_{2}$

\begin{tabular}{|c|c|c|c|}
\hline Mass (Da) & $\begin{array}{c}\text { Profile intensity (\%) } \\
\text { considering all } \\
\text { elements share }\end{array}$ & $\begin{array}{c}\text { Profile intensity (\%) } \\
\text { regardless of impact } \\
\text { of H, N, P and O }\end{array}$ & $\begin{array}{c}\text { Absolute difference of } \\
\text { profile intensity (\%) } \\
\text { between two scenarios }\end{array}$ \\
\hline 1170.5076 & 21.43 & 21.43 & 0.00 \\
\hline 1172.5053 & 57.16 & 57.16 & 0.00 \\
\hline 1173.5087 & 18.67 & 18.55 & 0.12 \\
\hline 1174.5029 & 100.00 & 100.00 & 0.00 \\
\hline 1175.5063 & 32.59 & 32.45 & 0.15 \\
\hline 1176.4992 & 11.59 & 11.58 & 0.00 \\
\hline 1176.5006 & 98.48 & 98.48 & 0.19 \\
\hline 1177.5040 & 32.15 & 31.95 & 0.01 \\
\hline 1178.4980 & 71.02 & 71.01 & 0.11 \\
\hline 1179.5013 & 23.15 & 23.04 & 0.01 \\
\hline 1180.4956 & 32.24 & 32.23 & 0.07 \\
\hline 1181.4990 & 10.53 & 11.26 & 0.46 \\
\hline 1182.4929 & 11.27 & & \\
\hline
\end{tabular}




\section{S.4. Why the IPDC algorithm is computationally efficient for unknown compounds screening?}

In this work, 2,998,065 halogenated compounds that are potentially bioaccumulative, persistent and toxic (PBT) were targeted. The IPDC algorithm presents a computationally efficient method in compare to what a straightforward but inefficient method requires to produce similar results. The inefficient method requires calculating the isotopic model for each candidate separately, and then searching for isotopic models of candidate compounds in HRMS data similar to the approach of our previous publication ${ }^{3}$. The straightforward method is not feasible when a large number of compounds are targeted. In the straightforward method, if the calculation time of an isotopic model for a compound takes 0.01 seconds, 8 hours is required to only calculate the isotopic models for all compounds. If screening for the isotopic model of each compound in each chromatogram scans takes 0.0001 seconds, 832 hours is required to screen a GC-MS file for this number of compounds in the straightforward method. Therefore, the IPDC algorithm was designed to perform all these calculations in a computationally efficient way. Using the IPDC algorithm, generating isotopic pattern seeds (step 1), screening for isotopic patterns in HRMS data (step 2) and the chromatographic analysis (step 3) took $\sim 1$ minute, $\sim 5$ hours and $\sim 8$ hours, respectively. 


\section{S.5. Similar Seed Profile Combination Example}

The following $26 \mathrm{C} / \mathrm{Br} / \mathrm{Cl} / \mathrm{S}$ combinations result in similar isotope pattern seeds based on the conditions used in Table 2: $\left\{\mathrm{C}_{6} \mathrm{BrCl}_{7}\right\},\left\{\mathrm{C}_{6} \mathrm{BrCl}_{7} \mathrm{~S}\right\},\left\{\mathrm{C}_{6} \mathrm{BrCl}_{7} \mathrm{~S}_{2}\right\},\left\{\mathrm{C}_{6} \mathrm{Cl}_{9}\right\}$, $\left\{\mathrm{C}_{6} \mathrm{Cl}_{9} \mathrm{~S}\right\},\left\{\mathrm{C}_{7} \mathrm{BrCl}_{7}\right\},\left\{\mathrm{C}_{7} \mathrm{BrCl}_{7} \mathrm{~S}\right\},\left\{\mathrm{C}_{7} \mathrm{BrCl}_{7} \mathrm{~S}_{2}\right\},\left\{\mathrm{C}_{7} \mathrm{Cl}_{9}\right\},\left\{\mathrm{C}_{7} \mathrm{Cl}_{9} \mathrm{~S}\right\},\left\{\mathrm{C}_{8} \mathrm{BrCl}_{7}\right\},\left\{\mathrm{C}_{8} \mathrm{BrCl}_{7} \mathrm{~S}\right\}$, $\left\{\mathrm{C}_{8} \mathrm{BrCl}_{7} \mathrm{~S}_{2}\right\},\left\{\mathrm{C}_{8} \mathrm{Cl}_{9}\right\},\left\{\mathrm{C}_{8} \mathrm{Cl}_{9} \mathrm{~S}\right\},\left\{\mathrm{C}_{9} \mathrm{BrCl}_{7}\right\},\left\{\mathrm{C}_{9} \mathrm{BrCl}_{7} \mathrm{~S}\right\},\left\{\mathrm{C}_{9} \mathrm{BrCl}_{7} \mathrm{~S}_{2}\right\},\left\{\mathrm{C}_{9} \mathrm{Cl}_{9}\right\},\left\{\mathrm{C}_{9} \mathrm{Cl}_{9} \mathrm{~S}\right\}$, $\left\{\mathrm{C}_{10} \mathrm{BrCl}_{7} \mathrm{~S}\right\},\left\{\mathrm{C}_{10} \mathrm{BrCl}_{7} \mathrm{~S}_{2}\right\},\left\{\mathrm{C}_{10} \mathrm{Cl}{ }_{9} \mathrm{~S}\right\},\left\{\mathrm{C}_{11} \mathrm{BrCl}_{7} \mathrm{~S}\right\},\left\{\mathrm{C}_{12} \mathrm{BrCl}_{7} \mathrm{~S}\right\}$ and $\left\{\mathrm{C}_{10} \mathrm{Cl}_{9}\right\}$.

Table S.5. $\left\{\mathrm{C}_{10} \mathrm{Cl}_{9}\right\}$ seed profile comparison with average seed profile

\begin{tabular}{|c|c|c|c|c|c|c|}
\hline \multirow[b]{2}{*}{$\begin{array}{c}\text { Isotopologue } \\
\text { number }\end{array}$} & \multicolumn{2}{|c|}{$\begin{array}{l}\text { The average seed profile } \\
\text { among all these combinations }\end{array}$} & \multicolumn{2}{|c|}{$\mathrm{C}_{10} \mathrm{Cl}_{9}$} seed profile & \multicolumn{2}{|c|}{ Difference } \\
\hline & $\begin{array}{l}\text { Mass difference } \\
\text { from the most } \\
\text { abundant } \\
\text { isotopologue } \\
\text { (Da) }\end{array}$ & $\begin{array}{c}\text { Intensity } \\
\text { profile }(\%)\end{array}$ & $\begin{array}{c}\text { Mass } \\
\text { difference } \\
\text { from the most } \\
\text { abundant } \\
\text { isotopologue } \\
\text { (Da) }\end{array}$ & $\begin{array}{c}\text { Intensity } \\
\text { profile }(\%)\end{array}$ & $\begin{array}{c}\text { Mass } \\
\text { difference } \\
(\mathrm{mDa})\end{array}$ & $\begin{array}{l}\text { Intensity } \\
\text { profile } \\
\text { difference } \\
(\%)\end{array}$ \\
\hline 1 & -3.9943 & 24.37 & -3.9941 & 27.19 & 0.28 & 2.82 \\
\hline 2 & -1.9971 & 75.69 & -1.9970 & 78.22 & 0.12 & 2.53 \\
\hline 3 & 0 & 100 & 0 & 100 & 0.00 & 0 \\
\hline 4 & 1.0033 & 8.86 & 1.0033 & 10.82 & 0.00 & 1.96 \\
\hline 5 & 1.9971 & 76.02 & 1.9970 & 74.58 & 0.08 & 1.44 \\
\hline 6 & 3.9942 & 36.39 & 3.9941 & 35.75 & 0.13 & 0.64 \\
\hline 7 & 5.9913 & 11.39 & 5.9911 & 11.43 & 0.17 & 0.04 \\
\hline
\end{tabular}




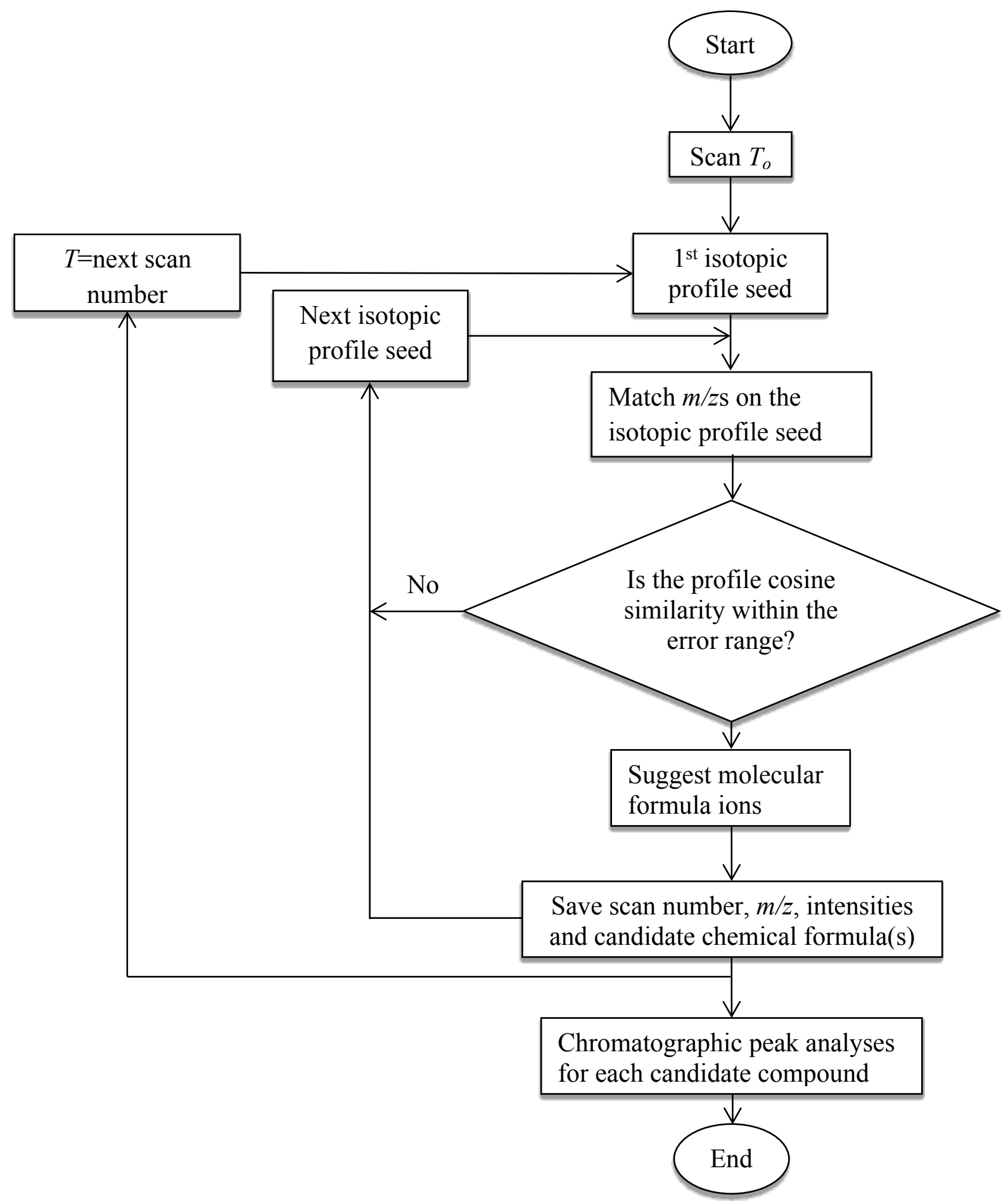

Figure S.3. Flowchart of the IPDC algorithm 
Table S.6. The criteria for the IPDC algorithm in positive and negative modes.

\begin{tabular}{|c|c|c|}
\hline & Algorithm step & Criteria \\
\hline $\mathbf{a}$ & $\begin{array}{l}\text { Isotope pattern seeds } \\
\text { calculation }{ }^{1}\end{array}$ & $\begin{array}{l}\text { - Neighboring isotopologue space }<1 \mathrm{mDa} \\
\text { - Theoretical intensity threshold }<\text { minimum }\{10 \% \text {, percentage equal to number } \\
\text { of carbon atoms }\}\end{array}$ \\
\hline b & $\begin{array}{l}\text { Combining similar } \\
\text { isotope patterns }\end{array}$ & $\begin{array}{l}\text { - The same number of isotopologues in all isotope pattern seeds } \\
\text { - Maximum mass difference between corresponding isotopologues }<1 \mathrm{mDa} \\
\text { - Profile cosine similarity }>999 \%\end{array}$ \\
\hline c & $m / z$ screening & $\begin{array}{l}\text { - Mass error to detect }[\mathrm{M}],[\mathrm{M}+1], \ldots \text { isotopologues from MAIso mass }<15 \mathrm{mDa} \\
\text { - Profile cosine similarity }>950 \% 0 \\
\text { - Intensity count of the MAIso mass }>500\end{array}$ \\
\hline d & $\begin{array}{l}\text { Molecular formula } \\
\text { assignment }\end{array}$ & $\begin{array}{l}\text { - Mass error of the MAIso mass }<10 \mathrm{mDa} \\
\text { - Normalized Euclidean mass error }<10 \mathrm{mDa} \\
\text { - Profile cosine similarity }>950 \%\end{array}$ \\
\hline e & $\begin{array}{l}\text { Chromatographic peak } \\
\text { smoothing }\end{array}$ & $\begin{array}{l}\text { - Maximum space between two matched chromatogram scans to fill missing } \\
\text { chromatogram scans }=10 \text { scans }^{2} \\
\text { - Smoothing window }=10 \text { scans }^{2}\end{array}$ \\
\hline f & $\begin{array}{l}\text { Chromatographic peak } \\
\text { detection }\end{array}$ & $\begin{array}{l}\text { - Maximum difference between two neighboring local minima on the } \\
\text { chromatogram curve }=10 \text { scans }^{2} \\
\text { - Low abundant isomers must have above }\left\{\begin{array}{r}1 \%, N>3 \\
10 \%, N \leq 3\end{array} \text { of the intensity height }\right. \\
\text { of the most abundant isomer }{ }^{3} \\
\text { - Maximum number of isomers for each compound }=\text { The most }\left\{\begin{array}{r}25, N>3 \\
5, N \leq 3\end{array}\right. \\
\text { abundant isomers } \\
3\end{array}$ \\
\hline $\mathbf{g}$ & $\begin{array}{l}\text { Chromatographic peak } \\
\text { evaluation }\end{array}$ & $\begin{array}{l}N D C S \geq 3 \\
R C S \geq 25 \% \\
R P W \leq 0.8\end{array}$ \\
\hline
\end{tabular}

${ }^{1}$ See section S.3

2 The difference between two sequential scans is 0.288 seconds in low energy channel in this work

${ }^{3}$ A multicriteria condition with respect to $N$ is used to remove random peaks with lower number of isotopologues 


\section{S.6. Similar Isotope Profiles Example}

Although matching the isotope profile is the main core of the IPDC algorithm, the isotopic distribution profile is not a unique signature and many compounds may partially or completely share similar isotopic profiles. For example, the isotopic profile of $\left[\mathrm{C}_{12} \mathrm{H}_{5} \mathrm{Br}_{2} \mathrm{O}_{2}\right]^{-}$, which may represent the $[\mathrm{M}-\mathrm{Br}+\mathrm{O}]^{-}$ions for tribromodibenzofuran has an isotopic profile that is in part similar to $\left[\mathrm{C}_{12} \mathrm{H}_{4} \mathrm{Cl}_{5} \mathrm{O}\right]^{-}$, which may be indicative of the $[\mathrm{M}-\mathrm{Cl}+\mathrm{O}]^{-}$ion of hexachlorobiphenyl (Figure S.4). In this case, even the extracted ion chromatograms of these two ions are very similar (Figure S.5). The scoring function provides a higher score for the true positive identifications in respect to false positive identifications as shown in Table S.7.

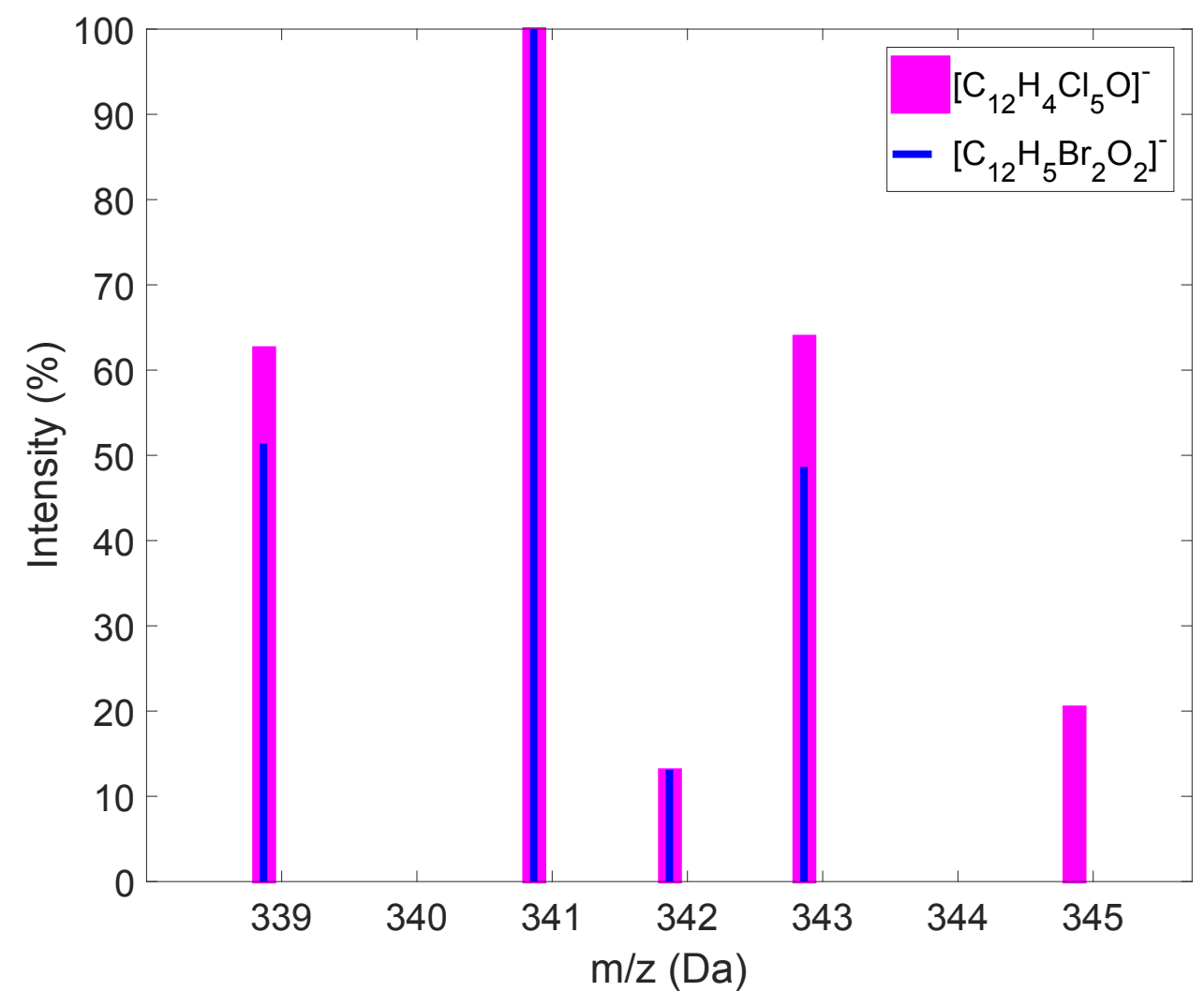

Figure S.4. Comparison of isotope profile of $\left[\mathrm{C}_{12} \mathrm{H}_{5} \mathrm{Br}_{2} \mathrm{O}_{2}\right]^{-}$and $\left[\mathrm{C}_{12} \mathrm{H}_{4} \mathrm{Cl}_{5} \mathrm{O}\right]^{-}$ions 

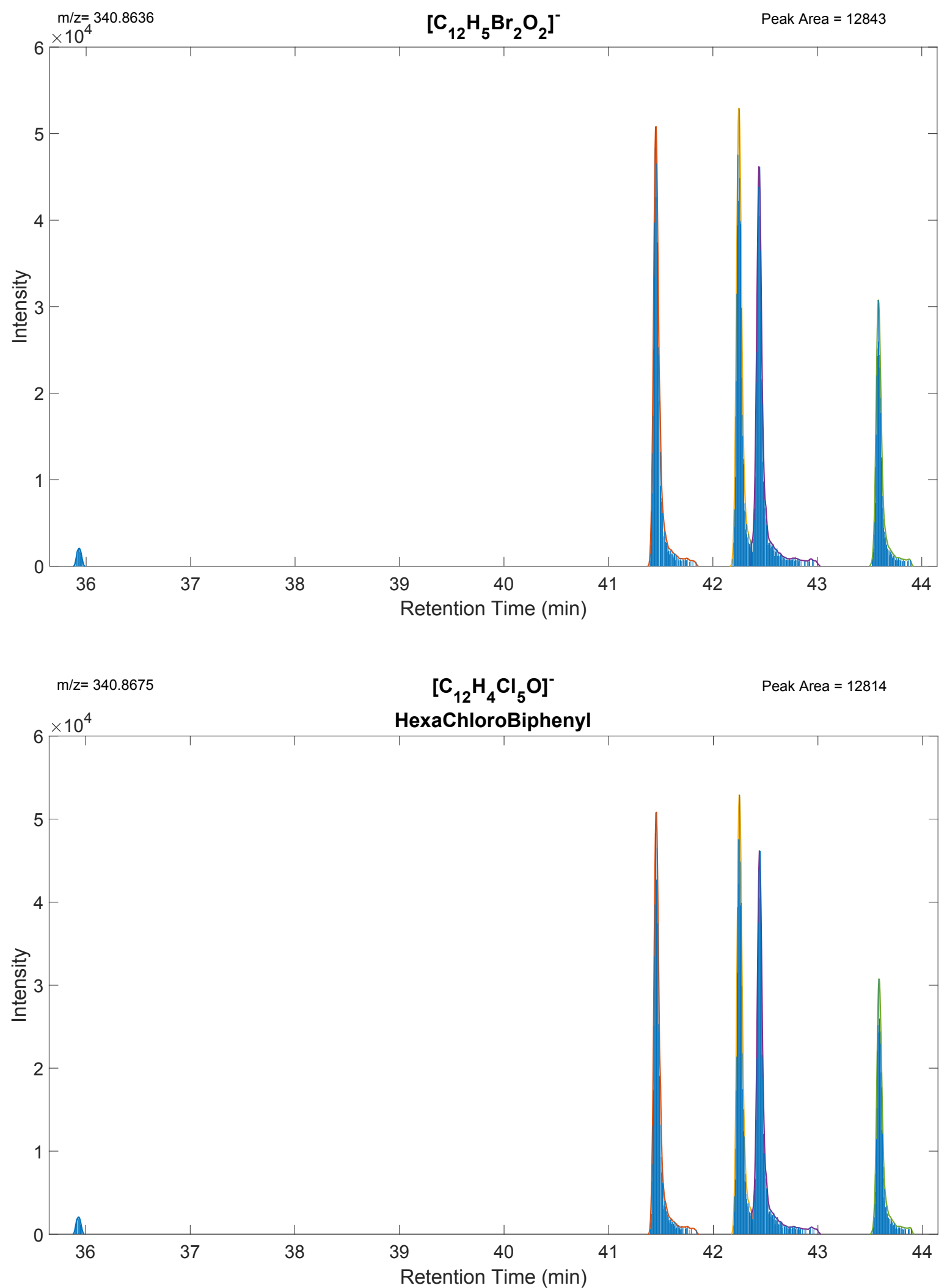

Figure S.5. Similarity of chromatogram traces of $\left[\mathrm{C}_{12} \mathrm{H}_{5} \mathrm{Br}_{2} \mathrm{O}_{2}\right]^{-}$and $\left[\mathrm{C}_{12} \mathrm{H}_{4} \mathrm{Cl}_{5} \mathrm{O}\right]^{-}$ions in PCB standard. 
Table S.7. Comparison of identification of $\left[\mathrm{C}_{12} \mathrm{H}_{4} \mathrm{Cl}_{5} \mathrm{O}\right]^{-}$and $\left[\mathrm{C}_{12} \mathrm{H}_{5} \mathrm{Br}_{2} \mathrm{O}_{2}\right]^{-}$ions using the IPDC algorithm.

\begin{tabular}{|c|c|c|c|c|c|c|c|c|c|c|c|c|}
\hline $\begin{array}{l}\text { Molecular } \\
\text { formula }\end{array}$ & $\begin{array}{l}\text { Isomer } \\
\text { number }\end{array}$ & $\begin{array}{l}\text { Detected } \\
\text { mass (Da) }\end{array}$ & $\begin{array}{c}\text { Exact } \\
\text { mass of } \\
\text { molecular } \\
\text { ion }(\mathrm{Da})\end{array}$ & Intensity & $\begin{array}{l}\overline{N E M E} \\
(\mathrm{mDa})\end{array}$ & $\begin{array}{l}\overline{P C S} \\
(\% \mathrm{o})\end{array}$ & $\begin{array}{l}\text { Retention } \\
\text { time } \\
\text { (min) }\end{array}$ & $\begin{array}{l}\text { Peak } \\
\text { Area }\end{array}$ & $N D C S$ & $\begin{array}{l}R C S \\
(\%)\end{array}$ & $R P W$ & $\begin{array}{c}\text { Identification } \\
\text { Score }\end{array}$ \\
\hline \multirow{6}{*}[\mathrm{C}_{12}\mathrm{H}_{4}\mathrm{Cl}_{5}\mathrm{O}]{$^{-}$} & 155 & 340.8630 & 340.8675 & 2056 & 4.99 & 999.06 & 35.936 & 112 & 13 & 87 & 0.49 & $2.32 \mathrm{E}+03$ \\
\hline & 167 & 340.8676 & 340.8675 & 50807 & 0.16 & 999.85 & 41.452 & 3535 & 71 & 78 & 0.11 & $1.34 \mathrm{E}+05$ \\
\hline & 156 & 340.8694 & 340.8675 & 52916 & 1.76 & 999.96 & 42.253 & 3457 & 36 & 92 & 0.28 & $5.13 \mathrm{E}+04$ \\
\hline & 157 & 340.8700 & 340.8675 & 46171 & 1.30 & 999.99 & 42.450 & 3449 & 93 & 83 & 0.10 & $1.35 \mathrm{E}+05$ \\
\hline & 169 & 340.8668 & 340.8675 & 30747 & 0.69 & 999.98 & 43.584 & 2196 & 67 & 86 & 0.11 & $9.88 \mathrm{E}+04$ \\
\hline & & & & & & & & 12749 & 280 & & & \\
\hline \multirow{6}{*}[\mathrm{C}_{12}\mathrm{H}_{5}\mathrm{Br}_{2}\mathrm{O}_{2}]{$^{-}$} & 1 & 340.8630 & 340.8636 & 2056 & 1.49 & 994.30 & 35.936 & 112 & 13 & 87 & 0.49 & $1.20 \mathrm{E}+03$ \\
\hline & 2 & 340.8676 & 340.8636 & 50807 & 4.02 & 992.77 & 41.452 & 3549 & 68 & 75 & 0.11 & $4.31 \mathrm{E}+04$ \\
\hline & 3 & 340.8694 & 340.8636 & 52916 & 5.68 & 993.81 & 42.253 & 3465 & 36 & 91 & 0.27 & $2.12 \mathrm{E}+04$ \\
\hline & 4 & 340.8700 & 340.8636 & 46171 & 5.23 & 993.26 & 42.450 & 3455 & 94 & 84 & 0.10 & $5.38 \mathrm{E}+04$ \\
\hline & 5 & 340.8668 & 340.8636 & 30747 & 3.22 & 993.21 & 43.584 & 2197 & 67 & 86 & 0.11 & $3.83 \mathrm{E}+04$ \\
\hline & & & & & & & & 12777 & 278 & & & \\
\hline
\end{tabular}




\section{S.8. Search Criteria Evaluation}

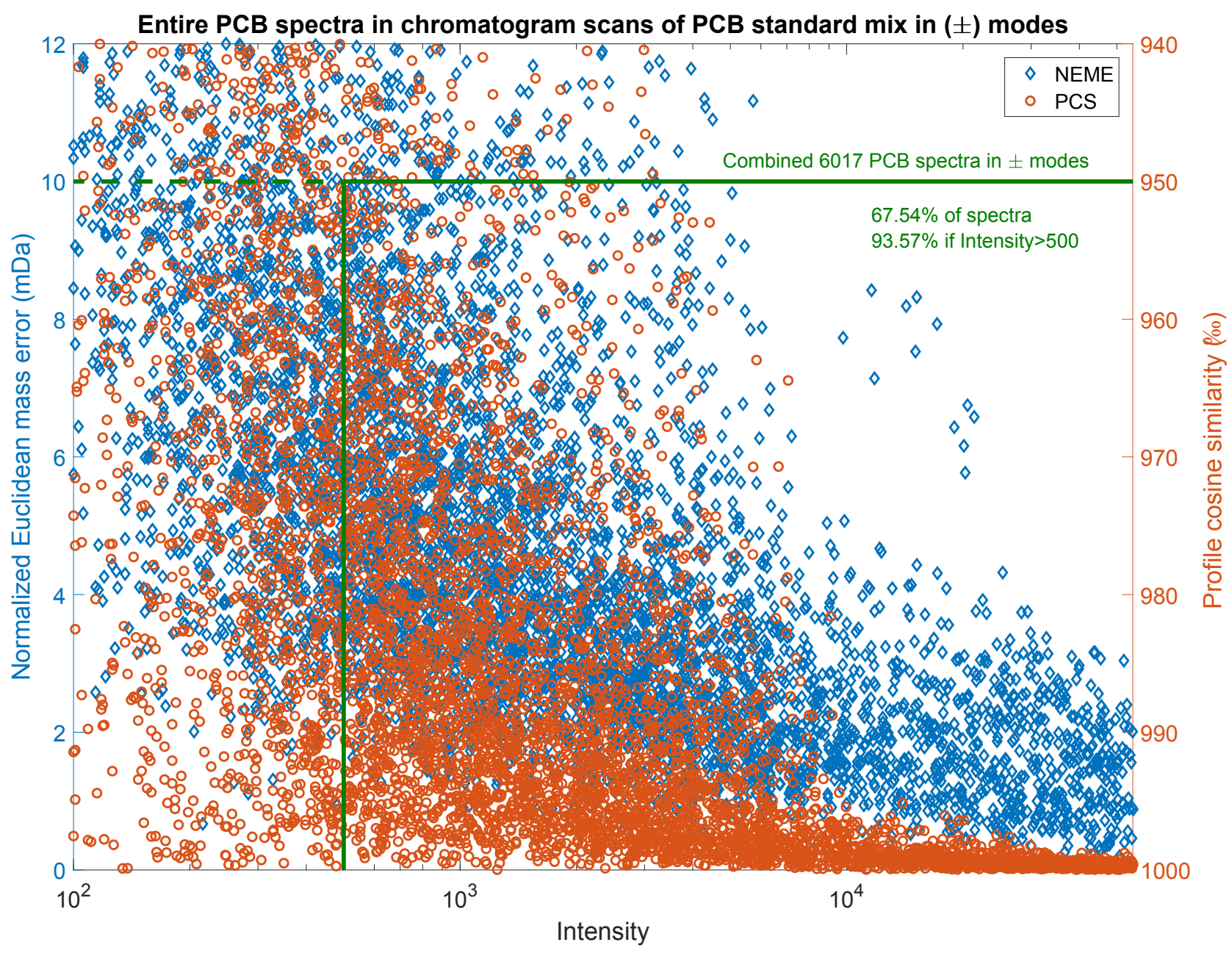

Figure S.6. Evaluation of search parameters used in Table 2 using PCB reference standards. Each point represents a chromatogram scan that contains PCB mass spectral peaks. 
Mass error of the most abundant isotopologue for

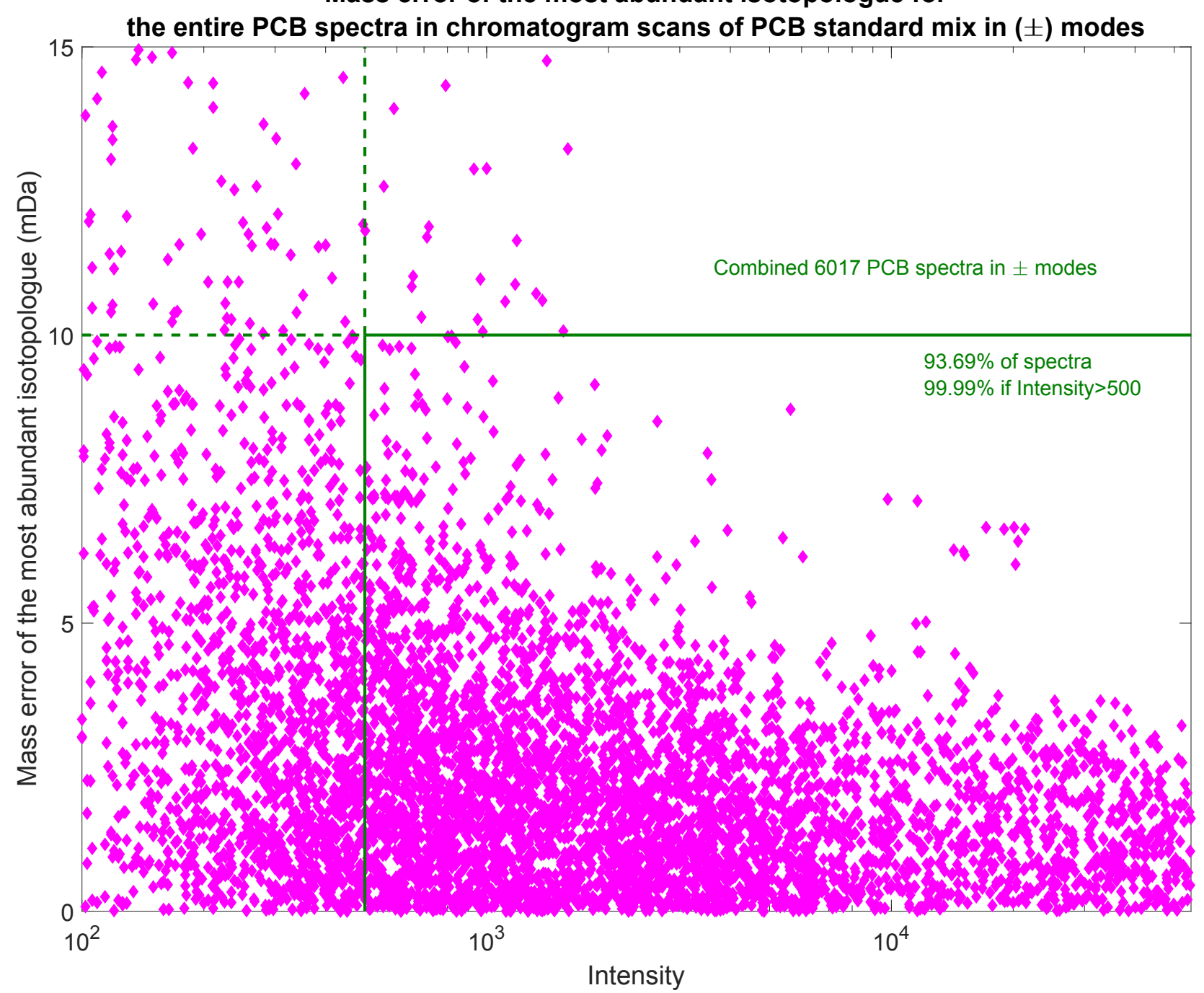

Figure S.7. Evaluation of mass error for the most abundant isotopologue used in molecular formulas assignment using PCB reference standards. Each point represents a chromatogram scan that contains PCB mass spectral peaks. 


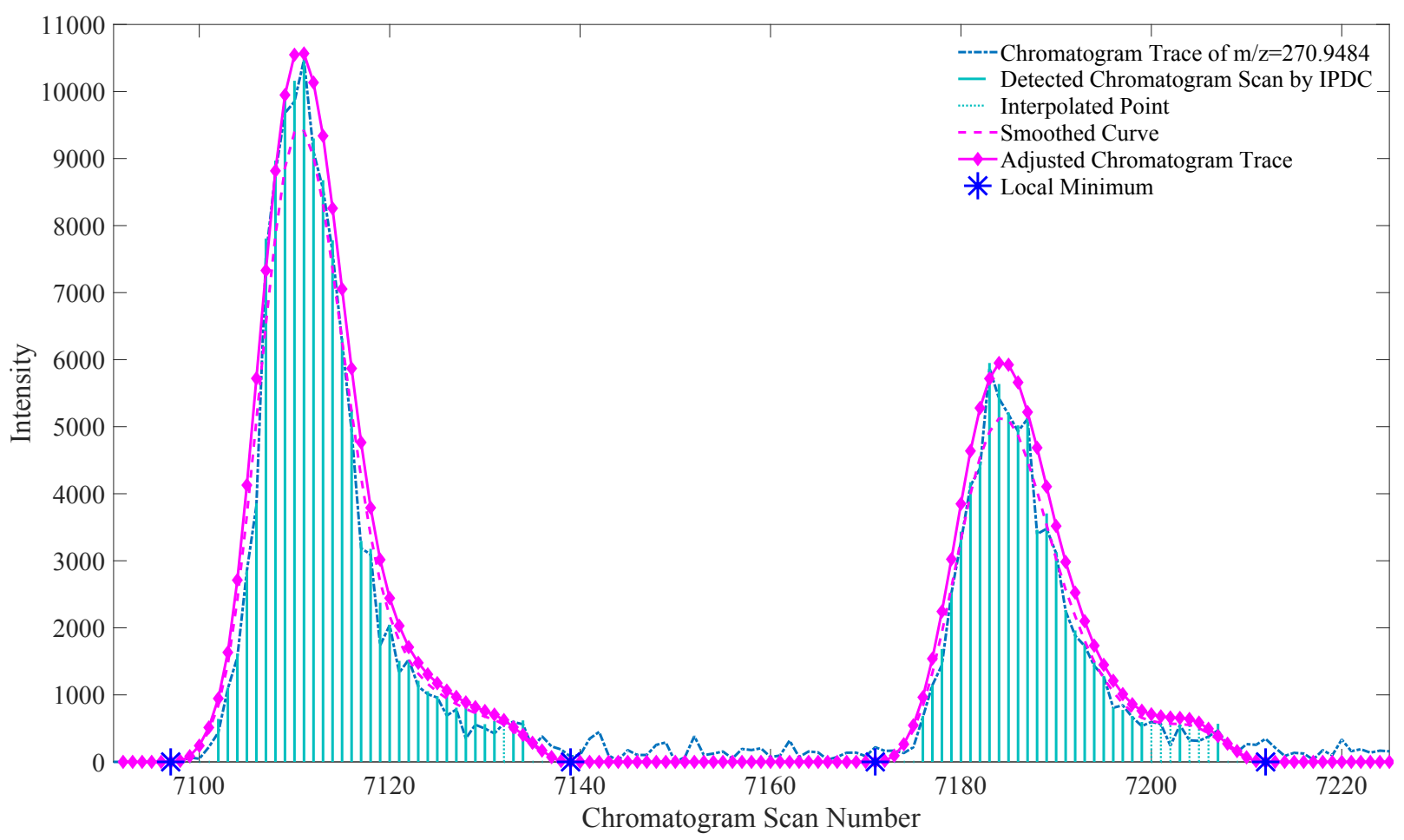

(a)

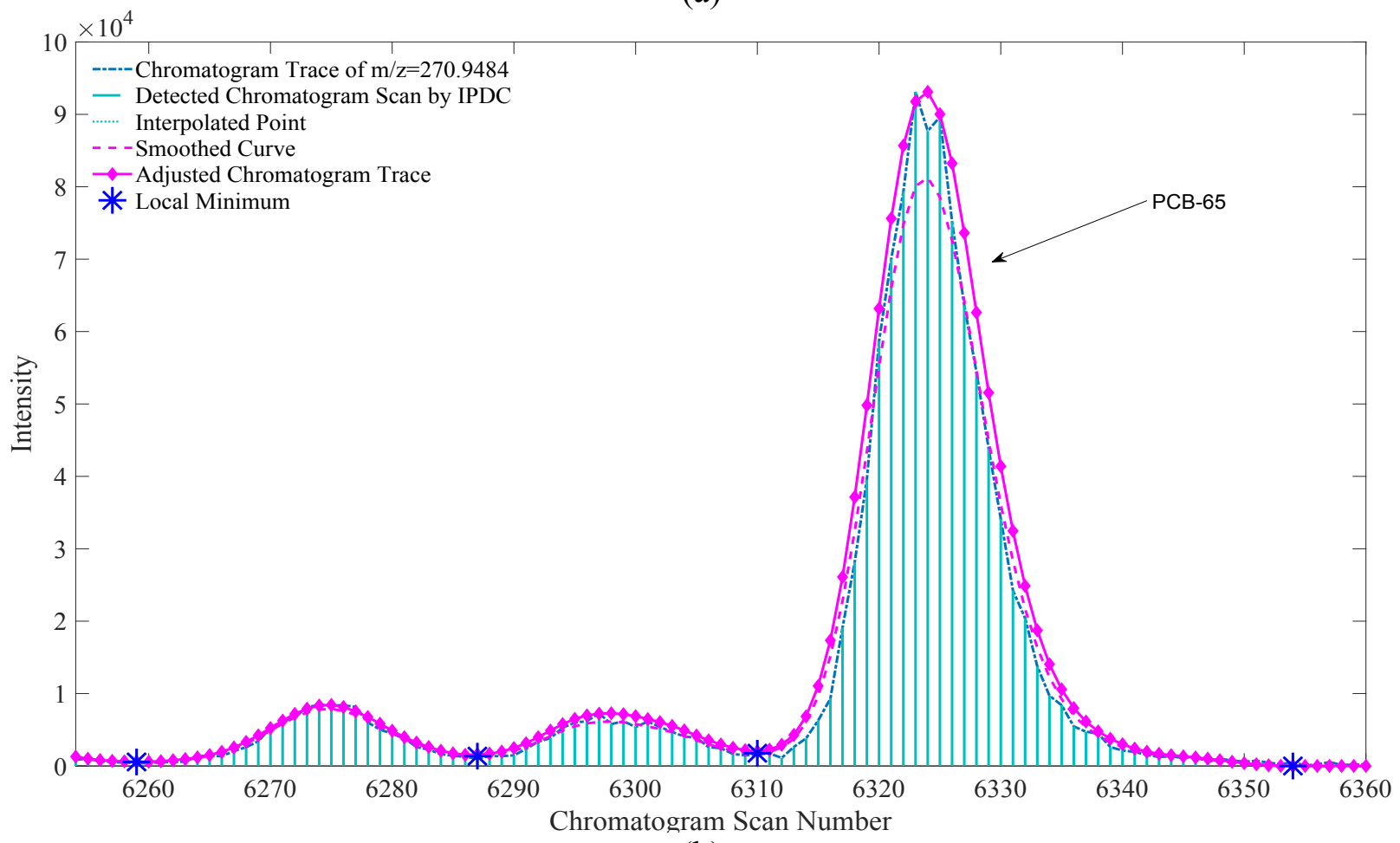

(b)

Figure S.8. Chromatographic peak analyses from IPDC algorithm results for a) PCB-81 and 77 in PCBs standard presented as $\left[\mathrm{C}_{12} \mathrm{H}_{6} \mathrm{Cl}_{3} \mathrm{O}\right]^{-}$ion, b) PCB-65 in LMLT 2016 presented as $\left[\mathrm{C}_{12} \mathrm{H}_{6} \mathrm{Cl}_{3} \mathrm{O}\right]^{-}$ion. 


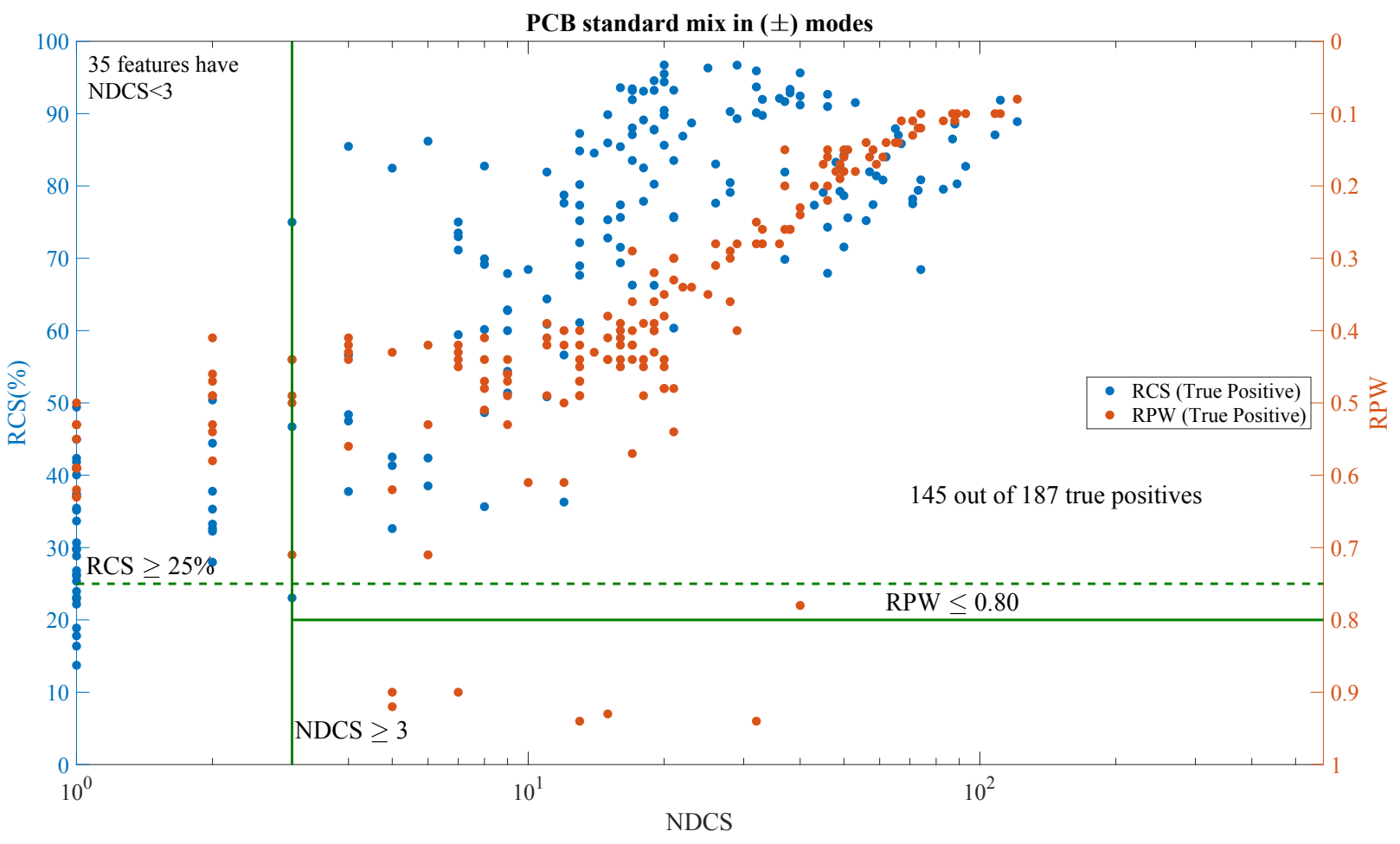

(a)

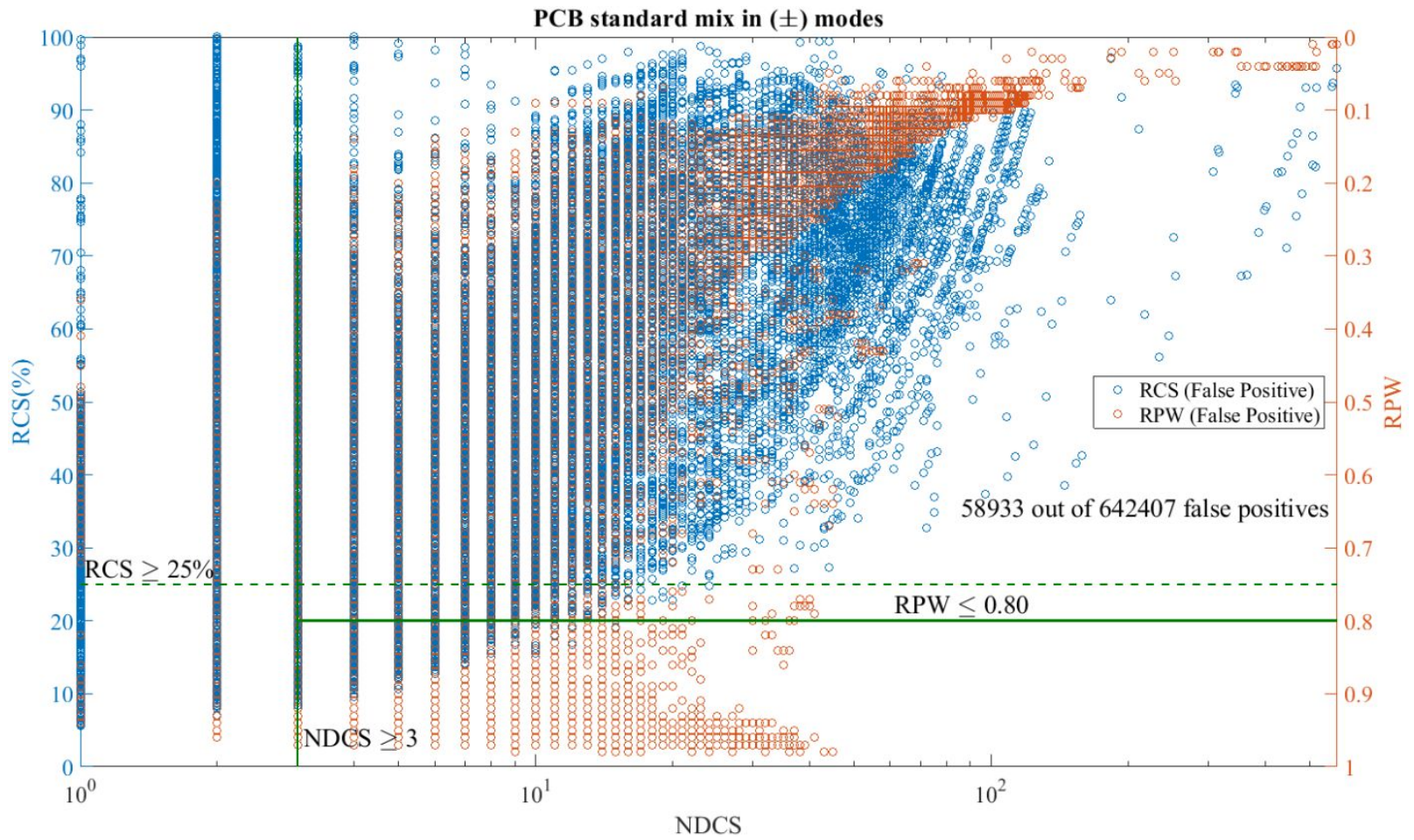

(b)

Figure S.9. False positive and true positive identifications analysis for four PCB standard mix 
injections. (a) True positive identifications, and (b) False positive identifications.

\section{S.9. Study the effect of weight of each parameter in the scoring formula}

The score function used in this study is calculated using the following equation:

$$
S=\frac{N^{a}\left(\log _{10}\left(\frac{\text { Int }}{500}\right)\right)\left(\log _{10}\left(\frac{P A}{13.92}\right)\right)\left(\frac{\overline{P C S}}{1000 \% 0}\right)^{b} N D C S^{c} R C S^{d}}{\left(\frac{\overline{N E M E}}{10 m D a}\right)^{e} R P W^{f}}
$$

where, Int and $P A$ represent the intensity and peak area of a feature, respectively and were scaled to their minimum expected values based on the search criteria. To correlate the coefficients $a-f$ of parameters in equation (S.1), the following objective function was minimized in the triplicate PCB standard injection data using the genetic algorithm.

$$
\begin{gathered}
O F=\left|1-\frac{\sum_{i=1}^{n_{i}} I_{1}\left(S_{i}\right)}{n_{i}}\right| \\
I_{1}\left(S_{i}\right)=\left\{\begin{array}{l}
1, S_{i} \in\left(\text { Top } k^{\text {th }}\right. \text { tentative molecular formulas) } \\
0, S_{i} \notin \text { (Top } k^{\text {th }} \text { tentative molecular formulas) }
\end{array}\right.
\end{gathered}
$$

where $S_{i}$ and $n_{i}$ demonstrate score of PCB features and number of PCB features, respectively. This objective function improves the ranking of the tentative molecular formulas by putting PCB molecular formulas among top $k$ ( $k=10$ in this work) tentative molecular formulas. The optimal coefficients $a-f$ are presented in Table S.8. It was found that $N$ has the highest impact on the overall score.

In order to understand the impact of weight of parameters $(f(X))$ in equation (S.1), the percentage of increase in the overall score is calculated by evaluating small incremental increase on two extremes and a middle point value of each parameter and results are shown in Table S.8. The following equation is used to calculate the percentage of the score increase. 


$$
\text { percentage of increase }=\frac{S_{X}+\text { increment }-S_{X}}{S_{X}} * 100 \%=\left(\frac{f(X+\text { increment })}{f(X)}-1\right) * 100 \%
$$

Table S.8. Study weight of each parameter on overall score.

\begin{tabular}{|c|c|c|c|}
\hline & $\begin{array}{l}\text { Coefficient } \\
\text { in Eq (3) }\end{array}$ & $\begin{array}{l}\text { Increment } \\
\text { size }\end{array}$ & $\begin{array}{l}\text { Percentage of score increase by increment size of } \\
\text { each variable }(\%)\end{array}$ \\
\hline$N$ & 2.84 & 1 & $\begin{array}{l}+126 \% \text { maximum increase from } 3 \rightarrow 4 \text { isotopologues } \\
+46 \% \text { from } 7 \rightarrow 8 \text { isotopologues } \\
+21 \% \text { minimum increase from } 14 \rightarrow 15 \text { isotopologues }\end{array}$ \\
\hline Int & - & 100 & $\begin{array}{l}+39.68 \% \text { maximum increase at intensity of } 700 \rightarrow 800 \\
+1.92 \% \text { at intensity of } 3000 \rightarrow 3100 \\
+0.82 \% \text { at intensity } 5000 \rightarrow 5100\end{array}$ \\
\hline $\boldsymbol{P A}$ & - & 10 & $\begin{array}{l}+112.10 \% \text { maximum increase at area of } 34 \text { (Three } \\
\text { consecutive chromatogram scans with intensity of } 700 \text { ) } \\
+2.28 \% \text { at area from } 170 \rightarrow 180 \\
+1.43 \% \text { at peak area from } 240 \rightarrow 250\end{array}$ \\
\hline$\overline{P C S}$ & 27.09 & $1 \%$ & $\begin{array}{l}+2.89 \% \text { maximum increase from } 950 \% \rightarrow 951 \% \\
+2.74 \% \text { minimum increase from } 999 \% \rightarrow 1000 \%\end{array}$ \\
\hline$N C S$ & 0.46 & 1 & $\begin{array}{l}+21 \% \text { maximum increase from } 2 \rightarrow 3 \text { scans } \\
+5.07 \% \text { from } 9 \rightarrow 10 \text { scans (Tentative candidates) }\end{array}$ \\
\hline$R C S$ & 0.47 & $1 \%$ & $\begin{array}{l}+16.98 \% \text { from } 25 \% \rightarrow 26 \% \text { (Tentative candidates) } \\
+5.92 \% \text { from } 69 \% \rightarrow 70 \% \text { (Intense peaks) }\end{array}$ \\
\hline$\overline{N E M E}$ & 0.08 & $1 \mathrm{mDa}$ & $\begin{array}{l}-53 \% \text { maximum decrease from } 1 \mathrm{mDa} \rightarrow 2 \mathrm{mDa} \\
-8.4 \% \text { minimum decrease from } 9 \mathrm{mDa} \rightarrow 10 \mathrm{mDa}\end{array}$ \\
\hline$R P W$ & 0.57 & 0.01 & $\begin{array}{l}-18.5 \% \text { from } 0.30 \rightarrow 0.31 \text { (High abundant peaks like PCBs) } \\
-10.4 \% \text { from } 0.54 \rightarrow 0.55 \text { (Ideal value) } \\
-7.14 \% \text { from } 0.79 \rightarrow 0.80 \text { (False positive) }\end{array}$ \\
\hline
\end{tabular}




\section{S.10. Empirical equations for predicting boiling point of halogenated compounds}

Riazi and Daubert 4, 5 developed an equation to generate empirical equations for prediction of physical properties $(\theta)$ of undefined hydrocarbon mixtures.

$$
\theta=a \theta_{1}^{b} \theta_{2}^{c}
$$

where $\theta_{1}$ and $\theta_{2}$ represent molecular force and molecular size of the compound respectively; and $a-c$ are constants obtained by correlation. The following modification was made to develop an empirical equation to calculate the boiling point $\left(T_{b}\right)$ of halogenated compounds:

$$
T_{b}=a(m-35.453 \times c l-79.904 \times b r)^{b}+c \times c l^{d}+e \times b r^{f}-273.15
$$

where $m, c l$ and $b r$ are mass $(\mathrm{Da})$, the number chlorine and bromine atoms in the halogenated compound, respectively. This coefficient of this equation was obtained by correlating the PCBs and PBDEs boiling point (presented in Table S.9)

$$
T_{b}=52.5453(m-35.453 c l-79.904 b r)^{0.4711}+17.1596 c l^{1.0718}+80.5543 b r^{0.9491}-273.15
$$

Table S.10 presents the efficacy of equation (S.7) on predicting boiling points of some common halogenated contaminants in Great Lakes lake trout and compared to equation (S.7). Up to $\pm 20 \%$ error can be expected for the boiling point calculated by equation (S.7). 
Table S.9. Deviation of boiling point of PCBs and PBDEs from Equation (S.7)

\begin{tabular}{|c|c|c|c|c|c|c|}
\hline Name & $\begin{array}{l}\text { Molecular } \\
\text { formula }\end{array}$ & Mass & $\begin{array}{c}\text { Experimental } \\
\text { Boiling Point } \\
\qquad\left({ }^{\circ} \mathrm{C}\right)\end{array}$ & $\begin{array}{c}\text { Predicted } \\
\text { Boiling Point } \\
\left({ }^{\circ} \mathrm{C}\right) \text { by } \\
\text { Eq( S.3) }\end{array}$ & AAD (\%) & Reference \\
\hline \multirow{24}{*}{ PBDEs } & \multirow{3}{*}{$\mathrm{C}_{12} \mathrm{H}_{9} \mathrm{BrO}$} & \multirow{3}{*}{249.10} & 401 & 397 & 1.09 & \multirow{24}{*}[6]{} \\
\hline & & & 394 & 397 & 0.65 & \\
\hline & & & 395 & 397 & 0.45 & \\
\hline & \multirow{3}{*}{$\mathrm{C}_{12} \mathrm{H}_{8} \mathrm{Br}_{2} \mathrm{O}$} & \multirow{3}{*}{328.00} & 471 & 470 & 0.20 & \\
\hline & & & 467 & 470 & 0.73 & \\
\hline & & & 473 & 470 & 0.61 & \\
\hline & \multirow{2}{*}{$\mathrm{C}_{12} \mathrm{H}_{7} \mathrm{Br}_{3} \mathrm{O}$} & \multirow{2}{*}{406.90} & 543 & 542 & 0.19 & \\
\hline & & & 541 & 542 & 0.13 & \\
\hline & \multirow{5}{*}{$\mathrm{C}_{12} \mathrm{H}_{6} \mathrm{Br}_{4} \mathrm{O}$} & \multirow{5}{*}{485.79} & 613 & 612 & 0.28 & \\
\hline & & & 610 & 612 & 0.26 & \\
\hline & & & 607 & 612 & 0.72 & \\
\hline & & & 627 & 612 & 2.46 & \\
\hline & & & 626 & 612 & 2.33 & \\
\hline & \multirow{5}{*}{$\mathrm{C}_{12} \mathrm{H}_{5} \mathrm{Br}_{5} \mathrm{O}$} & \multirow{5}{*}{564.69} & 672 & 681 & 1.25 & \\
\hline & & & 682 & 681 & 0.21 & \\
\hline & & & 681 & 681 & 0.05 & \\
\hline & & & 678 & 681 & 0.44 & \\
\hline & & & 691 & 681 & 1.40 & \\
\hline & \multirow{6}{*}{$\mathrm{C}_{12} \mathrm{H}_{4} \mathrm{Br}_{6} \mathrm{O}$} & \multirow{6}{*}{643.58} & 730 & 749 & 2.69 & \\
\hline & & & 747 & 749 & 0.26 & \\
\hline & & & 736 & 749 & 1.78 & \\
\hline & & & 744 & 749 & 0.77 & \\
\hline & & & 742 & 749 & 0.94 & \\
\hline & & & 750 & 749 & 0.06 & \\
\hline \multirow{10}{*}{ PCBs } & $\mathrm{C}_{12} \mathrm{H}_{9} \mathrm{Cl}$ & 188.66 & 285 & 307 & 7.58 & \multirow{10}{*}{ [7] } \\
\hline & $\mathrm{C}_{12} \mathrm{H}_{8} \mathrm{Cl}_{2}$ & 223.10 & 312 & 324 & 3.77 & \\
\hline & $\mathrm{C}_{12} \mathrm{H}_{7} \mathrm{Cl}_{3}$ & 257.55 & 337 & 342 & 1.38 & \\
\hline & $\mathrm{C}_{12} \mathrm{H}_{6} \mathrm{Cl}_{4}$ & 291.99 & 360 & 360 & 0.00 & \\
\hline & $\mathrm{C}_{12} \mathrm{H}_{5} \mathrm{Cl}_{5}$ & 326.44 & 381 & 379 & 0.60 & \\
\hline & $\mathrm{C}_{12} \mathrm{H}_{4} \mathrm{Cl}_{6}$ & 360.88 & 400 & 398 & 0.57 & \\
\hline & $\mathrm{C}_{12} \mathrm{H}_{3} \mathrm{Cl}_{7}$ & 395.33 & 417 & 417 & 0.00 & \\
\hline & $\mathrm{C}_{12} \mathrm{H}_{2} \mathrm{Cl}_{8}$ & 429.77 & 432 & 436 & 1.03 & \\
\hline & $\mathrm{C}_{12} \mathrm{HCl}_{9}$ & 464.22 & 445 & 456 & 2.50 & \\
\hline & $\mathrm{C}_{12} \mathrm{Cl}_{10}$ & 498.66 & 456 & 476 & 4.37 & \\
\hline
\end{tabular}


Table S.10. Investigating Equation (6) on some halogenated compounds

\begin{tabular}{|l|c|c|c|c|c|c|}
\hline \multicolumn{1}{|c|}{ Name } & $\begin{array}{c}\text { Molecular } \\
\text { formula }\end{array}$ & Mass & $\begin{array}{c}\text { Boiling } \\
\text { Point from } \\
\text { different } \\
\text { sources }\left({ }^{\circ} \mathrm{C}\right)\end{array}$ & $\begin{array}{c}\text { Predicted } \\
\text { Boiling } \\
\text { Point }\left({ }^{\circ} \mathrm{C}\right) \\
\text { by Eq }(\mathrm{S} .7)\end{array}$ & $\begin{array}{c}\mathrm{AD} \\
(\%)\end{array}$ & Reference \\
\hline Dacthal & $\mathrm{C}_{10} \mathrm{H}_{6} \mathrm{Cl}_{4} \mathrm{O}_{4}$ & 331.95 & 343 & 417 & -21.57 & EPI Suite \\
\hline Perchloro styrene & $\mathrm{C}_{8} \mathrm{Cl}_{8}$ & 379.68 & 352 & 344 & -2.27 & EPI Suite \\
\hline Heptachlor epoxide & $\mathrm{C}_{10} \mathrm{H}_{5} \mathrm{Cl}_{7} \mathrm{O}$ & 389.3 & 340 & 404 & -18.82 & EPI Suite \\
\hline Hexachlorobenzene & $\mathrm{C}_{6} \mathrm{Cl}_{6}$ & 284.782 & 324 & 252 & 22.22 & SynQuest \\
\hline Perchloronaphthalene & $\mathrm{C}_{10} \mathrm{Cl}_{8}$ & 403.731 & 410 & 389 & 5.12 & NIOSH \\
\hline DDMU & $\mathrm{C}_{14} \mathrm{H}_{9} \mathrm{Cl}_{3}$ & 283.58 & 349 & 380 & -8.88 & EPI Suite \\
\hline Nonachlor & $\mathrm{C}_{10} \mathrm{H}_{5} \mathrm{Cl}_{9}$ & 444.224 & 372 & 418 & -12.36 & EPI Suite \\
\hline
\end{tabular}

${ }^{a}$ The references here are cross referenced from https://www.chemspider.com 


\section{S.11. Estimating the impact of boiling point on retention time}

Simpson et. al, ${ }^{6}$ showed that retention time of polybrominated diphenyl ethers have a linear correlation with their boiling point on DB-5 columns. Hence, the relationship between boiling points of candidate halogenated compounds and their retention time can be estimated to filter out features that are outside of the acceptable retention time range. Equation (S.7) presented in section S.10 was used to predict the boiling points of halogenated compounds.

The maximum retention time difference between two PCB isomers is around 8 minutes. Hence, to find the retention time boundaries versus the predicted boiling point, an 8 minute window based on the eluting pattern shown in Figure S.10 was used to develop the following boundaries.

Top boundary

$$
\frac{[R T(\text { early eluting HexaBDE })-8]-[6-8]}{B P(\text { early eluting HexaBDE })-G C \operatorname{temp}(@ 6 \mathrm{~min})}=\frac{x-[6-8]}{y-65}
$$

Bottom boundary

$$
\frac{[R T \text { (late eluting PCB })+8]-[6+8]}{B P(\text { late eluting PCB })-G C \text { temp }(@ 6 \mathrm{~min})}=\frac{x-[6+8]}{y-65}
$$

The retention time of the candidate compounds should be within the following boundaries.

$$
\begin{array}{cl}
\text { Top RT boundary } & R T_{\text {top }}=\left(0.8 \times T_{b^{-}}-96.1880\right) / 15.5940 \\
\text { Bottom RT boundary } & R T_{\text {Bottom }}=\left(1.2 \times T_{b}+64.5008\right) / 9.25
\end{array}
$$

The 0.8 and 1.2 coefficients in equations (S.8) and (S.9), respectively, were used to take into account associated errors with $T_{b}$ predictions. 


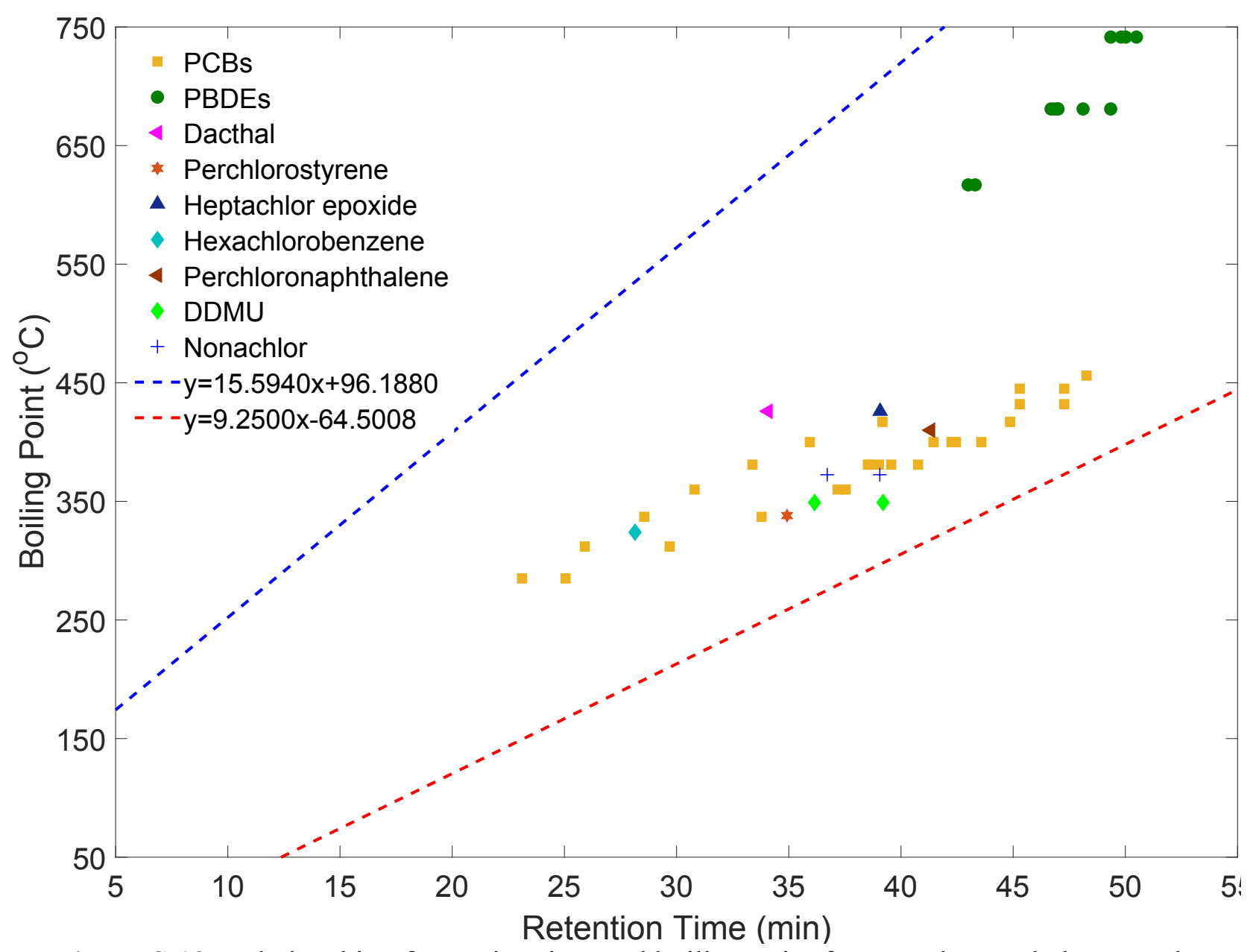

Figure S.10. Relationship of retention time and boiling point for some known halogenated compounds in Great Lakes Lake Trout. 


\section{S.12. Column Bleed Filter}

In some cases, column bleed masses are misidentified as the halogenated compounds. A mass defect for methyl siloxane $\left(\mathrm{Si}\left(\mathrm{CH}_{3}\right) \mathrm{O}\right)$ was used to remove features that are likely to be column bleed. The mass defect was calculated using equation (S.10).

$$
\begin{gathered}
\text { Mass defect }=\text { Exact mass } \times \operatorname{Coefficient}\left(\mathrm{Si}\left(\mathrm{CH}_{3}\right) \mathrm{O}\right)-\text { round down }(\text { Exact mass })+1 \\
\text { Coefficient }\left(\mathrm{Si}\left(\mathrm{CH}_{3}\right) \mathrm{O}\right)=59 / 58.99533
\end{gathered}
$$

A value of one was added to all mass defects to circumvent the wraparound effect ${ }^{8}$ and only a mass defect range between 0.5-1.25 was considered. A schematic of the mass defect plot for halogenated compounds is shown in Figure S.11. Halogenated features that have mass defects between 1.08-1.25 and masses less than $250 \mathrm{Da}$ (the blue box shown in Figure S.11) were removed.

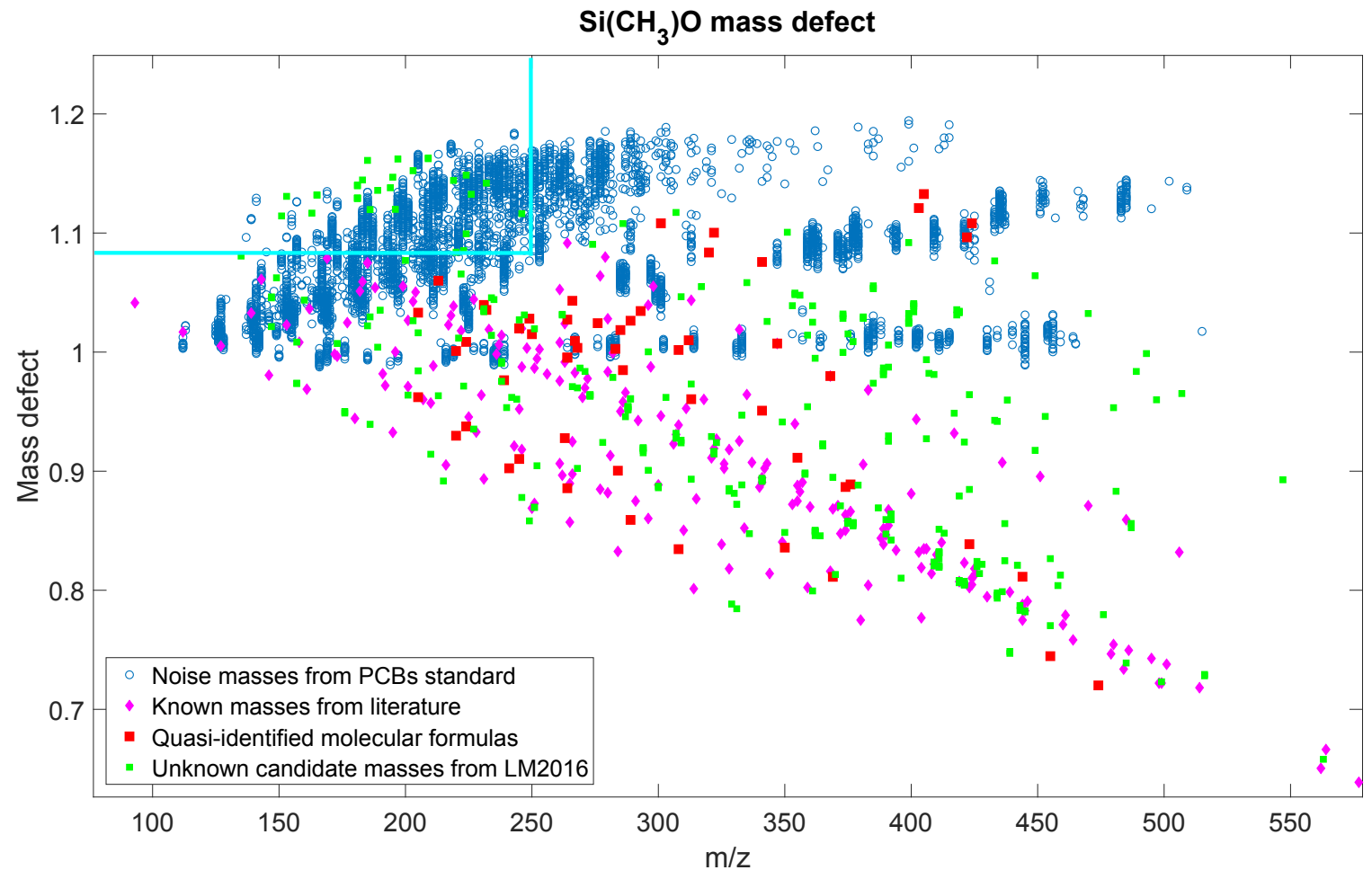

Figure S.11. Mass defect plot for halogenated features 


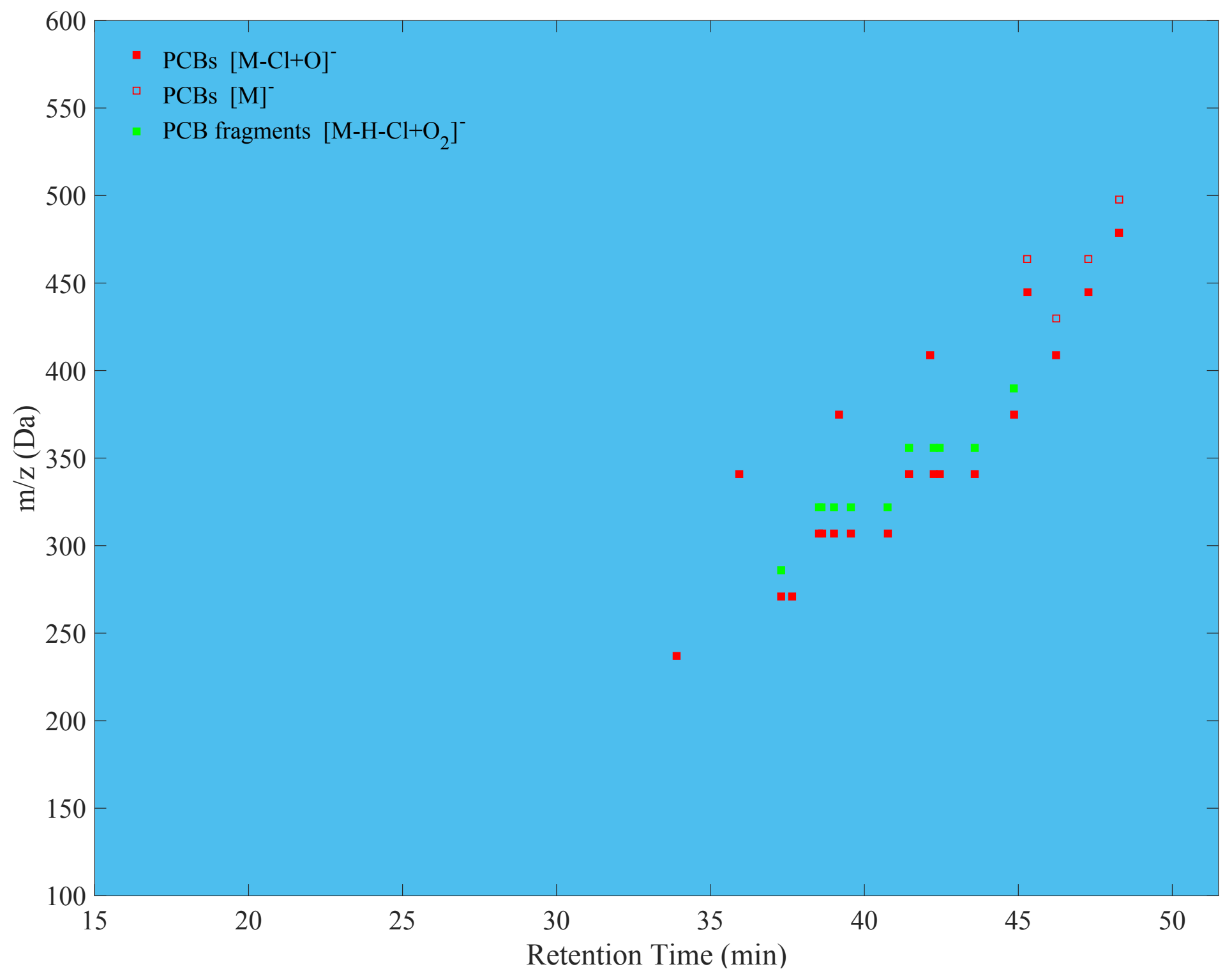

(a) 


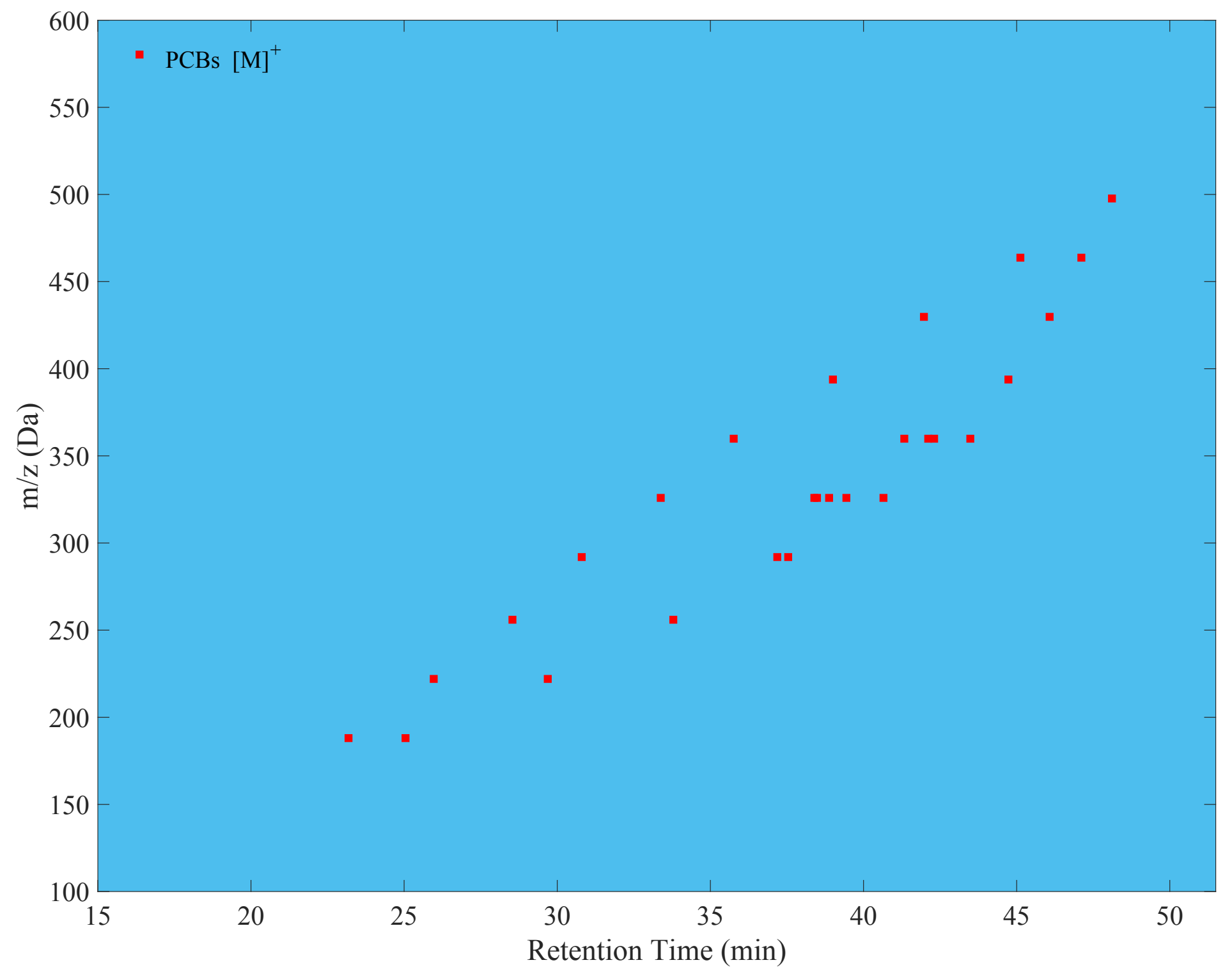

(b)

Figure S.12. Detected halogenated features by the IPDC algorithm in the 20pg of PCB standard mixture in (a) negative and (b) positive modes. 


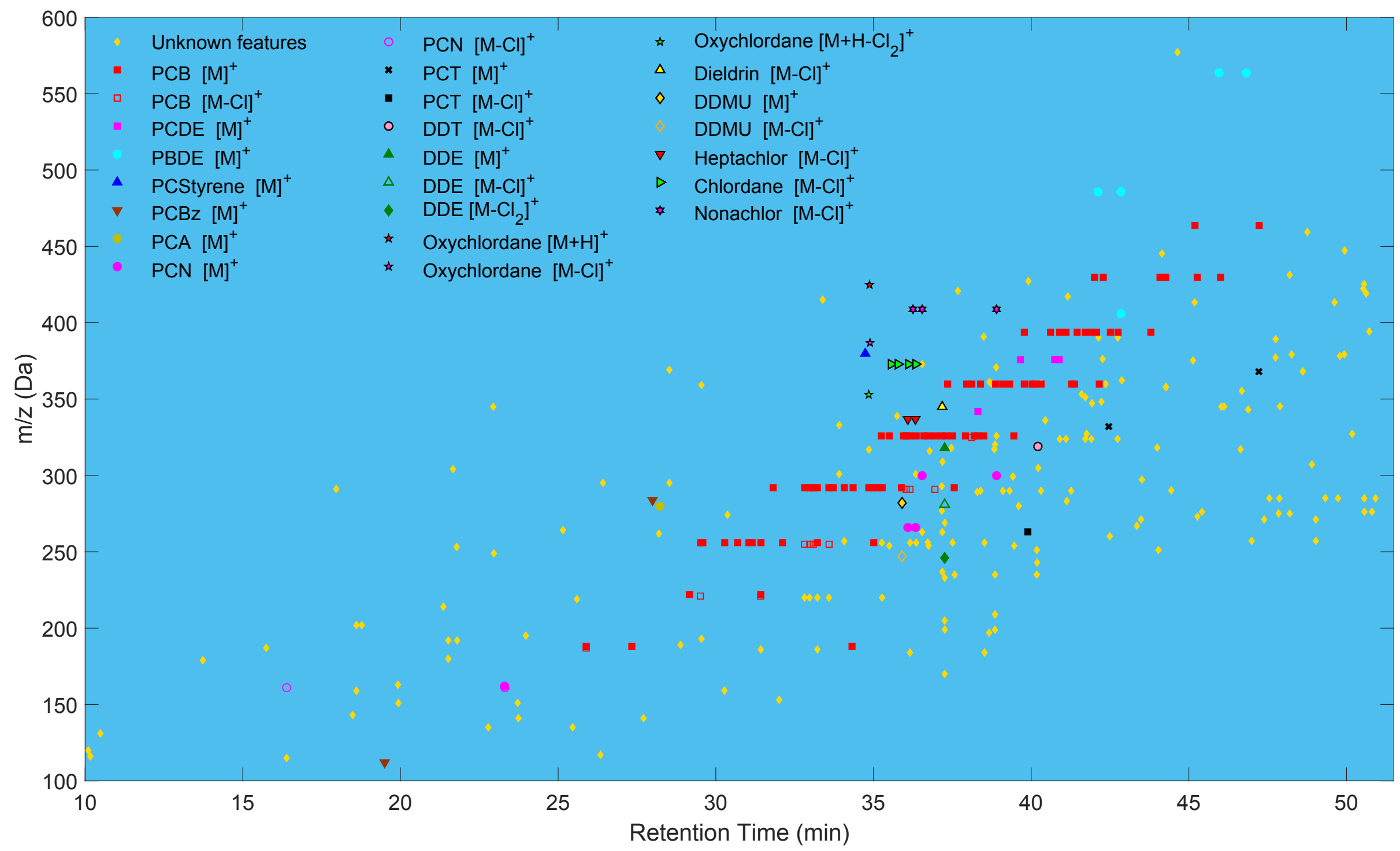

Figure S.13. Isotopic features in LMLT 2016 composite in positive mode. The identifications were based on isotopic model confirmation and previous knowledge of the presence. 
Table S.11. Summary of the IPDC algorithm results for the detected novel contaminants identified in LMLT 2014 (GC $\times$ GC-HRT) ${ }^{9}$ and in LMLT 2016 (Negative APGC-QToF). Additional molecular formula matches are provided for the negative APGC-QToF features. The negative APGC-

QToF candidate that matches the molecular formula identified by the $\mathrm{GC} \times \mathrm{GC}-\mathrm{ToF}$ is highlighted in green.

\begin{tabular}{|c|c|c|c|c|c|c|c|c|c|c|c|c|c|}
\hline \multicolumn{2}{|c|}{$\begin{array}{l}\text { Order of the candidate for } \\
\text { the isotopic feature }\end{array}$} & $\begin{array}{l}\text { Candidate } \\
\text { molecular ion } \\
\text { formula }\end{array}$ & $\begin{array}{c}\text { Detected } \\
\text { mass of } \\
\text { molecular } \\
\text { ion (Da) }\end{array}$ & $\begin{array}{c}\text { Exact } \\
\text { mass of } \\
\text { molecular } \\
\text { ion (Da) }\end{array}$ & Intensity & $\begin{array}{l}\text { NEME } \\
(\mathrm{mDa})\end{array}$ & $\begin{array}{l}\text { PCS } \\
(\%)\end{array}$ & $\begin{array}{l}\mathrm{RT} \\
(\min )\end{array}$ & $\begin{array}{l}\text { Peak } \\
\text { Area }\end{array}$ & $\begin{array}{l}\text { ND } \\
\text { CS }\end{array}$ & $\begin{array}{l}\mathrm{RCS} \\
(\%)\end{array}$ & RPW & $\begin{array}{c}\text { Peak } \\
\text { Identificat } \\
\text { ion score }\end{array}$ \\
\hline \multicolumn{14}{|c|}{$\mathrm{C}_{12} \mathrm{H}_{19} \mathrm{ClO}$} \\
\hline \multirow{2}{*}{ Isomer 1} & 1 & {$\left[\mathrm{C}_{10} \mathrm{H}_{17} \mathrm{ClN}_{3}\right]^{-}$} & 214.1138 & 214.1112 & 3700 & 6.1 & 977 & 23.45 & 429 & 20 & 42 & 0.38 & $6 \mathrm{E}+02$ \\
\hline & 2 & {$\left[\mathrm{C}_{12} \mathrm{H}_{19} \mathrm{ClO}\right]^{-}$} & 214.1138 & 214.1125 & 3700 & 3.9 & 971 & 23.45 & 371 & 18 & 53 & 0.52 & $5 \mathrm{E}+02$ \\
\hline & & & & & & & & & & & & & \\
\hline \multirow{5}{*}{ Isomer 2} & 1 & {$\left[\mathrm{C}_{12} \mathrm{H}_{18} \mathrm{ClO}\right]^{-}$} & 213.1018 & 213.1047 & 49519 & 1.2 & 966 & 23.81 & 4466 & 40 & 63 & 0.18 & $5.6 \mathrm{E}+03$ \\
\hline & 2 & {$\left[\mathrm{C}_{10} \mathrm{H}_{16} \mathrm{ClN}_{3}\right]^{-}$} & 213.1018 & 213.1033 & 49519 & 1.9 & 966 & 23.81 & 4511 & 38 & 58 & 0.17 & $5.2 \mathrm{E}+03$ \\
\hline & 3 & {$\left[\mathrm{C}_{11} \mathrm{H}_{17} \mathrm{ClNO}\right]^{-}$} & 214.1063 & 214.0999 & 8188 & 4.3 & 983 & 23.80 & 481 & 12 & 50 & 0.38 & $9.9 \mathrm{E}+02$ \\
\hline & 4 & {$\left[\mathrm{C}_{10} \mathrm{H}_{17} \mathrm{ClN}_{3}\right]^{-}$} & 214.1059 & 214.1112 & 9028 & 7.3 & 960 & 23.81 & 600 & 14 & 54 & 0.43 & $5.7 \mathrm{E}+02$ \\
\hline & 5 & {$\left[\mathrm{C}_{12} \mathrm{H}_{19} \mathrm{ClO}\right]$} & 214.1072 & 214.1125 & 7241 & 7.7 & 959 & 23.80 & 492 & 7 & 28 & 0.48 & $2.4 \mathrm{E}+02$ \\
\hline & & & & & & & & & & & & & \\
\hline \multirow{2}{*}{ Isomer 3} & 1 & {$\left[\mathrm{C}_{12} \mathrm{H}_{19} \mathrm{ClO}\right]^{-}$} & 214.1157 & 214.1125 & 13879 & 7.0 & 986 & 36.11 & 823 & 10 & 44 & 0.44 & $1.1 \mathrm{E}+03$ \\
\hline & 2 & [C10H17ClN3]- & 214.1157 & 214.1112 & 13879 & 6.7 & 981 & 36.11 & 823 & 10 & 44 & 0.44 & $9.7 \mathrm{E}+02$ \\
\hline \multicolumn{14}{|c|}{$\mathrm{C}_{9} \mathrm{H}_{11} \mathrm{ClO}$} \\
\hline \multirow{3}{*}{ Isomer 1} & 1 & {$\left[\mathrm{C}_{9} \mathrm{H}_{11} \mathrm{ClO}\right]^{-}$} & 170.0487 & 170.0499 & 40860 & 6.3 & 958 & 18.33 & 2782 & 15 & 54 & 0.45 & $1.1 \mathrm{E}+03$ \\
\hline & 2 & {$\left[\mathrm{C}_{7} \mathrm{H}_{10} \mathrm{ClN}_{3}\right]^{-}$} & 171.0544 & 171.0564 & 7208 & 1.2 & 965 & 18.31 & 906 & 24 & 58 & 0.63 & $8.4 \mathrm{E}+02$ \\
\hline & 3 & {$\left[\mathrm{C}_{7} \mathrm{H}_{9} \mathrm{ClN}_{3}\right]^{-}$} & 170.0487 & 170.0485 & 40860 & 6.5 & 951 & 18.33 & 2636 & 11 & 39 & 0.41 & $7.4 \mathrm{E}+02$ \\
\hline & & & & & & & & & & & & & \\
\hline \multirow{2}{*}{ Isomer 2} & 1 & {$\left[\mathrm{C}_{9} \mathrm{H}_{11} \mathrm{ClO}\right]^{-}$} & 170.0528 & 170.0499 & 30332 & 4.6 & 963 & 34.89 & 2779 & 22 & 55 & 0.39 & $1.7 \mathrm{E}+03$ \\
\hline & 2 & {$\left[\mathrm{C}_{7} \mathrm{H}_{9} \mathrm{ClN}_{3}\right]^{-}$} & 170.0520 & 170.0485 & 29047 & 5.2 & 962 & 34.90 & 2701 & 15 & 37 & 0.39 & $1.1 \mathrm{E}+03$ \\
\hline \multicolumn{14}{|c|}{$\mathrm{C}_{9} \mathrm{H}_{13} \mathrm{ClO}_{2}$} \\
\hline \multirow{3}{*}{ Isomer 1} & 1 & {$\left[\mathrm{C}_{9} \mathrm{H}_{13} \mathrm{ClO}_{2}\right]^{-}$} & 188.0617 & 188.0605 & 2595 & 3.2 & 978 & 18.70 & 513 & 40 & 52 & 0.40 & $9.3 \mathrm{E}+02$ \\
\hline & 2 & {$\left[\mathrm{C}_{7} \mathrm{H}_{11} \mathrm{ClN}_{3} \mathrm{O}\right]^{-}$} & 188.0616 & 188.0591 & 2783 & 4.1 & 984 & 18.65 & 318 & 25 & 65 & 0.52 & $7.3 \mathrm{E}+02$ \\
\hline & 3 & {$\left[\mathrm{C}_{9} \mathrm{H}_{14} \mathrm{ClP}\right]^{-}$} & 188.0616 & 188.0522 & 2783 & 5.0 & 983 & 18.65 & 560 & 23 & 34 & 0.60 & $5.6 \mathrm{E}+02$ \\
\hline
\end{tabular}




\begin{tabular}{|c|c|c|c|c|c|c|c|c|c|c|c|c|c|}
\hline & 4 & {$\left[\mathrm{C}_{7} \mathrm{H}_{11} \mathrm{ClN}_{3} \mathrm{O}\right]^{-}$} & 188.0617 & 188.0591 & 2595 & 3.2 & 975 & 18.70 & 408 & 26 & 51 & 0.59 & $5.1 \mathrm{E}+02$ \\
\hline \multicolumn{2}{|c|}{$\begin{array}{l}\text { Order of the candidate for } \\
\text { the isotopic feature }\end{array}$} & $\begin{array}{l}\text { Candidate } \\
\text { molecular ion } \\
\text { formula }\end{array}$ & $\begin{array}{c}\text { Detected } \\
\text { mass of } \\
\text { molecular } \\
\text { ion (Da) }\end{array}$ & $\begin{array}{c}\text { Exact } \\
\text { mass of } \\
\text { molecular } \\
\text { ion }(\mathrm{Da})\end{array}$ & Intensity & $\begin{array}{l}\text { NEME } \\
(\mathrm{mDa})\end{array}$ & $\begin{array}{l}\text { PCS } \\
(\%)\end{array}$ & $\begin{array}{c}\text { RT } \\
(\mathrm{min})\end{array}$ & $\begin{array}{l}\text { Peak } \\
\text { Area }\end{array}$ & $\begin{array}{l}\text { ND } \\
\text { CS }\end{array}$ & $\begin{array}{c}\mathrm{RCS} \\
(\%)\end{array}$ & RPW & $\begin{array}{c}\text { Peak } \\
\text { Identificat } \\
\text { ion score }\end{array}$ \\
\hline \multicolumn{14}{|c|}{$\mathrm{C}_{8} \mathrm{H}_{9} \mathrm{ClO}$} \\
\hline \multirow{3}{*}{ Isomer 1} & 1 & {$\left[\mathrm{C}_{7} \mathrm{H}_{8} \mathrm{ClNO}\right]^{-}$} & 157.0385 & 157.0295 & 2736 & 4.8 & 990 & 16.09 & 276 & 9 & 31 & 0.76 & $2.9 \mathrm{E}+02$ \\
\hline & 2 & {$\left[\mathrm{C}_{8} \mathrm{H}_{9} \mathrm{ClO}\right]^{-}$} & 156.0291 & 156.0342 & 8314 & 3.5 & 953 & 16.07 & 433 & 4 & 28 & 0.68 & $1.3 \mathrm{E}+02$ \\
\hline & 3 & {$\left[\mathrm{C}_{6} \mathrm{H}_{7} \mathrm{ClN}_{3}\right]^{-}$} & 156.0291 & 156.0329 & 8314 & 3.5 & 949 & 16.07 & 433 & 4 & 28 & 0.68 & $1.2 \mathrm{E}+02$ \\
\hline & & & & & & & & & & & & & \\
\hline \multirow{6}{*}{ Isomer 2} & 1 & {$\left[\mathrm{C}_{8} \mathrm{H}_{8} \mathrm{ClO}\right]^{-}$} & 155.0249 & 155.0264 & 75816 & 2.2 & 957 & 23.53 & 5895 & 20 & 33 & 0.21 & $2.3 \mathrm{E}+03$ \\
\hline & 2 & {$\left[\mathrm{C}_{6} \mathrm{H}_{6} \mathrm{ClN}_{3}\right]^{-}$} & 155.0249 & 155.0250 & 75816 & 2.0 & 955 & 23.53 & 5895 & 20 & 33 & 0.21 & $2.1 \mathrm{E}+03$ \\
\hline & 3 & {$\left[\mathrm{C}_{6} \mathrm{H}_{7} \mathrm{ClN}_{3}\right]^{-}$} & 156.0283 & 156.0329 & 7944 & 2.8 & 959 & 23.54 & 802 & 13 & 37 & 0.67 & $3.9 \mathrm{E}+02$ \\
\hline & 4 & {$\left[\mathrm{C}_{7} \mathrm{H}_{7} \mathrm{ClNO}\right]^{-}$} & 156.0283 & 156.0216 & 7944 & 5.7 & 965 & 23.54 & 791 & 10 & 29 & 0.60 & $3.6 \mathrm{E}+02$ \\
\hline & 5 & {$\left[\mathrm{C}_{8} \mathrm{H}_{9} \mathrm{ClO}\right]^{-}$} & 156.0283 & 156.0342 & 7944 & 3.7 & 962 & 23.54 & 612 & 9 & 38 & 0.76 & $3.0 \mathrm{E}+02$ \\
\hline & 6 & {$\left[\mathrm{C}_{4} \mathrm{H}_{9} \mathrm{ClO}_{4}\right]^{-}$} & 156.0273 & 156.0190 & 5887 & 5.3 & 959 & 23.55 & 684 & 9 & 26 & 0.74 & $2.1 \mathrm{E}+02$ \\
\hline \multicolumn{14}{|c|}{$\mathrm{C}_{9} \mathrm{H}_{11} \mathrm{ClO}$} \\
\hline \multirow{3}{*}{ Isomer 1} & 1 & {$\left[\mathrm{C}_{9} \mathrm{H}_{11} \mathrm{ClO}\right]^{-}$} & 170.0487 & 170.0499 & 40860 & 6.3 & 958 & 18.33 & 2782 & 15 & 54 & 0.45 & $1.1 \mathrm{E}+03$ \\
\hline & 2 & {$\left[\mathrm{C}_{7} \mathrm{H}_{10} \mathrm{ClN}_{3}\right]^{-}$} & 171.0544 & 171.0564 & 7208 & 1.2 & 965 & 18.31 & 906 & 24 & 58 & 0.63 & $8.4 \mathrm{E}+02$ \\
\hline & 3 & {$\left[\mathrm{C}_{7} \mathrm{H}_{9} \mathrm{ClN}_{3}\right]^{-}$} & 170.0487 & 170.0485 & 40860 & 6.5 & 951 & 18.33 & 2636 & 11 & 39 & 0.41 & $7.4 \mathrm{E}+02$ \\
\hline \multirow{3}{*}{ Isomer 2} & & & & & & & & & & & & & \\
\hline & 1 & {$\left[\mathrm{C}_{9} \mathrm{H}_{11} \mathrm{ClO}\right]^{-}$} & 170.0528 & 170.0499 & 30332 & 4.6 & 963 & 34.89 & 2779 & 22 & 55 & 0.39 & $1.7 \mathrm{E}+03$ \\
\hline & 2 & {$\left[\mathrm{C}_{7} \mathrm{H}_{9} \mathrm{ClN}_{3}\right]^{-}$} & 170.0520 & 170.0485 & 29047 & 5.2 & 962 & 34.90 & 2701 & 15 & 37 & 0.39 & $1.1 \mathrm{E}+03$ \\
\hline \multicolumn{14}{|c|}{$\mathrm{C}_{8} \mathrm{H}_{9} \mathrm{ClO}_{2}$} \\
\hline \multirow{5}{*}{ Isomer 1} & 1 & {$\left[\mathrm{C}_{7} \mathrm{H}_{8} \mathrm{ClN}_{2} \mathrm{O}\right]^{-}$} & 171.0359 & 171.0325 & 66481 & 5.7 & 972 & 16.48 & 4198 & 17 & 67 & 0.46 & $2.4 \mathrm{E}+03$ \\
\hline & 2 & {$\left[\mathrm{C}_{7} \mathrm{H}_{8} \mathrm{ClN}_{2} \mathrm{O}\right]^{-}$} & 171.0358 & 171.0325 & 68707 & 5.4 & 970 & 16.53 & 3526 & 15 & 74 & 0.46 & $2.2 \mathrm{E}+03$ \\
\hline & 3 & {$\left[\mathrm{C}_{7} \mathrm{H}_{9} \mathrm{ClN}_{2} \mathrm{O}\right]^{-}$} & 172.0387 & 172.0404 & 12213 & 2.8 & 983 & 16.50 & 1210 & 26 & 78 & 0.54 & $2.1 \mathrm{E}+03$ \\
\hline & 4 & {$\left[\mathrm{C}_{8} \mathrm{H}_{10} \mathrm{ClNO}\right]^{-}$} & 171.0358 & 171.0451 & 51786 & 7.8 & 962 & 16.45 & 1920 & 8 & 36 & 0.27 & $1.0 \mathrm{E}+03$ \\
\hline & 5 & {$\left[\mathrm{C}_{8} \mathrm{H}_{9} \mathrm{ClO}_{2}\right]^{-}$} & 172.0387 & 172.0291 & 12213 & 7.6 & 965 & 16.50 & 945 & 9 & 30 & 0.48 & $4.7 \mathrm{E}+02$ \\
\hline
\end{tabular}




\begin{tabular}{|c|c|c|c|c|c|c|c|c|c|c|c|c|c|}
\hline \multicolumn{2}{|c|}{$\begin{array}{l}\text { Order of the candidate for } \\
\text { the isotopic feature }\end{array}$} & \multirow{2}{*}{ 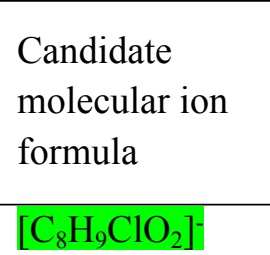 } & \multirow{2}{*}{$\begin{array}{c}\text { Detected } \\
\text { mass of } \\
\text { molecular } \\
\text { ion }(\mathrm{Da}) \\
172.0322\end{array}$} & \multirow{2}{*}{$\begin{array}{c}\text { Exact } \\
\text { mass of } \\
\text { molecular } \\
\text { ion }(\mathrm{Da}) \\
172.0291 \\
\end{array}$} & \multirow{2}{*}{$\begin{array}{c}\text { Intensity } \\
6594\end{array}$} & \multirow{2}{*}{$\begin{array}{c}\text { NEME } \\
(\mathrm{mDa})\end{array}$} & \multirow{2}{*}{$\begin{array}{l}\text { PCS } \\
(\%)\end{array}$} & \multirow{2}{*}{$\begin{array}{c}\mathrm{RT} \\
(\mathrm{min}) \\
33.98\end{array}$} & \multirow{2}{*}{$\begin{array}{r}\text { Peak } \\
\text { Area } \\
623\end{array}$} & \multirow{2}{*}{$\begin{array}{l}\text { ND } \\
\text { CS } \\
13\end{array}$} & \multirow{2}{*}{$\begin{array}{r}\mathrm{RCS} \\
(\%) \\
43\end{array}$} & \multirow{2}{*}{$\begin{array}{l}\text { RPW } \\
0.55\end{array}$} & \multirow{2}{*}{$\begin{array}{c}\begin{array}{c}\text { Peak } \\
\text { Identificat } \\
\text { ion score }\end{array} \\
3.4 \mathrm{E}+02\end{array}$} \\
\hline \multirow{3}{*}{ Isomer 2} & 1 & & & & & & & & & & & & \\
\hline & 2 & {$\left[\mathrm{C}_{6} \mathrm{H}_{7} \mathrm{ClN}_{3} \mathrm{O}\right]^{-}$} & 172.0322 & 172.0278 & 6594 & 4.2 & 948 & 33.98 & 600 & 11 & 36 & 0.55 & $2.4 \mathrm{E}+02$ \\
\hline & 3 & {$\left[\mathrm{C}_{10} \mathrm{H}_{7} \mathrm{ClN}\right]^{-}$} & 176.0291 & 176.0267 & 5525 & 6.0 & 893 & 34.00 & 509 & 11 & 36 & 0.61 & $4.0 \mathrm{E}+01$ \\
\hline \multirow{3}{*}{ Isomer 3} & & & & & & & & & & & & & \\
\hline & 1 & {$\left[\mathrm{C}_{8} \mathrm{H}_{9} \mathrm{ClO}_{2}\right]^{-}$} & 172.0313 & 172.0291 & 6812 & 5.0 & 947 & 37.33 & 656 & 9 & 32 & 0.76 & $1.7 \mathrm{E}+02$ \\
\hline & 2 & {$\left[\mathrm{C}_{6} \mathrm{H}_{7} \mathrm{ClN}_{3} \mathrm{O}\right]^{-}$} & 172.0313 & 172.0278 & 6812 & 5.3 & 942 & 37.33 & 656 & 9 & 32 & 0.76 & $1.5 \mathrm{E}+02$ \\
\hline \multicolumn{14}{|c|}{$\mathrm{C}_{11} \mathrm{H}_{17} \mathrm{ClO}_{2}$} \\
\hline \multirow{3}{*}{ Isomer 1} & 1 & {$\left[\mathrm{C}_{11} \mathrm{H}_{17} \mathrm{ClO}_{2}\right]^{-}$} & 216.0940 & 216.0918 & 3649 & 2.8 & 975 & 18.75 & 355 & 26 & 72 & 0.42 & $8.6 \mathrm{E}+02$ \\
\hline & 2 & {$\left[\mathrm{C}_{9} \mathrm{H}_{15} \mathrm{ClN}_{3} \mathrm{O}\right]^{-}$} & 216.0940 & 216.0904 & 3649 & 3.1 & 974 & 18.75 & 352 & 24 & 67 & 0.41 & $7.9 \mathrm{E}+02$ \\
\hline & 3 & {$\left[\mathrm{C}_{10} \mathrm{H}_{17} \mathrm{ClN}_{2} \mathrm{O}\right]^{-}$} & 216.0940 & 216.1030 & 3649 & 7.0 & 966 & 18.75 & 342 & 8 & 27 & 0.57 & $1.8 \mathrm{E}+02$ \\
\hline \multirow{3}{*}{ Isomer 2} & & & & & & & & & & & & & \\
\hline & 1 & {$\left[\mathrm{C}_{11} \mathrm{H}_{17} \mathrm{ClO}_{2}\right]^{-}$} & 216.0929 & 216.0918 & 2532 & 1.9 & 976 & 19.00 & 327 & 39 & 76 & 0.52 & $8.2 \mathrm{E}+02$ \\
\hline & 2 & {$\left[\mathrm{C}_{9} \mathrm{H}_{15} \mathrm{ClN}_{3} \mathrm{O}\right]^{-}$} & 216.0929 & 216.0904 & 2532 & 2.5 & 975 & 19.00 & 324 & 37 & 72 & 0.46 & $7.8 \mathrm{E}+02$ \\
\hline \multirow{5}{*}{ Isomer 3} & 1 & {$\left[\mathrm{C}_{11} \mathrm{H}_{17} \mathrm{ClO}_{2}\right]^{-}$} & 216.0964 & 216.0918 & 16973 & 3.6 & 945 & 39.62 & 1211 & 14 & 59 & 0.58 & $5.0 \mathrm{E}+02$ \\
\hline & 2 & {$\left[\mathrm{C}_{9} \mathrm{H}_{15} \mathrm{ClN}_{3} \mathrm{O}\right]^{-}$} & 216.0964 & 216.0904 & 16973 & 4.0 & 941 & 39.62 & 1208 & 13 & 55 & 0.58 & $4.2 \mathrm{E}+02$ \\
\hline & 3 & {$\left[\mathrm{C}_{14} \mathrm{H}_{15} \mathrm{Cl}\right]^{-}$} & 218.0841 & 218.0863 & 8606 & 6.9 & 966 & 39.60 & 493 & 6 & 36 & 0.67 & $2.7 \mathrm{E}+02$ \\
\hline & 4 & {$\left[\mathrm{C}_{11} \mathrm{H}_{19} \mathrm{ClS}\right]^{-}$} & 218.0841 & 218.0897 & 8606 & 6.4 & 962 & 39.60 & 322 & 4 & 34 & 0.60 & $1.8 \mathrm{E}+02$ \\
\hline & 5 & {$\left[\mathrm{C}_{10} \mathrm{H}_{18} \mathrm{ClNP}\right]^{-}$} & 218.0841 & 218.0866 & 8606 & 6.7 & 958 & 39.60 & 429 & 4 & 28 & 0.68 & $1.5 \mathrm{E}+02$ \\
\hline \multicolumn{14}{|c|}{ Chlorophenyl-pyrimidine $\left(\mathrm{C}_{10} \mathrm{H}_{7} \mathrm{ClN}_{2}\right)$} \\
\hline \multirow{6}{*}{ Isomer 1} & 1 & {$\left[\mathrm{C}_{11} \mathrm{H}_{7} \mathrm{ClO}\right]^{-}$} & 190.0199 & 190.0186 & 19379 & 5.6 & 976 & 31.02 & 1219 & 15 & 60 & 0.45 & $1.4 \mathrm{E}+03$ \\
\hline & 2 & {$\left[\mathrm{C}_{9} \mathrm{H}_{5} \mathrm{ClN}_{3}\right]^{-}$} & 190.0199 & 190.0172 & 19379 & 6.6 & 972 & 31.02 & 1219 & 15 & 61 & 0.45 & $1.2 \mathrm{E}+03$ \\
\hline & 3 & {$\left[\mathrm{C}_{8} \mathrm{H}_{11} \mathrm{ClOS}\right]^{-}$} & 190.0199 & 190.0220 & 19379 & 3.6 & 966 & 31.02 & 1248 & 14 & 53 & 0.41 & $1.1 \mathrm{E}+03$ \\
\hline & 4 & {$\left[\mathrm{C}_{7} \mathrm{H}_{9} \mathrm{ClNO}_{3}\right]^{-}$} & 190.0199 & 190.0271 & 19379 & 5.5 & 967 & 31.02 & 1216 & 12 & 49 & 0.44 & $9.3 \mathrm{E}+02$ \\
\hline & 5 & {$\left[\mathrm{C}_{7} \mathrm{H}_{10} \mathrm{ClNOP}\right]^{-}$} & 190.0199 & 190.0189 & 19379 & 5.3 & 966 & 31.02 & 1216 & 12 & 49 & 0.44 & $9.1 \mathrm{E}+02$ \\
\hline & 6 & {$\left[\mathrm{C}_{10} \mathrm{H}_{7} \mathrm{ClN}_{2}\right]^{-}$} & 190.0199 & 190.0298 & 19379 & 7.7 & 976 & 31.02 & 1022 & 8 & 41 & 0.49 & $8.1 \mathrm{E}+02$ \\
\hline
\end{tabular}




\begin{tabular}{|c|c|c|c|c|c|c|c|c|c|c|c|c|c|}
\hline & 7 & {$\left[\mathrm{C}_{6} \mathrm{H}_{7} \mathrm{ClN}_{2} \mathrm{O}_{3}\right]^{-}$} & 190.0199 & 190.0145 & 19379 & 8.7 & 962 & 31.02 & 1204 & 11 & 45 & 0.44 & $7.1 \mathrm{E}+02$ \\
\hline & 8 & {$\left[\mathrm{C}_{6} \mathrm{H}_{9} \mathrm{ClN}_{3} \mathrm{~S}\right]^{-}$} & 190.0199 & 190.0206 & 19379 & 4.2 & 961 & 31.02 & 1215 & 10 & 41 & 0.44 & $6.8 \mathrm{E}+02$ \\
\hline & 9 & {$\left[\mathrm{C}_{10} \mathrm{H}_{7} \mathrm{ClNO}\right]^{-}$} & 192.0253 & 192.0216 & 2472 & 3.2 & 970 & 31.09 & 124 & 4 & 27 & 0.50 & $9.6 \mathrm{E}+01$ \\
\hline & 10 & {$\left[\mathrm{C}_{8} \mathrm{H}_{10} \mathrm{Cl}_{2} \mathrm{~N}\right]^{-}$} & 190.0197 & 190.0191 & 4568 & 4.4 & 922 & 31.08 & 180 & 4 & 30 & 0.48 & $9.3 \mathrm{E}+01$ \\
\hline & & & & & & & & & & & & & \\
\hline \multicolumn{2}{|c|}{$\begin{array}{l}\text { Order of the candidate for } \\
\text { the isotopic feature }\end{array}$} & $\begin{array}{l}\text { Candidate } \\
\text { molecular ion } \\
\text { formula }\end{array}$ & $\begin{array}{c}\text { Detected } \\
\text { mass of } \\
\text { molecular } \\
\text { ion (Da) }\end{array}$ & $\begin{array}{l}\text { Exact mass } \\
\text { of } \\
\text { molecular } \\
\text { ion (Da) }\end{array}$ & Intensity & $\begin{array}{l}\text { NEME } \\
(\mathrm{mDa})\end{array}$ & $\begin{array}{l}\text { PCS } \\
(\%)\end{array}$ & $\begin{array}{l}\mathrm{RT} \\
(\mathrm{min})\end{array}$ & $\begin{array}{l}\text { Peak } \\
\text { Area }\end{array}$ & $\begin{array}{l}\text { ND } \\
\text { CS }\end{array}$ & $\begin{array}{l}\mathrm{RCS} \\
(\%)\end{array}$ & RPW & $\begin{array}{c}\text { Peak } \\
\text { Identificat } \\
\text { ion score }\end{array}$ \\
\hline \multicolumn{14}{|c|}{ 1,2,4,5-tetrachloro-3,6-dimethoxybenzene $\left(\mathrm{C}_{8} \mathrm{H}_{6} \mathrm{Cl}_{4} \mathrm{O}_{2}\right)$} \\
\hline \multirow{10}{*}[\mathrm{M}-\mathrm{Cl}+\mathrm{O}]{$^{-}$} & 1 & {$\left[\mathrm{C}_{4} \mathrm{H}_{8} \mathrm{Cl}_{3} \mathrm{NO}_{3} \mathrm{~S}\right]^{-}$} & 256.9282 & 256.9261 & 1099 & 4.6 & 999 & 28.36 & 36 & 5 & 71 & 0.41 & $6.1 \mathrm{E}+02$ \\
\hline & 2 & {$\left[\mathrm{C}_{5} \mathrm{H}_{11} \mathrm{Cl}_{3} \mathrm{OPS}\right]^{-}$} & 256.9282 & 256.9305 & 1099 & 0.7 & 999 & 28.36 & 38 & 7 & 94 & 0.41 & $6.0 \mathrm{E}+02$ \\
\hline & 3 & {$\left[\mathrm{C}_{7} \mathrm{H}_{6} \mathrm{Cl}_{3} \mathrm{~N}_{2} \mathrm{~S}\right]^{-}$} & 256.9282 & 256.9288 & 1099 & 2.0 & 999 & 28.36 & 38 & 7 & 94 & 0.41 & $5.5 \mathrm{E}+02$ \\
\hline & 4 & {$\left[\mathrm{C}_{8} \mathrm{H}_{7} \mathrm{Cl}_{3} \mathrm{OP}\right]^{-}$} & 254.9348 & 254.9300 & 1001 & 3.0 & 999 & 28.36 & 44 & 8 & 89 & 0.45 & $5.4 \mathrm{E}+02$ \\
\hline & 5 & {$\left[\mathrm{C}_{6} \mathrm{H}_{4} \mathrm{Cl}_{3} \mathrm{~N}_{3} \mathrm{O}_{2}\right]^{-}$} & 254.9348 & 254.9369 & 1001 & 2.2 & 998 & 28.36 & 44 & 8 & 89 & 0.45 & $5.3 \mathrm{E}+02$ \\
\hline & 6 & {$\left[\mathrm{C}_{8} \mathrm{H}_{6} \mathrm{Cl}_{3} \mathrm{O}_{3}\right]^{-}$} & 254.9348 & 254.9383 & 1001 & 3.2 & 999 & 28.36 & 44 & 8 & 89 & 0.45 & $5.2 \mathrm{E}+02$ \\
\hline & 7 & {$\left[\mathrm{C}_{4} \mathrm{H}_{9} \mathrm{Cl}_{3} \mathrm{NO}_{3} \mathrm{P}\right]^{-}$} & 254.9348 & 254.9386 & 1001 & 3.5 & 998 & 28.36 & 44 & 8 & 89 & 0.45 & $5.1 \mathrm{E}+02$ \\
\hline & 8 & {$\left[\mathrm{C}_{6} \mathrm{H}_{5} \mathrm{Cl}_{3} \mathrm{~N}_{3} \mathrm{P}\right]^{-}$} & 254.9348 & 254.9287 & 1001 & 4.0 & 1000 & 28.36 & 44 & 7 & 77 & 0.45 & $4.6 \mathrm{E}+02$ \\
\hline & 9 & {$\left[\mathrm{C}_{10} \mathrm{H}_{2} \mathrm{Cl}_{3} \mathrm{~N}_{2}\right]^{-}$} & 254.9348 & 254.9284 & 1001 & 4.3 & 999 & 28.36 & 44 & 7 & 77 & 0.45 & $4.6 \mathrm{E}+02$ \\
\hline & 10 & {$\left[\mathrm{C}_{5} \mathrm{H}_{9} \mathrm{Cl}_{4} \mathrm{NO}_{2}\right]^{-}$} & 256.9282 & 256.9358 & 1099 & 3.4 & 988 & 28.36 & 41 & 8 & 99 & 0.42 & $4.5 \mathrm{E}+02$ \\
\hline \multicolumn{14}{|c|}{ 2,4'-dichlorobenzophenone $\left(\mathrm{C}_{13} \mathrm{H}_{8} \mathrm{Cl}_{2} \mathrm{O}\right)$} \\
\hline \multirow{9}{*}{$\mathrm{M}^{-} \mathrm{Cl}+\mathrm{O}^{-}$} & 1 & {$\left[\mathrm{C}_{13} \mathrm{H}_{9} \mathrm{ClP}\right]^{-}$} & 231.0162 & 231.0131 & 128859 & 2.6 & 994 & 37.43 & 12336 & 181 & 78 & 0.06 & $6.8 \mathrm{E}+04$ \\
\hline & 2 & {$\left[\mathrm{C}_{10} \mathrm{H}_{13} \mathrm{ClPS}\right]^{-}$} & 231.0162 & 231.0165 & 128859 & 1.4 & 984 & 37.43 & 12437 & 185 & 79 & 0.06 & $5.5 \mathrm{E}+04$ \\
\hline & 3 & {$\left[\mathrm{C}_{11} \mathrm{H}_{6} \mathrm{ClN}_{3} \mathrm{O}\right]^{-}$} & 231.0162 & 231.0200 & 128859 & 3.5 & 989 & 37.43 & 12171 & 163 & 76 & 0.06 & $5.5 \mathrm{E}+04$ \\
\hline & 4 & {$\left[\mathrm{C}_{13} \mathrm{H}_{8} \mathrm{ClO}_{2}\right]^{-}$} & 231.0162 & 231.0213 & 128859 & 4.8 & 992 & 37.43 & 12123 & 155 & 76 & 0.07 & $5.1 \mathrm{E}+04$ \\
\hline & 5 & {$\left[\mathrm{C}_{8} \mathrm{H}_{8} \mathrm{ClN}_{2} \mathrm{O}_{4}\right]^{-}$} & 231.0162 & 231.0173 & 128859 & 1.5 & 985 & 37.43 & 12134 & 154 & 75 & 0.07 & $4.7 \mathrm{E}+04$ \\
\hline & 6 & {$\left[\mathrm{C}_{6} \mathrm{H}_{13} \mathrm{ClO}_{5} \mathrm{P}\right]^{-}$} & 231.0162 & 231.0190 & 128859 & 2.7 & 982 & 37.43 & 12177 & 154 & 72 & 0.06 & $4.4 \mathrm{E}+04$ \\
\hline & 7 & {$\left[\mathrm{C}_{9} \mathrm{H}_{11} \mathrm{ClNO}_{2} \mathrm{P}\right]^{-}$} & 231.0162 & 231.0216 & 128859 & 5.1 & 987 & 37.43 & 12141 & 148 & 73 & 0.07 & $4.3 \mathrm{E}+04$ \\
\hline & 8 & {$\left[\mathrm{C}_{12} \mathrm{H}_{6} \mathrm{ClNO}_{2}\right]^{-}$} & 231.0162 & 231.0087 & 128859 & 5.7 & 995 & 37.43 & 11627 & 103 & 63 & 0.08 & $3.9 \mathrm{E}+04$ \\
\hline & 9 & {$\left[\mathrm{C}_{8} \mathrm{H}_{9} \mathrm{ClN}_{2} \mathrm{O}_{2} \mathrm{P}\right]^{-}$} & 231.0162 & 231.0091 & 128859 & 5.4 & 991 & 37.43 & 11620 & 106 & 65 & 0.08 & $3.5 \mathrm{E}+04$ \\
\hline
\end{tabular}




\begin{tabular}{|c|c|c|c|c|c|c|c|c|c|c|c|c|c|}
\hline & 10 & {$\left[\mathrm{C}_{8} \mathrm{H}_{10} \mathrm{ClN}_{3} \mathrm{OS}\right]^{-}$} & 231.0162 & 231.0233 & 128859 & 6.7 & 981 & 37.43 & 12145 & 138 & 68 & 0.07 & $3.3 \mathrm{E}+04$ \\
\hline \multicolumn{2}{|c|}{$\begin{array}{l}\text { Order of the candidate for } \\
\text { the isotopic feature }\end{array}$} & $\begin{array}{l}\text { Candidate } \\
\text { molecular ion } \\
\text { formula }\end{array}$ & $\begin{array}{c}\text { Detected } \\
\text { mass of } \\
\text { molecular } \\
\text { ion }(\mathrm{Da})\end{array}$ & $\begin{array}{l}\text { Exact mass } \\
\text { of } \\
\text { molecular } \\
\text { ion (Da) }\end{array}$ & Intensity & $\begin{array}{c}\text { NEM } \\
\text { E } \\
(\mathrm{mDa} \\
)\end{array}$ & $\begin{array}{l}\text { PCS } \\
(\% \%)\end{array}$ & $\begin{array}{l}\mathrm{RT} \\
(\mathrm{min})\end{array}$ & $\begin{array}{l}\text { Peak } \\
\text { Area }\end{array}$ & $\begin{array}{l}\text { ND } \\
\text { CS }\end{array}$ & $\begin{array}{l}\mathrm{RCS} \\
(\%)\end{array}$ & RPW & $\begin{array}{c}\text { Peak } \\
\text { Identificat } \\
\text { ion score }\end{array}$ \\
\hline \multirow{10}{*}[\mathrm{M}]{$^{-}$} & 1 & {$\left[\mathrm{C}_{9} \mathrm{H}_{12} \mathrm{Cl}_{2} \mathrm{NOP}\right]^{-}$} & 250.9976 & 251.0034 & 3767 & 7.2 & 993 & 37.51 & 235 & 7 & 28 & 0.33 & $1.0 \mathrm{E}+03$ \\
\hline & 2 & {$\left[\mathrm{C}_{14} \mathrm{H}_{5} \mathrm{ClNP}\right]^{-}$} & 252.9920 & 252.9848 & 2892 & 4.9 & 985 & 37.51 & 160 & 5 & 27 & 0.46 & $1.9 \mathrm{E}+02$ \\
\hline & 3 & {$\left[\mathrm{C}_{8} \mathrm{H}_{13} \mathrm{BrClN}_{2}\right]^{-}$} & 252.9932 & 252.9929 & 2760 & 3.3 & 940 & 37.50 & 154 & 6 & 32 & 0.42 & $1.5 \mathrm{E}+02$ \\
\hline & 4 & {$\left[\mathrm{C}_{8} \mathrm{H}_{14} \mathrm{Cl}_{4}\right]^{-}$} & 251.9847 & 251.9821 & 1298 & 5.0 & 950 & 37.58 & 49 & 3 & 31 & 0.46 & $7.5 \mathrm{E}+01$ \\
\hline & 5 & {$\left[\mathrm{C}_{13} \mathrm{H}_{8} \mathrm{Cl}_{2} \mathrm{O}\right]^{-}$} & 249.9913 & 249.9953 & 1524 & 4.4 & 944 & 37.60 & 69 & 3 & 26 & 0.53 & $4.3 \mathrm{E}+01$ \\
\hline & 6 & {$\left[\mathrm{C}_{8} \mathrm{H}_{8} \mathrm{Cl}_{2} \mathrm{NO}_{4}\right]^{-}$} & 251.9847 & 251.9831 & 1298 & 5.3 & 931 & 37.58 & 50 & 3 & 30 & 0.48 & $2.3 \mathrm{E}+01$ \\
\hline & 7 & {$\left[\mathrm{C}_{14} \mathrm{H}_{3} \mathrm{ClN}_{2} \mathrm{O}\right]^{-}$} & 249.9927 & 249.9934 & 1604 & 2.6 & 916 & 37.59 & 129 & 8 & 33 & 0.60 & $2.1 \mathrm{E}+01$ \\
\hline & 8 & {$\left[\mathrm{C}_{11} \mathrm{H}_{6} \mathrm{Cl}_{2} \mathrm{~N}_{2} \mathrm{O}\right]^{-}$} & 251.9847 & 251.9857 & 1298 & 6.0 & 913 & 37.58 & 50 & 4 & 41 & 0.48 & $1.7 \mathrm{E}+01$ \\
\hline & 9 & {$\left[\mathrm{C}_{11} \mathrm{H}_{9} \mathrm{BrNO}\right]^{-}$} & 249.9927 & 249.9868 & 1604 & 3.4 & 907 & 37.59 & 56 & 3 & 32 & 0.42 & $1.7 \mathrm{E}+01$ \\
\hline & 10 & {$\left[\mathrm{C}_{12} \mathrm{H}_{7} \mathrm{ClO}_{2} \mathrm{~S}\right]^{-}$} & 249.9927 & 249.9856 & 1604 & 4.4 & 899 & 37.59 & 70 & 3 & 28 & 0.69 & $5.0 \mathrm{E}+00$ \\
\hline \multicolumn{14}{|c|}{ Monochloromethoxyphenol $\left(\mathrm{C}_{7} \mathrm{H}_{7} \mathrm{ClO}_{2}\right)$} \\
\hline \multirow{3}{*}{ Isomer 1} & 1 & {$\left[\mathrm{C}_{7} \mathrm{H}_{7} \mathrm{ClO}_{2}\right]^{-}$} & 158.0110 & 158.0135 & 2095 & 4.8 & 959 & 14.53 & 112 & 7 & 47 & 0.46 & $1.0 \mathrm{E}+02$ \\
\hline & 2 & {$\left[\mathrm{C}_{5} \mathrm{H}_{5} \mathrm{ClN}_{3} \mathrm{O}\right]^{-}$} & 158.0110 & 158.0121 & 2095 & 3.6 & 953 & 14.53 & 112 & 7 & 47 & 0.46 & $8.9 \mathrm{E}+01$ \\
\hline & 3 & {$\left[\mathrm{C}_{7} \mathrm{H}_{8} \mathrm{ClP}\right]^{-}$} & 158.0110 & 158.0053 & 2095 & 4.4 & 954 & 14.53 & 67 & 3 & 33 & 0.40 & $4.1 \mathrm{E}+01$ \\
\hline & & & & & & & & & & & & & \\
\hline \multirow{3}{*}{ Isomer 2} & 1 & {$\left[\mathrm{C}_{7} \mathrm{H}_{7} \mathrm{ClO}_{2}\right]^{-}$} & 158.0122 & 158.0135 & 3576 & 4.5 & 950 & 15.26 & 131 & 5 & 41 & 0.41 & $1.0 \mathrm{E}+02$ \\
\hline & 2 & {$\left[\mathrm{C}_{5} \mathrm{H}_{5} \mathrm{ClN}_{3} \mathrm{O}\right]^{-}$} & 158.0122 & 158.0121 & 3576 & 3.7 & 948 & 15.26 & 131 & 5 & 41 & 0.41 & $9.5 \mathrm{E}+01$ \\
\hline & 3 & {$\left[\mathrm{C}_{7} \mathrm{H}_{8} \mathrm{ClP}\right]^{-}$} & 158.0121 & 158.0053 & 2562 & 5.6 & 950 & 15.25 & 85 & 4 & 40 & 0.42 & $5.8 \mathrm{E}+01$ \\
\hline & & & & & & & & & & & & & \\
\hline \multirow{2}{*}{ Isomer 3} & 1 & {$\left[\mathrm{C}_{7} \mathrm{H}_{7} \mathrm{ClO}_{2}\right]^{-}$} & 158.0164 & 158.0135 & 4172 & 3.7 & 943 & 30.40 & 311 & 9 & 39 & 0.56 & $1.3 \mathrm{E}+02$ \\
\hline & 2 & {$\left[\mathrm{C}_{5} \mathrm{H}_{5} \mathrm{ClN}_{3} \mathrm{O}\right]^{-}$} & 158.0164 & 158.0121 & 4172 & 3.8 & 942 & 30.40 & 298 & 7 & 31 & 0.54 & $1.0 \mathrm{E}+02$ \\
\hline \multicolumn{14}{|c|}{ Dichloromethoxyphenol $\left(\mathrm{C}_{7} \mathrm{H}_{6} \mathrm{Cl}_{2} \mathrm{O}_{2}\right)$} \\
\hline \multirow{3}{*}[\mathrm{M}-\mathrm{Cl}+\mathrm{O}]{$^{-}$} & 1 & {$\left[\mathrm{C}_{7} \mathrm{H}_{7} \mathrm{ClOP}\right]^{-}$} & 172.9935 & 172.9923 & 78161 & 0.5 & 1000 & 16.53 & 6439 & 57 & 86 & 0.21 & $2.2 \mathrm{E}+04$ \\
\hline & 2 & {$\left[\mathrm{C}_{5} \mathrm{H}_{5} \mathrm{ClN}_{3} \mathrm{P}\right]^{-}$} & 172.9935 & 172.9910 & 78161 & 1.5 & 1000 & 16.53 & 6439 & 57 & 86 & 0.21 & $2.0 \mathrm{E}+04$ \\
\hline & 3 & {$\left[\mathrm{C}_{9} \mathrm{H}_{2} \mathrm{ClN}_{2}\right]^{-}$} & 172.9935 & 172.9907 & 78161 & 1.8 & 1000 & 16.53 & 6440 & 56 & 84 & 0.21 & $2.0 \mathrm{E}+04$ \\
\hline
\end{tabular}




\begin{tabular}{|c|c|c|c|c|c|c|c|c|c|c|c|c|c|}
\hline & 4 & {$\left[\mathrm{C}_{6} \mathrm{H}_{6} \mathrm{ClN}_{2} \mathrm{~S}\right]^{-}$} & 172.9935 & 172.9940 & 78161 & 1.7 & 999 & 16.53 & 6440 & 56 & 84 & 0.21 & $1.9 \mathrm{E}+04$ \\
\hline & 5 & {$\left[\mathrm{C}_{6} \mathrm{H}_{4} \mathrm{ClNO}_{3}\right]^{-}$} & 172.9935 & 172.9880 & 78161 & 4.3 & 1000 & 16.53 & 6439 & 56 & 84 & 0.21 & $1.8 \mathrm{E}+04$ \\
\hline & 6 & {$\left[\mathrm{C}_{5} \mathrm{H}_{4} \mathrm{ClN}_{3} \mathrm{O}_{2}\right]^{-}$} & 172.9935 & 172.9992 & 78161 & 6.7 & 1000 & 16.53 & 6442 & 47 & 71 & 0.21 & $1.5 \mathrm{E}+04$ \\
\hline & 7 & {$\left[\mathrm{C}_{7} \mathrm{H}_{6} \mathrm{ClO}_{3}\right]^{-}$} & 172.9935 & 173.0006 & 78161 & 8.0 & 1000 & 16.53 & 6413 & 36 & 56 & 0.22 & $1.1 \mathrm{E}+04$ \\
\hline & 8 & {$\left[\mathrm{C}_{8} \mathrm{H}_{7} \mathrm{Cl}_{2}\right]^{-}$} & 172.9935 & 172.9925 & 78161 & 2.0 & 965 & 16.53 & 6388 & 34 & 59 & 0.24 & $1.0 \mathrm{E}+04$ \\
\hline & 9 & {$\left[\mathrm{C}_{7} \mathrm{H}_{6} \mathrm{ClOS}\right]^{-}$} & 172.9927 & 172.9828 & 67265 & 8.9 & 999 & 16.54 & 3027 & 19 & 42 & 0.15 & $7.6 \mathrm{E}+03$ \\
\hline & 10 & {$\left[\mathrm{C}_{5} \mathrm{H}_{11} \mathrm{Cl}_{2} \mathrm{~S}\right]^{-}$} & 172.9935 & 172.9959 & 78161 & 5.1 & 955 & 16.53 & 6345 & 25 & 43 & 0.24 & $5.5 \mathrm{E}+03$ \\
\hline & & & & & & & & & & & & & \\
\hline \multicolumn{2}{|c|}{$\begin{array}{l}\text { Order of the candidate for } \\
\text { the isotopic feature }\end{array}$} & $\begin{array}{l}\text { Candidate } \\
\text { molecular ion } \\
\text { formula }\end{array}$ & $\begin{array}{c}\text { Detected } \\
\text { mass of } \\
\text { molecular } \\
\text { ion (Da) }\end{array}$ & $\begin{array}{c}\text { Exact mass } \\
\text { of } \\
\text { molecular } \\
\text { ion (Da) }\end{array}$ & Intensity & $\begin{array}{l}\mathrm{NE} \\
\mathrm{ME} \\
(\mathrm{mD} \\
\text { a) }\end{array}$ & $\begin{array}{l}\text { PCS } \\
(\%)\end{array}$ & $\begin{array}{l}\mathrm{RT} \\
(\mathrm{min})\end{array}$ & $\begin{array}{l}\text { Peak } \\
\text { Area }\end{array}$ & $\begin{array}{l}\text { ND } \\
\text { CS }\end{array}$ & $\begin{array}{c}\mathrm{RCS} \\
(\%)\end{array}$ & RPW & $\begin{array}{c}\text { Peak } \\
\text { Identificat } \\
\text { ion score }\end{array}$ \\
\hline \multicolumn{14}{|c|}{ Monochlorodimethylpropylphenol $\left(\mathrm{C}_{11} \mathrm{H}_{15} \mathrm{ClO}\right)$} \\
\hline \multirow{2}{*}{ Isomer 1} & 1 & {$\left[\mathrm{C}_{9} \mathrm{H}_{13} \mathrm{ClN}_{3}\right]^{-}$} & 198.0814 & 198.0799 & 14003 & 2.8 & 953 & 21.99 & 1571 & 27 & 40 & 0.17 & $1.5 \mathrm{E}+03$ \\
\hline & 2 & {$\left[\mathrm{C}_{11} \mathrm{H}_{15} \mathrm{ClO}\right]^{-}$} & 198.0814 & 198.0812 & 14003 & 2.5 & 960 & 21.99 & 1264 & 21 & 47 & 0.31 & $1.1 \mathrm{E}+03$ \\
\hline \multirow[b]{2}{*}{ Isomer 2} & 1 & $\mathrm{ClO}^{-1}$ & 1980813 & 1980812 & 8674 & 61 & 935 & 2253 & 515 & 5 & 30 & 071 & $0,6 \mathrm{~F}+01$ \\
\hline & 2 & {$\left[\mathrm{C}_{9} \mathrm{H}_{13} \mathrm{ClN}_{3}\right]^{-}$} & 198.0819 & 198.0799 & 8191 & 3.3 & 933 & 22.47 & 538 & 5 & 27 & 0.68 & $9.0 \mathrm{E}+01$ \\
\hline & & & & & & & & & & & & & \\
\hline \multirow{5}{*}{ Isomer 3} & 1 & {$\left[\mathrm{C}_{11} \mathrm{H}_{14} \mathrm{ClO}\right]^{-}$} & 197.0762 & 197.0734 & 40052 & 1.8 & 955 & 24.39 & 3421 & 15 & 33 & 0.29 & $1.3 \mathrm{E}+03$ \\
\hline & 2 & {$\left[\mathrm{C}_{9} \mathrm{H}_{12} \mathrm{ClN}_{3}\right]^{-}$} & 197.0762 & 197.0720 & 40052 & 2.8 & 953 & 24.39 & 3361 & 14 & 31 & 0.28 & $1.1 \mathrm{E}+03$ \\
\hline & 3 & {$\left[\mathrm{C}_{10} \mathrm{H}_{14} \mathrm{ClN}_{2}\right]^{-}$} & 197.0762 & 197.0846 & 40052 & 8.3 & 968 & 24.39 & 1367 & 4 & 27 & 0.43 & $5.2 \mathrm{E}+02$ \\
\hline & 4 & {$\left[\mathrm{C}_{11} \mathrm{H}_{15} \mathrm{ClO}\right]^{-}$} & 198.0814 & 198.0812 & 10236 & 2.2 & 941 & 24.32 & 596 & 8 & 44 & 0.71 & $2.0 \mathrm{E}+02$ \\
\hline & 5 & {$\left[\mathrm{C}_{9} \mathrm{H}_{13} \mathrm{ClN}_{3}\right]^{-}$} & 198.0814 & 198.0799 & 10236 & 5.6 & 942 & 24.32 & 624 & 8 & 42 & 0.73 & $1.8 \mathrm{E}+02$ \\
\hline
\end{tabular}


Table S.12. Summary of the IPDC algorithm results for the detected novel contaminants identified in LMLT 2014 (GC $\times$ GC-HRT) ${ }^{9}$ and in LMLT 2016 (Positive APGC-QToF). Additional molecular formula matches are provided for the positive APGC-QToF features. The positive APGC-QToF candidate that matches the molecular formula identified by the $\mathrm{GC} \times \mathrm{GC}-\mathrm{ToF}$ is highlighted in blue.

\begin{tabular}{|c|c|c|c|c|c|c|c|c|c|c|c|c|c|}
\hline \multicolumn{2}{|c|}{$\begin{array}{l}\text { Order of the candidate for } \\
\text { the isotopic feature }\end{array}$} & $\begin{array}{l}\text { Candidate } \\
\text { molecular ion } \\
\text { formula }\end{array}$ & $\begin{array}{l}\text { Detected } \\
\text { mass of } \\
\text { molecular } \\
\text { ion }(\mathrm{Da})\end{array}$ & $\begin{array}{l}\text { Exact mass } \\
\text { of molecular } \\
\text { ion (Da) }\end{array}$ & Intensity & $\begin{array}{l}\text { NEME } \\
(\mathrm{mDa})\end{array}$ & $\begin{array}{l}\text { PCS } \\
(\%)\end{array}$ & $\begin{array}{l}\mathrm{RT} \\
(\mathrm{min})\end{array}$ & $\begin{array}{l}\text { Peak } \\
\text { Area }\end{array}$ & $\begin{array}{l}\text { ND } \\
\text { CS }\end{array}$ & $\begin{array}{l}\text { RCS } \\
(\%)\end{array}$ & RPW & $\begin{array}{l}\text { Peak } \\
\text { Identificati } \\
\text { on score }\end{array}$ \\
\hline \multicolumn{14}{|c|}{$\mathrm{C}_{12} \mathrm{H}_{19} \mathrm{ClO}$} \\
\hline \multirow{3}{*}{ Isomer 1} & 1 & {$\left[\mathrm{C}_{13} \mathrm{H}_{19} \mathrm{Cl}\right]^{+}$} & 210.1222 & 210.1176 & 246351 & 3.9 & 959 & 28.63 & 15806 & 58 & 49 & 0.07 & $1.2 \mathrm{E}+04$ \\
\hline & 2 & {$\left[\mathrm{C}_{11} \mathrm{H}_{19} \mathrm{ClN}_{2}\right]^{+}$} & 214.1223 & 214.1238 & 7420 & 2.0 & 966 & 28.66 & 312 & 9 & 58 & 0.40 & $5.2 \mathrm{E}+02$ \\
\hline & 3 & {$\left[\mathrm{C}_{12} \mathrm{H}_{19} \mathrm{ClO}\right]^{+}$} & 214.1223 & 214.1125 & 3806 & 5.6 & 968 & 28.64 & 174 & 3 & 19 & 0.45 & $1.0 \mathrm{E}+02$ \\
\hline & & & & & & & & & & & & & \\
\hline \multirow{5}{*}{ Isomer 2} & 1 & {$\left[\mathrm{C}_{12} \mathrm{H}_{20} \mathrm{ClN}\right]^{+}$} & 213.1216 & 213.1285 & 6329 & 5.6 & 986 & 31.36 & 288 & 6 & 32 & 0.40 & $4.8 \mathrm{E}+02$ \\
\hline & 2 & {$\left[\mathrm{C}_{12} \mathrm{H}_{20} \mathrm{ClN}\right]^{+}$} & 213.1185 & 213.1285 & 7641 & 8.1 & 971 & 31.41 & 294 & 3 & 26 & 0.65 & $1.6 \mathrm{E}+02$ \\
\hline & 3 & {$\left[\mathrm{C}_{12} \mathrm{H}_{19} \mathrm{ClO}\right]^{+}$} & 214.1210 & 214.1125 & 1525 & 2.8 & 912 & 31.43 & 146 & 6 & 23 & 0.70 & $1.3 \mathrm{E}+01$ \\
\hline & 4 & {$\left[\mathrm{C}_{10} \mathrm{H}_{17} \mathrm{ClN}_{3}\right]^{+}$} & 214.1210 & 214.1112 & 1525 & 3.7 & 912 & 31.43 & 145 & 5 & 19 & 0.69 & $1.1 \mathrm{E}+01$ \\
\hline & 5 & {$\left[\mathrm{C}_{11} \mathrm{H}_{19} \mathrm{ClN}_{2}\right]^{+}$} & 214.1210 & 214.1238 & 1525 & 4.4 & 764 & 31.43 & 71 & 4 & 34 & 0.48 & $0.0 \mathrm{E}+00$ \\
\hline \multicolumn{14}{|c|}{$\mathrm{C}_{9} \mathrm{H}_{11} \mathrm{ClO}_{2}$} \\
\hline \multirow{7}{*}{ Isomer 1} & 1 & {$\left[\mathrm{C}_{9} \mathrm{H}_{11} \mathrm{ClO}_{2}\right]^{+}$} & 186.0478 & 186.0448 & 42144 & 2.9 & 957 & 15.75 & 2487 & 30 & 74 & 0.22 & $2.9 \mathrm{E}+03$ \\
\hline & 2 & {$\left[\mathrm{C}_{8} \mathrm{H}_{11} \mathrm{ClN}_{2} \mathrm{O}\right]^{+}$} & 186.0478 & 186.0560 & 42144 & 6.1 & 957 & 15.75 & 2482 & 29 & 71 & 0.22 & $2.7 \mathrm{E}+03$ \\
\hline & 3 & {$\left[\mathrm{C}_{7} \mathrm{H}_{9} \mathrm{ClN}_{3} \mathrm{O}\right]^{+}$} & 186.0478 & 186.0434 & 42144 & 4.2 & 957 & 15.75 & 2407 & 22 & 58 & 0.23 & $2.1 \mathrm{E}+03$ \\
\hline & 4 & {$\left[\mathrm{C}_{7} \mathrm{H}_{10} \mathrm{ClN}_{3} \mathrm{O}\right]^{+}$} & 187.0510 & 187.0513 & 3001 & 1.5 & 973 & 15.74 & 155 & 14 & 81 & 0.38 & $5.0 \mathrm{E}+02$ \\
\hline & 5 & {$\left[\mathrm{C}_{9} \mathrm{H}_{2} \mathrm{ClO}_{2}\right]^{+}$} & 187.0510 & 187.0526 & 3001 & 2.4 & 976 & 15.74 & 155 & 13 & 76 & 0.38 & $4.9 \mathrm{E}+02$ \\
\hline & 6 & {$\left[\mathrm{C}_{9} \mathrm{H}_{14} \mathrm{ClP}\right]^{+}$} & 188.0524 & 188.0522 & 822 & 2.9 & 962 & 15.75 & 30 & 4 & 65 & 0.43 & $1.4 \mathrm{E}+01$ \\
\hline & 7 & {$\left[\mathrm{C}_{8} \mathrm{H}_{11} \mathrm{ClNO}_{2}\right]^{+}$} & 188.0524 & 188.0479 & 822 & 2.6 & 926 & 15.75 & 30 & 3 & 49 & 0.43 & $4.0 \mathrm{E}+00$ \\
\hline & & & & & & & & & & & & & \\
\hline \multirow{3}{*}{ Isomer 2} & 1 & {$\left[\mathrm{C}_{8} \mathrm{H}_{11} \mathrm{ClN}_{2} \mathrm{O}\right]^{+}$} & 186.0521 & 186.0560 & 21234 & 3.3 & 968 & 37.31 & 1874 & 27 & 61 & 0.35 & $2.2 \mathrm{E}+03$ \\
\hline & 2 & {$\left[\mathrm{C}_{9} \mathrm{H}_{11} \mathrm{ClO}_{2}\right]^{+}$} & 186.0521 & 186.0448 & 21234 & 5.0 & 961 & 37.31 & 1398 & 16 & 44 & 0.26 & $1.3 \mathrm{E}+03$ \\
\hline & 3 & {$\left[\mathrm{C}_{7} \mathrm{H}_{9} \mathrm{ClN}_{3} \mathrm{O}\right]^{+}$} & 186.0530 & 186.0434 & 9690 & 5.5 & 960 & 37.32 & 478 & 7 & 31 & 0.33 & $3.7 \mathrm{E}+02$ \\
\hline \multicolumn{14}{|c|}{$\mathrm{C}_{9} \mathrm{H}_{13} \mathrm{ClO}_{2}$} \\
\hline \multirow{3}{*}{ Isomer 1} & 1 & {$\left[\mathrm{C}_{8} \mathrm{H}_{13} \mathrm{ClN}_{2} \mathrm{O}\right]^{+}$} & 188.0684 & 188.0717 & 21107 & 4.1 & 966 & 16.34 & 1876 & 18 & 72 & 0.73 & $1.2 \mathrm{E}+03$ \\
\hline & 2 & {$\left[\mathrm{C}_{8} \mathrm{H}_{13} \mathrm{ClN}_{2} \mathrm{O}\right]^{+}$} & 188.0677 & 188.0717 & 17859 & 6.0 & 966 & 16.24 & 1419 & 12 & 55 & 0.75 & $7.3 \mathrm{E}+02$ \\
\hline & 3 & {$\left[\mathrm{C}_{9} \mathrm{H}_{13} \mathrm{ClO}_{2}\right]^{+}$} & 188.0684 & 188.0605 & 20594 & 5.5 & 965 & 16.33 & 1829 & 10 & 42 & 0.77 & $6.2 \mathrm{E}+02$ \\
\hline
\end{tabular}




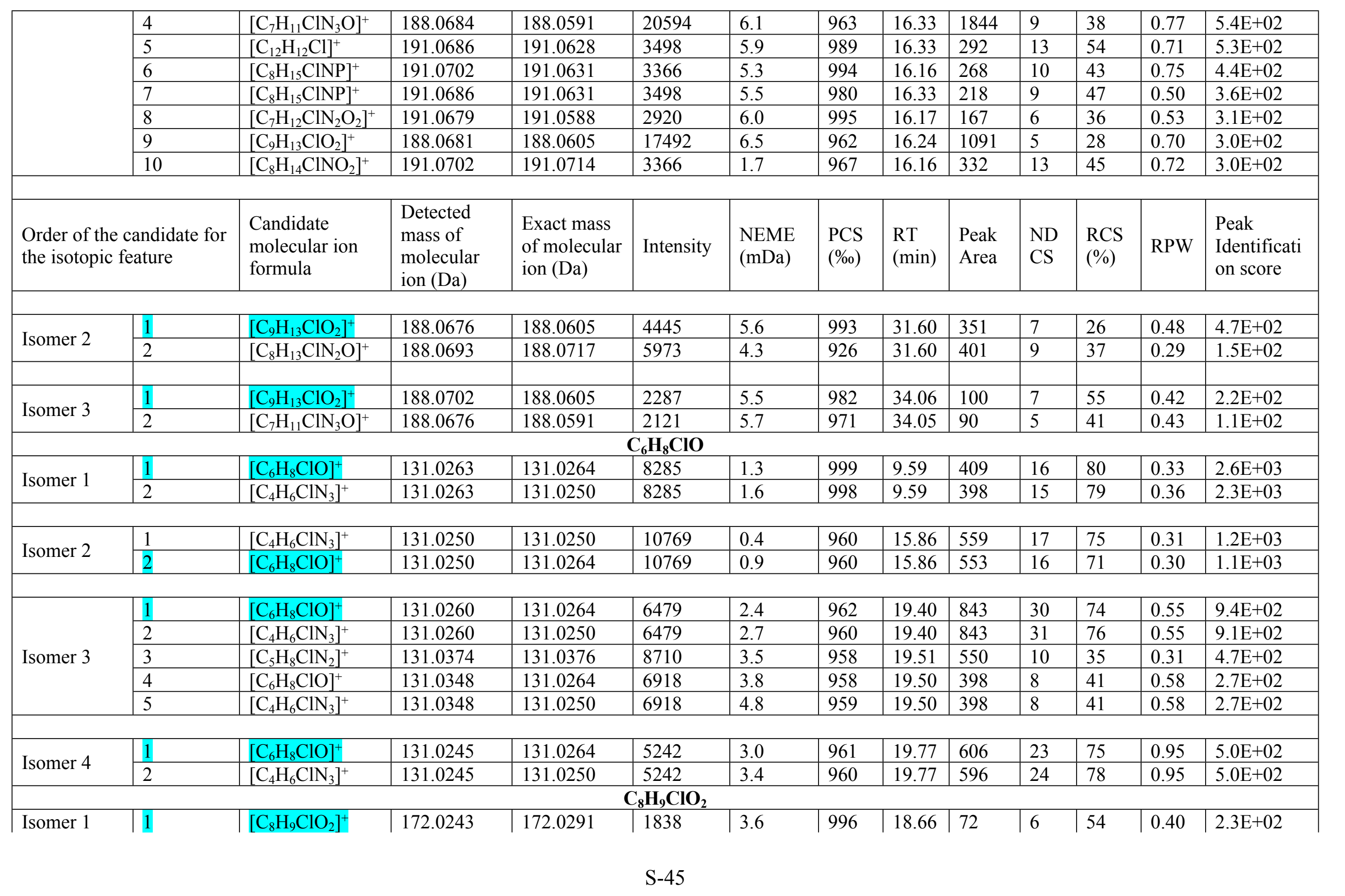




\begin{tabular}{|c|c|c|c|c|c|c|c|c|c|c|c|c|c|}
\hline & 2 & {$\left[\mathrm{C}_{6} \mathrm{H}_{7} \mathrm{ClN}_{3} \mathrm{O}\right]^{+}$} & 172.0243 & 172.0278 & 1838 & 3.0 & 996 & 18.66 & 72 & 6 & 54 & 0.40 & $2.3 \mathrm{E}+02$ \\
\hline & & & & & & & & & & & & & \\
\hline \multirow{6}{*}{ Isomer 2} & 1 & {$\left[\mathrm{C}_{6} \mathrm{H}_{7} \mathrm{ClN}_{3} \mathrm{O}\right]^{+}$} & 172.0249 & 172.0278 & 4539 & 1.8 & 992 & 18.85 & 205 & 12 & 74 & 0.36 & $1.0 \mathrm{E}+03$ \\
\hline & 2 & {$\left[\mathrm{C}_{8} \mathrm{H}_{9} \mathrm{ClO}_{2}\right]^{+}$} & 172.0249 & 172.0291 & 4539 & 2.2 & 994 & 18.85 & 205 & 11 & 68 & 0.37 & $9.7 \mathrm{E}+02$ \\
\hline & 3 & {$\left[\mathrm{C}_{8} \mathrm{H}_{10} \mathrm{ClP}\right]^{+}$} & 172.0249 & \begin{tabular}{|l|}
172.0209 \\
\end{tabular} & 4539 & 6.1 & 995 & 18.85 & 200 & 11 & 70 & 0.35 & $9.6 \mathrm{E}+02$ \\
\hline & 4 & {$\left[\mathrm{C}_{7} \mathrm{H}_{10} \mathrm{ClNP}\right]^{+}$} & 174.0243 & \begin{tabular}{|l|}
174.0240 \\
\end{tabular} & 1136 & 5.6 & 965 & 18.85 & 39 & 4 & 53 & 0.41 & $3.0 \mathrm{E}+01$ \\
\hline & 5 & {$\left[\mathrm{C}_{6} \mathrm{H}_{7} \mathrm{ClN}_{2} \mathrm{O}_{2}\right]^{+}$} & 174.0243 & 174.0196 & 1136 & 5.3 & 973 & 18.85 & 33 & 3 & 46 & 0.46 & $2.5 \mathrm{E}+01$ \\
\hline & 6 & {$\left[\mathrm{C}_{11} \mathrm{H}_{7} \mathrm{Cl}\right]^{+}$} & 174.0243 & 174.0237 & 1136 & 5.4 & 966 & 18.85 & 33 & 3 & 46 & 0.46 & $2.1 \mathrm{E}+01$ \\
\hline & & & & & & & & & & & & & \\
\hline \multicolumn{2}{|c|}{$\begin{array}{l}\text { Order of the candidate for } \\
\text { the isotopic feature }\end{array}$} & $\begin{array}{l}\text { Candidate } \\
\text { molecular ion } \\
\text { formula }\end{array}$ & $\begin{array}{l}\text { Detected } \\
\text { mass of } \\
\text { molecular } \\
\text { ion }(\mathrm{Da}) \\
\end{array}$ & $\begin{array}{l}\text { Exact mass } \\
\text { of molecular } \\
\text { ion }(\mathrm{Da})\end{array}$ & Intensity & $\begin{array}{l}\text { NEME } \\
(\mathrm{mDa})\end{array}$ & $\begin{array}{l}\text { PCS } \\
(\%))\end{array}$ & $\begin{array}{l}\text { RT } \\
(\mathrm{min})\end{array}$ & $\begin{array}{l}\text { Peak } \\
\text { Area }\end{array}$ & $\begin{array}{l}\text { ND } \\
\text { CS }\end{array}$ & $\begin{array}{l}\text { RCS } \\
(\%)\end{array}$ & RPW & $\begin{array}{l}\text { Peak } \\
\text { Identificati } \\
\text { on score }\end{array}$ \\
\hline \multirow{9}{*}{ Isomer 3} & 1 & {$\left[\mathrm{C}_{6} \mathrm{H}_{7} \mathrm{ClN}_{3} \mathrm{O}\right]^{+}$} & 172.0245 & 172.0278 & 24709 & 1.8 & 995 & 22.84 & 1115 & 17 & 84 & 0.33 & $4.2 \mathrm{E}+03$ \\
\hline & 2 & {$\left[\mathrm{C}_{8} \mathrm{H}_{9} \mathrm{ClO}_{2}\right]^{+}$} & 172.0245 & \begin{tabular}{|l|}
172.0291 \\
\end{tabular} & 24709 & 2.8 & 994 & 22.84 & 1115 & 17 & 84 & 0.33 & $4.0 \mathrm{E}+03$ \\
\hline & 3 & {$\left[\mathrm{C}_{8} \mathrm{H}_{10} \mathrm{ClP}\right]^{+}$} & 172.0245 & 172.0209 & 24709 & 4.9 & 994 & 22.84 & 1120 & 14 & 70 & 0.34 & $3.1 \mathrm{E}+03$ \\
\hline & 4 & {$\left[\mathrm{C}_{7} \mathrm{H}_{7} \mathrm{ClNO}_{2}\right]^{+}$} & 172.0245 & 172.0166 & 24709 & 8.9 & 994 & 22.84 & 1148 & 9 & 44 & 0.36 & $1.9 \mathrm{E}+03$ \\
\hline & 5 & {$\left[\mathrm{C}_{11} \mathrm{H}_{7} \mathrm{Cl}\right]^{+}$} & 174.0225 & 174.0237 & 5121 & 1.8 & 964 & 22.84 & 202 & 7 & 48 & 0.36 & $3.2 \mathrm{E}+02$ \\
\hline & 6 & {$\left[\mathrm{C}_{6} \mathrm{H}_{7} \mathrm{ClN}_{2} \mathrm{O}_{2}\right]^{+}$} & 174.0225 & 174.0196 & 5121 & 3.9 & 965 & 22.84 & 176 & 5 & 40 & 0.36 & $2.3 \mathrm{E}+02$ \\
\hline & 7 & {$\left[\mathrm{C}_{7} \mathrm{H}_{10} \mathrm{ClNP}\right]^{+}$} & 174.0225 & \begin{tabular}{|l|}
174.0240 \\
\end{tabular} & 5121 & 2.2 & 961 & 22.84 & 176 & 5 & 40 & 0.36 & \begin{tabular}{|l|}
$2.1 \mathrm{E}+02$ \\
\end{tabular} \\
\hline & 8 & {$\left[\mathrm{C}_{7} \mathrm{H}_{9} \mathrm{ClNO}_{2}\right]^{+}$} & 174.0225 & 174.0322 & 5121 & 6.0 & 960 & 22.84 & 156 & 3 & 28 & 0.42 & $1.1 \mathrm{E}+02$ \\
\hline & 9 & {$\left[\mathrm{C}_{7} \mathrm{H}_{9} \mathrm{ClNS}\right]^{+}$} & 174.0180 & 174.0145 & 1333 & 6.8 & 961 & 22.82 & 52 & 3 & 32 & 0.43 & $2.8 \mathrm{E}+01$ \\
\hline \multirow{4}{*}{ Isomer 4} & 1 & $\mid\left[\mathrm{C}_{6} \mathrm{H}_{7} \mathrm{ClN}_{3} \mathrm{O}^{+}\right.$ & 172.0289 & 172.0278 & 4894 & 1.3 & 972 & 35.34 & 300 & 16 & 74 & 0.42 & $7.6 \mathrm{E}+02$ \\
\hline & 2 & {$\left[\mathrm{C}_{8} \mathrm{H}_{9} \mathrm{ClO}_{2}\right]^{+}$} & 172.0289 & 172.0291 & 4894 & 1.3 & 972 & 35.34 & 306 & 16 & 73 & 0.44 & $7.4 \mathrm{E}+02$ \\
\hline & 3 & {$\left[\mathrm{C}_{8} \mathrm{H}_{10} \mathrm{ClP}\right]^{+}$} & 172.0289 & \begin{tabular}{|l|}
172.0209 \\
\end{tabular} & 4894 & 4.6 & 970 & 35.34 & 257 & 12 & 57 & 0.33 & $5.5 \mathrm{E}+02$ \\
\hline & 4 & {$\left[\mathrm{C}_{7} \mathrm{H}_{9} \mathrm{ClN}_{2} \mathrm{O}\right]^{+}$} & 172.0322 & 172.0404 & 2018 & 3.8 & 975 & 35.38 & 74 & 4 & 38 & 0.45 & $9.4 \mathrm{E}+01$ \\
\hline \multicolumn{14}{|c|}{$\mathrm{C}_{11} \mathrm{H}_{17} \mathrm{ClO}_{2}$} \\
\hline \multirow{3}{*}{ Isomer 1} & 1 & {$\left[\mathrm{C}_{10} \mathrm{H}_{17} \mathrm{ClN}_{2} \mathrm{O}\right]^{+}$} & 216.1016 & 216.1030 & 11849 & 3.9 & 965 & 33.86 & 816 & 16 & 62 & 0.47 & $9.1 \mathrm{E}+02$ \\
\hline & 2 & {$\left[\mathrm{C}_{11} \mathrm{H}_{17} \mathrm{ClO}_{2}\right]^{+}$} & 216.1007 & 216.0918 & 10986 & 4.8 & 971 & 33.87 & 833 & 11 & 39 & 0.46 & $7.1 \mathrm{E}+02$ \\
\hline & 3 & {$\left[\mathrm{C}_{9} \mathrm{H}_{15} \mathrm{ClN}_{3} \mathrm{O}\right]^{+}$} & 216.0993 & 216.0904 & 7995 & 5.4 & 971 & 33.90 & 629 & 8 & 28 & 0.48 & $4.2 \mathrm{E}+02$ \\
\hline & & & & & & & & & & & & & \\
\hline \multirow{3}{*}{ Isomer 2} & 1 & {$\left[\mathrm{C}_{11} \mathrm{H}_{17} \mathrm{ClO}_{2}\right]^{+}$} & 216.1013 & 216.0918 & 7658 & 5.2 & 974 & 34.34 & 353 & 6 & 23 & 0.26 & $4.3 \mathrm{E}+02$ \\
\hline & 2 & {$\left[\mathrm{C}_{10} \mathrm{H}_{17} \mathrm{ClN}_{2} \mathrm{O}\right]^{+}$} & 216.1009 & \begin{tabular}{|l|}
216.1030 \\
\end{tabular} & 8507 & 3.2 & 959 & 34.35 & 462 & 8 & 36 & 0.36 & $4.0 \mathrm{E}+02$ \\
\hline & 3 & {$\left[\mathrm{C}_{9} \mathrm{H}_{15} \mathrm{ClN}_{3} \mathrm{O}\right]^{+}$} & 216.0994 & 216.0904 & 2011 & 5.4 & 974 & \begin{tabular}{|l|}
34.37 \\
\end{tabular} & 137 & 4 & 21 & 0.64 & $7.4 \mathrm{E}+01$ \\
\hline
\end{tabular}




\begin{tabular}{|c|c|c|c|c|c|c|c|c|c|c|c|c|c|}
\hline \multirow{6}{*}{ Isomer 3} & 1 & {$\left[\mathrm{C}_{11} \mathrm{H}_{17} \mathrm{ClNO}^{+}\right.$} & 214.0961 & 214.0999 & 187512 & 4.1 & 958 & 34.63 & 11522 & 35 & 76 & 0.23 & $5.4 \mathrm{E}+03$ \\
\hline & 2 & {$\left[\mathrm{C}_{11} \mathrm{H}_{18} \mathrm{ClNO}\right]^{+}$} & 215.0994 & 215.1078 & 17495 & 5.2 & 980 & 34.62 & 1002 & 14 & 49 & 0.29 & $1.8 \mathrm{E}+03$ \\
\hline & 3 & {$\left[\mathrm{C}_{10} \mathrm{H}_{16} \mathrm{ClN}_{2} \mathrm{O}\right]^{+}$} & 215.1003 & 215.0952 & 20061 & 3.9 & 976 & 34.63 & 1268 & 14 & 36 & 0.27 & $1.6 \mathrm{E}+03$ \\
\hline & 4 & {$\left[\mathrm{C}_{10} \mathrm{H}_{15} \mathrm{ClN}_{2} \mathrm{O}\right]^{+}$} & 214.0961 & 214.0874 & 178498 & 7.4 & 955 & 34.61 & 11510 & 9 & 23 & 0.32 & $1.2 \mathrm{E}+03$ \\
\hline & 5 & {$\left[\mathrm{C}_{11} \mathrm{H}_{17} \mathrm{ClO}_{2}\right]^{+}$} & 216.0984 & 216.0918 & 1386 & 2.1 & 831 & \begin{tabular}{|l|}
34.61 \\
\end{tabular} & 61 & 3 & 28 & 0.45 & $1.0 \mathrm{E}+00$ \\
\hline & 6 & {$\left[\mathrm{C}_{9} \mathrm{H}_{15} \mathrm{ClN}_{3} \mathrm{O}\right]^{+}$} & 216.0984 & 216.0904 & 1386 & 2.8 & 802 & 34.61 & 61 & 3 & 28 & 0.45 & $0.0 \mathrm{E}+00$ \\
\hline \multicolumn{2}{|c|}{$\begin{array}{l}\text { Order of the candidate for } \\
\text { the isotopic feature }\end{array}$} & $\begin{array}{l}\text { Candidate } \\
\text { molecular ion } \\
\text { formula }\end{array}$ & \begin{tabular}{|l} 
Detected \\
mass of \\
molecular \\
ion $(\mathrm{Da})$ \\
\end{tabular} & $\begin{array}{l}\text { Exact mass } \\
\text { of molecular } \\
\text { ion }(\mathrm{Da})\end{array}$ & Intensity & $\begin{array}{l}\text { NEME } \\
(\mathrm{mDa})\end{array}$ & $\begin{array}{l}\text { PCS } \\
(\%)\end{array}$ & $\begin{array}{l}\mathrm{RT} \\
(\mathrm{min})\end{array}$ & $\begin{array}{l}\text { Peak } \\
\text { Area }\end{array}$ & $\begin{array}{l}\text { ND } \\
\text { CS }\end{array}$ & $\begin{array}{l}\mathrm{RCS} \\
(\%)\end{array}$ & RPW & $\begin{array}{l}\text { Peak } \\
\text { Identificati } \\
\text { on score }\end{array}$ \\
\hline \multicolumn{14}{|c|}{$\mathrm{C}_{7} \mathrm{H}_{6} \mathrm{BrClO}_{2}$} \\
\hline \multirow{10}{*}{ Isomer 1} & 1 & {$\left[\mathrm{C}_{5} \mathrm{H}_{7} \mathrm{Cl}_{3} \mathrm{O}_{2} \mathrm{~S}\right]^{+}$} & 237.9227 & 237.9203 & 1836 & 3.1 & 983 & 23.94 & 62 & 5 & 54 & 0.43 & $5.6 \mathrm{E}+02$ \\
\hline & 2 & {$\left[\mathrm{C}_{4} \mathrm{H}_{5} \mathrm{Cl}_{3} \mathrm{NO}_{4}\right]^{+}$} & 235.9264 & 235.9284 & 1489 & 1.9 & 981 & 23.93 & 59 & 6 & 59 & 0.46 & $5.0 \mathrm{E}+02$ \\
\hline & 3 & {$\left[\mathrm{C}_{5} \mathrm{H}_{8} \mathrm{Cl}_{3} \mathrm{O}_{2} \mathrm{P}\right]^{+}$} & 235.9264 & 235.9328 & 1489 & 3.0 & 981 & 23.93 & 59 & 6 & 59 & 0.46 & $4.8 \mathrm{E}+02$ \\
\hline & 4 & {$\left[\mathrm{C}_{7} \mathrm{H}_{3} \mathrm{Cl}_{3} \mathrm{~N}_{2} \mathrm{O}\right]^{+}$} & 235.9264 & 235.9311 & 1489 & 3.0 & 980 & 23.93 & 59 & 6 & 59 & 0.46 & $4.6 \mathrm{E}+02$ \\
\hline & 5 & {$\left[\mathrm{C}_{5} \mathrm{H}_{4} \mathrm{BrClN}_{3} \mathrm{O}\right]^{+}$} & 237.9227 & 237.9204 & 1836 & 2.8 & 992 & 23.94 & 63 & 6 & 63 & 0.44 & $4.6 \mathrm{E}+02$ \\
\hline & 6 & {$\left[\mathrm{C}_{8} \mathrm{H}_{3} \mathrm{Cl}_{3} \mathrm{O}_{2}\right]^{+}$} & 235.9264 & 235.9199 & 1489 & 4.8 & 987 & 23.93 & 52 & 5 & 56 & 0.44 & $4.5 \mathrm{E}+02$ \\
\hline & 7 & {$\left[\mathrm{C}_{7} \mathrm{H}_{6} \mathrm{BrClO}_{2}\right]^{+}$} & 237.9227 & 237.9217 & 1836 & 1.9 & 995 & 23.94 & 62 & 5 & 54 & 0.43 & $4.3 \mathrm{E}+02$ \\
\hline & 8 & {$\left[\mathrm{C}_{4} \mathrm{H}_{6} \mathrm{Cl}_{3} \mathrm{NO}_{2} \mathrm{P}\right]^{+}$} & 235.9264 & 235.9202 & 1489 & 4.7 & 982 & 23.93 & 52 & 5 & 56 & 0.44 & $4.0 \mathrm{E}+02$ \\
\hline & 9 & {$\left[\mathrm{C}_{9} \mathrm{H}_{4} \mathrm{BrNP}\right]^{+}$} & 235.9264 & 235.9265 & 1489 & 1.2 & 989 & 23.93 & 58 & 7 & 70 & 0.45 & $3.9 \mathrm{E}+02$ \\
\hline & 10 & {$\left[\mathrm{C}_{10} \mathrm{H}_{5} \mathrm{BrS}\right]^{+}$} & 235.9264 & 235.9296 & 1489 & 2.3 & 990 & 23.93 & 58 & 7 & 70 & 0.45 & $3.8 \mathrm{E}+02$ \\
\hline \multicolumn{14}{|c|}{ 1,2,4,5-tetrachloro-3,6-dimethoxybenzene $\left(\mathrm{C}_{8} \mathrm{H}_{6} \mathrm{Cl}_{4} \mathrm{O}_{2}\right)$} \\
\hline \multirow{10}{*}{ Isomer 1} & 1 & {$\left[\mathrm{C}_{8} \mathrm{H}_{6} \mathrm{Cl}_{4} \mathrm{O}_{2}\right]^{+}$} & 275.9089 & 275.9093 & 8594 & 2.3 & 988 & 28.20 & 380 & 15 & 85 & 0.37 & $7.2 \mathrm{E}+03$ \\
\hline & 2 & {$\left[\mathrm{C}_{4} \mathrm{H}_{9} \mathrm{Cl}_{4} \mathrm{NO}_{2} \mathrm{P}\right]^{+}$} & 275.9089 & 275.9096 & 8594 & 2.1 & 985 & 28.20 & 380 & 15 & 85 & 0.37 & $6.7 \mathrm{E}+03$ \\
\hline & 3 & {$\left[\mathrm{C}_{5} \mathrm{H}_{7} \mathrm{BrCl}_{2} \mathrm{~N}_{3} \mathrm{O}\right]^{+}$} & 275.9107 & 275.9126 & 7899 & 1.5 & 990 & 28.19 & 354 & 13 & 74 & 0.37 & $6.6 \mathrm{E}+03$ \\
\hline & 4 & {$\left[\mathrm{C}_{4} \mathrm{H}_{9} \mathrm{Cl}_{3} \mathrm{O}_{3} \mathrm{~S}_{2}\right]^{+}$} & 275.9089 & 275.9029 & 8594 & 7.3 & 978 & 28.20 & 375 & 11 & 65 & 0.38 & $6.3 \mathrm{E}+03$ \\
\hline & 5 & {$\left[\mathrm{C}_{6} \mathrm{H}_{4} \mathrm{Cl}_{4} \mathrm{~N}_{3} \mathrm{O}\right]^{+}$} & 275.9089 & 275.9079 & 8594 & 3.3 & 988 & 28.20 & 375 & 13 & 76 & 0.38 & $6.1 \mathrm{E}+03$ \\
\hline & 6 & {$\left[\mathrm{C}_{5} \mathrm{H}_{10} \mathrm{Cl}_{4} \mathrm{O}_{2} \mathrm{~S}\right]^{+}$} & 275.9107 & 275.9126 & 7899 & 1.5 & 984 & 28.19 & 354 & 13 & 74 & 0.37 & $5.5 \mathrm{E}+03$ \\
\hline & 7 & {$\left[\mathrm{C}_{5} \mathrm{H}_{11} \mathrm{Cl}_{4} \mathrm{PS}\right]^{+}$} & 275.9089 & 275.9044 & 8594 & 5.8 & 986 & 28.20 & 375 & 12 & 70 & 0.38 & $5.1 \mathrm{E}+03$ \\
\hline & 8 & {$\left[\mathrm{C}_{4} \mathrm{H}_{8} \mathrm{Cl}_{4} \mathrm{NO}_{4}\right]^{+}$} & 275.9107 & \begin{tabular}{|l|}
275.9178 \\
\end{tabular} & 7899 & 5.5 & 984 & 28.19 & 346 & 12 & 70 & 0.38 & $4.6 \mathrm{E}+03$ \\
\hline & 9 & {$\left[\mathrm{C}_{7} \mathrm{H}_{7} \mathrm{Cl}_{3} \mathrm{NS}_{2}\right]^{+}$} & 275.9089 & 275.9056 & 8594 & 5.6 & 980 & 28.20 & 375 & 12 & 70 & 0.38 & $4.4 \mathrm{E}+03$ \\
\hline & 10 & {$\left[\mathrm{C}_{12} \mathrm{H}_{2} \mathrm{BrClN}\right]^{+}$} & 275.9107 & 275.9037 & 7899 & 7.4 & 992 & 28.19 & 344 & 9 & 54 & 0.40 & $4.3 \mathrm{E}+03$ \\
\hline \multicolumn{14}{|c|}{ Chlorophenyl-pyrimidine $\left(\mathrm{C}_{10} \mathrm{H}_{7} \mathrm{ClN}_{2}\right)$} \\
\hline Isomer 1 & 1 & {$\left[\mathrm{C}_{8} \mathrm{H}_{12} \mathrm{ClOP}\right]^{+}$} & 190.0318 & 190.0315 & 4338 & 0.9 & 970 & 26.72 & 185 & 10 & 69 & 0.41 & $4.7 \mathrm{E}+02$ \\
\hline
\end{tabular}




\begin{tabular}{|c|c|c|c|c|c|c|c|c|c|c|c|c|c|}
\hline & 2 & {$\left[\mathrm{C}_{10} \mathrm{H}_{7} \mathrm{ClN}_{2}\right]^{+}$} & 190.0318 & 190.0298 & 4338 & 2.0 & 970 & 26.72 & 185 & 10 & 69 & 0.41 & $4.3 \mathrm{E}+02$ \\
\hline & 3 & {$\left[\mathrm{C}_{6} \mathrm{H}_{10} \mathrm{ClN}_{3} \mathrm{P}\right]^{+}$} & 190.0318 & 190.0301 & 4338 & 1.8 & 969 & 26.72 & 185 & 10 & 69 & 0.41 & $4.3 \mathrm{E}+02$ \\
\hline & 4 & {$\left[\mathrm{C}_{7} \mathrm{H}_{9} \mathrm{ClNO}_{3}\right]^{+}$} & 190.0318 & 190.0271 & 4338 & 2.9 & 968 & 26.72 & 185 & 10 & 69 & 0.41 & $4.1 \mathrm{E}+02$ \\
\hline & 5 & {$\left[\mathrm{C}_{6} \mathrm{H}_{9} \mathrm{ClN}_{3} \mathrm{O}_{2}\right]^{+}$} & 190.0318 & \begin{tabular}{|l|}
190.0384 \\
\end{tabular} & 4338 & 4.4 & 969 & 26.72 & 188 & 9 & 61 & 0.42 & $3.5 \mathrm{E}+02$ \\
\hline & 6 & {$\left[\mathrm{C}_{8} \mathrm{H}_{11} \mathrm{ClO}_{3}\right]^{+}$} & 190.0318 & \begin{tabular}{|l|}
190.0397 \\
\end{tabular} & 4338 & 5.0 & 971 & 26.72 & 190 & 7 & 47 & 0.43 & $2.9 \mathrm{E}+02$ \\
\hline & \begin{tabular}{|l|}
7 \\
\end{tabular} & {$\left[\mathrm{C}_{7} \mathrm{H}_{11} \mathrm{ClN}_{2} \mathrm{~S}\right]^{+}$} & 190.0309 & 190.0332 & 4211 & 1.2 & 959 & 26.73 & 171 & 7 & 50 & 0.41 & $2.4 \mathrm{E}+02$ \\
\hline & 8 & {$\left[\mathrm{C}_{8} \mathrm{H}_{11} \mathrm{ClOS}\right]^{+}$} & 190.0309 & \begin{tabular}{|l|}
190.0220 \\
\end{tabular} & 4211 & 6.1 & 956 & 26.73 & 164 & 5 & 37 & 0.43 & $1.3 \mathrm{E}+02$ \\
\hline & & & & & & & & & & & & & \\
\hline \multicolumn{2}{|c|}{$\begin{array}{l}\text { Order of the candidate } \\
\text { for the isotopic feature }\end{array}$} & $\begin{array}{l}\text { Candidate } \\
\text { molecular ion } \\
\text { formula }\end{array}$ & $\begin{array}{l}\text { Detected } \\
\text { mass of } \\
\text { molecular } \\
\text { ion (Da) }\end{array}$ & $\begin{array}{l}\text { Exact mass } \\
\text { of molecular } \\
\text { ion }(\mathrm{Da})\end{array}$ & Intensity & $\begin{array}{l}\text { NEME } \\
(\mathrm{mDa})\end{array}$ & $\begin{array}{l}\text { PCS } \\
(\%)\end{array}$ & $\begin{array}{l}\mathrm{RT} \\
(\mathrm{min})\end{array}$ & $\begin{array}{l}\text { Peak } \\
\text { Area }\end{array}$ & $\begin{array}{l}\text { ND } \\
\text { CS }\end{array}$ & $\begin{array}{l}\mathrm{RCS} \\
(\%)\end{array}$ & RPW & $\begin{array}{l}\text { Peak } \\
\text { Identificati } \\
\text { on score }\end{array}$ \\
\hline \multirow{10}{*}{ Isomer 2} & 1 & {$\left[\mathrm{C}_{7} \mathrm{H}_{11} \mathrm{ClN}_{2} \mathrm{P}\right]^{+}$} & 189.0332 & \begin{tabular}{|l}
189.0349 \\
\end{tabular} & 62757 & 1.3 & 957 & 28.88 & 3430 & 23 & 76 & 0.30 & $2.8 \mathrm{E}+03$ \\
\hline & \begin{tabular}{|l|}
2 \\
\end{tabular} & {$\left[\mathrm{C}_{8} \mathrm{H}_{10} \mathrm{ClO}_{3}\right]^{+}$} & 189.0332 & \begin{tabular}{|l|}
189.0319 \\
\end{tabular} & 62757 & 1.4 & 957 & 28.88 & 3430 & 23 & 76 & 0.30 & $2.7 \mathrm{E}+03$ \\
\hline & 3 & {$\left[\mathrm{C}_{11} \mathrm{H}_{8} \mathrm{ClN}\right]^{+}$} & 189.0332 & 189.0346 & 62757 & 1.1 & 956 & 28.88 & 3402 & 22 & 81 & 0.32 & $2.6 \mathrm{E}+03$ \\
\hline & 4 & {$\left[\mathrm{C}_{6} \mathrm{H}_{8} \mathrm{ClN}_{3} \mathrm{O}_{2}\right]^{+}$} & 189.0332 & \begin{tabular}{|l|}
189.0305 \\
\end{tabular} & 62757 & 2.4 & 957 & 28.88 & 3402 & 22 & 81 & 0.32 & $2.5 \mathrm{E}+03$ \\
\hline & \begin{tabular}{|l|}
5 \\
\end{tabular} & {$\left[\mathrm{C}_{7} \mathrm{H}_{10} \mathrm{ClN}_{2} \mathrm{O}_{2}\right]^{+}$} & 189.0332 & \begin{tabular}{|l|}
189.0431 \\
\end{tabular} & 62757 & 7.1 & 956 & 28.88 & 3159 & 12 & 40 & 0.26 & $1.4 \mathrm{E}+03$ \\
\hline & 6 & {$\left[\mathrm{C}_{11} \mathrm{H}_{9} \mathrm{ClN}\right]^{+}$} & 190.0348 & 190.0424 & 4616 & 3.8 & 970 & 28.88 & 195 & 11 & 76 & 0.42 & $4.7 \mathrm{E}+02$ \\
\hline & 7 & {$\left[\mathrm{C}_{8} \mathrm{H}_{11} \mathrm{ClOP}\right]^{+}$} & 189.0333 & 189.0237 & 43280 & 7.2 & 956 & 28.88 & 2163 & 5 & 24 & 0.45 & $4.4 \mathrm{E}+02$ \\
\hline & \begin{tabular}{|l|}
8 \\
\end{tabular} & {$\left[\mathrm{C}_{10} \mathrm{H}_{7} \mathrm{ClN}_{2}\right]^{+}$} & 190.0348 & \begin{tabular}{|l|}
190.0298 \\
\end{tabular} & 4616 & 2.7 & 964 & 28.88 & 217 & 8 & 49 & 0.41 & $3.1 \mathrm{E}+02$ \\
\hline & 9 & {$\left[\mathrm{C}_{8} \mathrm{H}_{11} \mathrm{ClO}_{3}\right]^{+}$} & 190.0345 & \begin{tabular}{|l|}
190.0397 \\
\end{tabular} & 3141 & 3.6 & 969 & 28.88 & 141 & 8 & 58 & 0.47 & $2.4 \mathrm{E}+02$ \\
\hline & 10 & {$\left[\mathrm{C}_{6} \mathrm{H}_{9} \mathrm{ClN}_{3} \mathrm{O}_{2}\right]^{+}$} & 190.0345 & 190.0384 & 3141 & 3.2 & 964 & 28.88 & 152 & 9 & 61 & 0.48 & $2.3 \mathrm{E}+02$ \\
\hline \multicolumn{14}{|c|}{$\mathrm{C}_{10} \mathrm{H}_{4} \mathrm{Cl}_{7} \mathrm{O}$} \\
\hline \multirow{10}{*}{ Isomer 1} & 1 & {$\left[\mathrm{C}_{6} \mathrm{H}_{7} \mathrm{Cl}_{7} \mathrm{NOP}\right]^{+}$} & 386.8086 & 386.8056 & 3231 & 3.0 & 990 & 34.89 & 113 & 6 & 53 & 0.42 & $4.0 \mathrm{E}+03$ \\
\hline & 2 & {$\left[\mathrm{C}_{10} \mathrm{H}_{4} \mathrm{Cl}_{7} \mathrm{O}\right]^{+}$} & 386.8086 & 386.8052 & 3231 & 2.9 & 990 & 34.89 & 113 & 6 & 53 & 0.42 & $3.9 \mathrm{E}+03$ \\
\hline & \begin{tabular}{|l|}
3 \\
\end{tabular} & {$\left[\mathrm{C}_{6} \mathrm{H}_{8} \mathrm{Br}_{2} \mathrm{Cl}_{3} \mathrm{~N}_{3}\right]^{+}$} & 388.8026 & 388.8103 & 2527 & 3.0 & 997 & 34.89 & 96 & 6 & 52 & 0.42 & $3.8 \mathrm{E}+03$ \\
\hline & \begin{tabular}{|l}
4 \\
\end{tabular} & {$\left[\mathrm{C}_{6} \mathrm{H}_{12} \mathrm{Br}_{3} \mathrm{O}_{2} \mathrm{~S}\right]^{+}$} & 386.8086 & 386.8088 & 3231 & 1.9 & 995 & \begin{tabular}{|l|}
34.89 \\
\end{tabular} & 147 & 8 & 54 & 0.44 & $3.8 \mathrm{E}+03$ \\
\hline & 5 & {$\left[\mathrm{C}_{9} \mathrm{H}_{7} \mathrm{BrCl}_{5} \mathrm{O}\right]^{+}$} & 388.8026 & \begin{tabular}{|l|}
388.8070 \\
\end{tabular} & 2527 & 2.3 & 994 & 34.89 & 89 & 6 & 57 & 0.39 & $3.7 \mathrm{E}+03$ \\
\hline & \begin{tabular}{|l}
6 \\
\end{tabular} & {$\left[\mathrm{C}_{5} \mathrm{H}_{11} \mathrm{Br}_{3} \mathrm{NO}_{2} \mathrm{P}\right]^{+}$} & 386.8086 & 386.8058 & 3231 & 2.2 & 995 & \begin{tabular}{|l|}
34.89 \\
\end{tabular} & 127 & 7 & 56 & 0.42 & $3.5 \mathrm{E}+03$ \\
\hline & \begin{tabular}{|l|}
7 \\
\end{tabular} & {$\left[\mathrm{C}_{7} \mathrm{H}_{6} \mathrm{Br}_{3} \mathrm{~N}_{3} \mathrm{O}\right]^{+}$} & 386.8086 & 386.8041 & 3231 & 3.3 & 996 & 34.89 & 127 & 7 & 56 & 0.42 & $3.4 \mathrm{E}+03$ \\
\hline & \begin{tabular}{|l|}
8 \\
\end{tabular} & {$\left[\mathrm{C}_{5} \mathrm{H}_{6} \mathrm{Cl}_{7} \mathrm{~N}_{3} \mathrm{~S}\right]^{+}$} & 386.8086 & \begin{tabular}{|l|}
386.8073 \\
\end{tabular} & 3231 & 2.1 & 990 & 34.89 & 112 & 5 & 45 & 0.41 & $3.4 \mathrm{E}+03$ \\
\hline & 9 & {$\left[\mathrm{C}_{7} \mathrm{H}_{8} \mathrm{Cl}_{7} \mathrm{OS}\right]^{+}$} & 386.8086 & 386.8086 & 3231 & 2.2 & 989 & 34.89 & 112 & 5 & 45 & 0.41 & $3.4 \mathrm{E}+03$ \\
\hline & 10 & {$\left[\mathrm{C}_{8} \mathrm{H}_{2} \mathrm{Cl}_{7} \mathrm{~N}_{3}\right]^{+}$} & 386.8086 & 386.8039 & 3231 & 3.0 & 989 & 34.89 & 113 & 5 & 44 & 0.42 & $3.3 \mathrm{E}+03$ \\
\hline \multicolumn{14}{|c|}{$\mathrm{C}_{14} \mathrm{H}_{13} \mathrm{ClO}_{4}$} \\
\hline Isomer 1 & 1 & $\overline{\left.\mathrm{C}_{17} \mathrm{H}_{11} \mathrm{ClO}_{2}\right]^{+}}$ & 282.0485 & 282.0448 & 81596 & 4.7 & 957 & 37.87 & 4235 & 26 & 60 & 0.18 & $3.4 \mathrm{E}+03$ \\
\hline
\end{tabular}




\begin{tabular}{|c|c|c|c|c|c|c|c|c|c|c|c|c|c|}
\hline & 2 & {$\left[\mathrm{C}_{13} \mathrm{H}_{13} \mathrm{ClNO}_{4}\right]^{+}$} & 282.0485 & 282.0534 & 81596 & 3.3 & 955 & 37.87 & 4619 & 29 & 42 & 0.14 & $3.4 \mathrm{E}+03$ \\
\hline & 3 & {$\left[\mathrm{C}_{17} \mathrm{H}_{11} \mathrm{ClNO}\right]^{+}$} & 280.0503 & 280.0530 & 228428 & 1.9 & 973 & 37.87 & 10933 & 12 & 56 & 0.46 & $3.2 \mathrm{E}+03$ \\
\hline & 4 & {$\left[\mathrm{C}_{16} \mathrm{H}_{11} \mathrm{ClN}_{2} \mathrm{O}\right]^{+}$} & 282.0485 & 282.0560 & 81596 & 5.4 & 957 & 37.87 & 4425 & 25 & 55 & 0.19 & $3.2 \mathrm{E}+03$ \\
\hline & 5 & {$\left[\mathrm{C}_{13} \mathrm{H}_{14} \mathrm{ClNO}_{2} \mathrm{P}\right]^{+}$} & 282.0485 & 282.0451 & 81596 & 4.5 & 954 & \begin{tabular}{|l|}
37.87 \\
\end{tabular} & 4237 & 24 & 53 & 0.18 & $2.9 \mathrm{E}+03$ \\
\hline & 6 & {$\left[\mathrm{C}_{14} \mathrm{H}_{13} \mathrm{ClO}_{4}\right]^{+}$} & 280.0503 & 280.0503 & 228428 & 1.0 & 966 & 37.87 & 10933 & 12 & 56 & 0.46 & $2.8 \mathrm{E}+03$ \\
\hline & 7 & {$\left[\mathrm{C}_{12} \mathrm{H}_{14} \mathrm{ClN}_{3} \mathrm{OP}\right]^{+}$} & 282.0485 & 282.0564 & 81596 & 5.6 & 954 & 37.87 & 4418 & 23 & 51 & 0.19 & $2.6 \mathrm{E}+03$ \\
\hline & 8 & {$\left[\mathrm{C}_{14} \mathrm{H}_{15} \mathrm{ClNOS}^{+}\right.$} & 280.0503 & 280.0564 & 228428 & 5.3 & 965 & 37.87 & 10933 & 12 & 56 & 0.46 & $2.4 \mathrm{E}+03$ \\
\hline & 9 & {$\left[\mathrm{C}_{14} \mathrm{H}_{14} \mathrm{ClO}_{2} \mathrm{P}\right]^{+}$} & 280.0503 & 280.0421 & 228428 & 9.1 & 966 & 37.87 & 10933 & 12 & 56 & 0.46 & $2.3 \mathrm{E}+03$ \\
\hline & 10 & {$\left[\mathrm{C}_{13} \mathrm{H}_{14} \mathrm{ClN}_{2} \mathrm{OP}\right]^{+}$} & 280.0503 & 280.0533 & 228428 & 2.2 & 964 & 37.87 & 10624 & 11 & 53 & 0.46 & $2.3 \mathrm{E}+03$ \\
\hline & & & & & & & & & & & & & \\
\hline \multicolumn{2}{|c|}{$\begin{array}{l}\text { Order of the candidate } \\
\text { for the isotopic feature }\end{array}$} & $\begin{array}{l}\text { Candidate } \\
\text { molecular ion } \\
\text { formula }\end{array}$ & $\begin{array}{l}\text { Detected } \\
\text { mass of } \\
\text { molecular } \\
\text { ion (Da) }\end{array}$ & $\begin{array}{l}\text { Exact mass } \\
\text { of molecular } \\
\text { ion (Da) }\end{array}$ & Intensity & $\begin{array}{l}\text { NEME } \\
(\mathrm{mDa})\end{array}$ & $\begin{array}{l}\text { PCS } \\
(\%))\end{array}$ & $\begin{array}{l}\text { RT } \\
(\min )\end{array}$ & $\begin{array}{l}\text { Peak } \\
\text { Area }\end{array}$ & $\begin{array}{l}\text { ND } \\
\text { CS }\end{array}$ & $\begin{array}{l}\text { RCS } \\
(\%)\end{array}$ & RPW & $\begin{array}{l}\text { Peak } \\
\text { Identificati } \\
\text { on score }\end{array}$ \\
\hline \multirow{10}{*}{ Isomer 2} & 1 & {$\left[\mathrm{C}_{16} \mathrm{H}_{11} \mathrm{ClN}_{2} \mathrm{O}\right]^{+}$} & 282.0485 & 282.0560 & 17278 & 4.7 & 960 & 39.03 & 1392 & 34 & 53 & 0.14 & $2.6 \mathrm{E}+03$ \\
\hline & 2 & {$\left[\mathrm{C}_{13} \mathrm{H}_{13} \mathrm{ClNO}_{4}\right]^{+}$} & 282.0484 & 282.0534 & 17756 & 2.9 & 954 & 39.02 & 1232 & 25 & 47 & 0.13 & $1.9 \mathrm{E}+03$ \\
\hline & 3 & {$\left[\mathrm{C}_{12} \mathrm{H}_{14} \mathrm{ClN}_{3} \mathrm{OP}\right]^{+}$} & 282.0485 & 282.0564 & 17278 & 5.3 & 955 & 39.03 & 1171 & 21 & 41 & 0.18 & $1.3 \mathrm{E}+03$ \\
\hline & 4 & {$\left[\mathrm{C}_{17} \mathrm{H}_{11} \mathrm{ClO}_{2}\right]^{+}$} & 282.0484 & 282.0448 & 17756 & 3.4 & 959 & 39.02 & 981 & 15 & 48 & 0.24 & $1.2 \mathrm{E}+03$ \\
\hline & 5 & {$\left[\mathrm{C}_{9} \mathrm{H}_{16} \mathrm{ClN}_{2} \mathrm{O}_{4} \mathrm{P}\right]^{+}$} & 282.0484 & 282.0537 & 17756 & 3.6 & 948 & 39.02 & 1220 & 20 & 39 & 0.18 & $1.1 \mathrm{E}+03$ \\
\hline & 6 & {$\left[\mathrm{C}_{15} \mathrm{H}_{9} \mathrm{ClN}_{3} \mathrm{O}\right]^{+}$} & 282.0484 & 282.0434 & 17756 & 4.5 & 958 & 39.02 & 881 & 14 & 56 & 0.26 & $1.1 \mathrm{E}+03$ \\
\hline & 7 & {$\left[\mathrm{C}_{13} \mathrm{H}_{14} \mathrm{ClNO}_{2} \mathrm{P}\right]^{+}$} & 282.0484 & 282.0451 & 17756 & 3.5 & 956 & 39.02 & 831 & 12 & 52 & 0.27 & $8.8 \mathrm{E}+02$ \\
\hline & 8 & {$\left[\mathrm{C}_{10} \mathrm{H}_{16} \mathrm{ClO}_{5} \mathrm{P}\right]^{+}$} & 282.0484 & 282.0425 & 17756 & 5.5 & 952 & 39.02 & 864 & 11 & 47 & 0.29 & $6.8 \mathrm{E}+02$ \\
\hline & 9 & {$\left[\mathrm{C}_{12} \mathrm{H}_{11} \mathrm{ClN}_{2} \mathrm{O}_{4}\right]^{+}$} & 282.0484 & 282.0408 & 17756 & 7.0 & 955 & 39.02 & 789 & 10 & 55 & 0.42 & $6.0 \mathrm{E}+02$ \\
\hline & 10 & {$\left[\mathrm{C}_{14} \mathrm{H}_{13} \mathrm{ClO}_{4}\right]^{+}$} & 280.0507 & 280.0503 & 57432 & 1.2 & 927 & 39.03 & 1977 & 7 & 45 & 0.41 & $3.9 \mathrm{E}+02$ \\
\hline \multicolumn{14}{|c|}{ 3,6-dichloro-9H-carbazole $\left(\mathrm{C}_{12} \mathrm{H}_{7} \mathrm{Cl}_{2} \mathrm{~N}\right)$} \\
\hline \multirow{10}{*}{ Isomer 1} & 1 & {$\left[\mathrm{C}_{12} \mathrm{H}_{7} \mathrm{Cl}_{2} \mathrm{~N}\right]^{+}$} & 234.9927 & 234.9956 & 960 & 3.2 & 967 & 40.51 & 58 & 6 & 48 & 0.54 & $8.2 \mathrm{E}+01$ \\
\hline & 2 & {$\left[\mathrm{C}_{8} \mathrm{H}_{10} \mathrm{Cl}_{2} \mathrm{~N}_{2} \mathrm{P}\right]^{+}$} & 234.9927 & 234.9959 & 960 & 2.9 & 958 & 40.51 & 60 & 5 & 38 & 0.55 & $5.3 \mathrm{E}+01$ \\
\hline & 3 & {$\left[\mathrm{C}_{9} \mathrm{H}_{9} \mathrm{Cl}_{2} \mathrm{O}_{3}\right]^{+}$} & 234.9927 & 234.9929 & 960 & 4.5 & 954 & 40.51 & 36 & 4 & 53 & 0.46 & $3.6 \mathrm{E}+01$ \\
\hline & 4 & {$\left[\mathrm{C}_{6} \mathrm{H}_{13} \mathrm{Cl}_{2} \mathrm{O}_{3} \mathrm{~S}\right]^{+}$} & 234.9927 & 234.9963 & 960 & 3.1 & 951 & 40.51 & 49 & 4 & 37 & 0.53 & $3.4 \mathrm{E}+01$ \\
\hline & 5 & {$\left[\mathrm{C}_{7} \mathrm{H}_{7} \mathrm{Cl}_{2} \mathrm{~N}_{3} \mathrm{O}_{2}\right]^{+}$} & 234.9927 & 234.9915 & 960 & 5.4 & 947 & 40.51 & 35 & 3 & 41 & 0.44 & $2.2 \mathrm{E}+01$ \\
\hline & 6 & {$\left[\mathrm{C}_{11} \mathrm{H}_{7} \mathrm{ClNOP}\right]^{+}$} & 234.9981 & 234.9954 & 1020 & 2.6 & 933 & 40.49 & 46 & 4 & 42 & 0.47 & $1.1 \mathrm{E}+01$ \\
\hline & 7 & {$\left[\mathrm{C}_{8} \mathrm{H}_{12} \mathrm{BrO}_{3}\right]^{+}$} & 234.9927 & 234.9970 & 960 & 2.9 & 908 & 40.51 & 42 & 3 & 33 & 0.52 & $7.0 \mathrm{E}+00$ \\
\hline & 8 & {$\left[\mathrm{C}_{7} \mathrm{H}_{8} \mathrm{ClN}_{2} \mathrm{O}_{3} \mathrm{~S}^{+}\right.$} & 234.9927 & 234.9944 & 960 & 2.5 & 935 & 40.51 & 35 & 3 & 41 & \begin{tabular}{|l|}
0.47 \\
\end{tabular} & $7.0 \mathrm{E}+00$ \\
\hline & 9 & {$\left[\mathrm{C}_{13} \mathrm{H}_{2} \mathrm{ClN}_{3}\right]^{+}$} & 234.9981 & 234.9937 & 1020 & 3.9 & 931 & 40.49 & 38 & 3 & 39 & \begin{tabular}{|l|}
0.45 \\
\end{tabular} & $7.0 \mathrm{E}+00$ \\
\hline & 10 & {$\left[\mathrm{C}_{6} \mathrm{H}_{10} \mathrm{BrN}_{3} \mathrm{O}_{2}\right]^{+}$} & 234.9927 & 234.9957 & 960 & 3.2 & 902 & 40.51 & 42 & 3 & 33 & 0.52 & $6.0 \mathrm{E}+00$ \\
\hline
\end{tabular}




\begin{tabular}{|c|c|c|c|c|c|c|c|c|c|c|c|c|c|}
\hline \multicolumn{14}{|c|}{ Monochlorodimethylpropylphenol $\left(\mathrm{C}_{11} \mathrm{H}_{15} \mathrm{ClO}\right)$} \\
\hline \multirow{3}{*}{ Isomer 1} & 1 & {$\left[\mathrm{C}_{10} \mathrm{H}_{15} \mathrm{ClN}_{2}\right]^{+}$} & 198.0888 & 198.0924 & 43176 & 2.8 & 961 & 27.18 & 2226 & 24 & 73 & 0.24 & $2.8 \mathrm{E}+03$ \\
\hline & 2 & {$\left[\mathrm{C}_{11} \mathrm{H}_{15} \mathrm{ClO}\right]^{+}$} & 198.0886 & 198.0812 & 36241 & 6.4 & 959 & 27.17 & 1889 & 13 & 41 & 0.18 & $1.5 \mathrm{E}+03$ \\
\hline & 3 & {$\left[\mathrm{C}_{9} \mathrm{H}_{13} \mathrm{ClN}_{3}\right]^{+}$} & 198.0896 & 198.0799 & 23816 & 7.4 & 957 & 27.19 & 1428 & 8 & 26 & 0.31 & $5.7 \mathrm{E}+02$ \\
\hline \multirow{2}{*}{ Isomer 2} & 1 & {$\left[\mathrm{C}_{11} \mathrm{H}_{15} \mathrm{ClO}\right]^{+}$} & 198.0890 & 198.0812 & 4306 & 5.3 & 981 & 29.30 & 199 & 5 & 32 & 0.40 & $2.8 \mathrm{E}+02$ \\
\hline & 2 & {$\left[\mathrm{C}_{9} \mathrm{H}_{13} \mathrm{ClN}_{3}\right]^{+}$} & 198.0890 & 198.0799 & 4306 & 5.7 & 981 & 29.30 & 199 & 5 & 32 & 0.40 & $2.8 \mathrm{E}+02$ \\
\hline & & & & & & & & & & & & & \\
\hline \multicolumn{2}{|c|}{$\begin{array}{l}\text { Order of the candidate } \\
\text { for the isotopic feature }\end{array}$} & $\begin{array}{l}\text { Candidate } \\
\text { molecular ion } \\
\text { formula }\end{array}$ & $\begin{array}{l}\text { Detected } \\
\text { mass of } \\
\text { molecular } \\
\text { ion }(\mathrm{Da})\end{array}$ & $\begin{array}{l}\text { Exact mass } \\
\text { of molecular } \\
\text { ion (Da) }\end{array}$ & Intensity & $\begin{array}{l}\text { NEME } \\
(\mathrm{mDa})\end{array}$ & $\begin{array}{l}\text { PCS } \\
(\% 0)\end{array}$ & $\begin{array}{l}\text { RT } \\
(\min )\end{array}$ & $\begin{array}{l}\text { Peak } \\
\text { Area }\end{array}$ & $\begin{array}{l}\text { ND } \\
\text { CS }\end{array}$ & $\begin{array}{l}\text { RCS } \\
(\%)\end{array}$ & RPW & $\begin{array}{l}\text { Peak } \\
\text { Identificati } \\
\text { on score }\end{array}$ \\
\hline \multirow{5}{*}{ Isomer 3} & 1 & {$\left[\mathrm{C}_{12} \mathrm{H}_{15} \mathrm{Cl}\right]^{+}$} & 194.0898 & 194.0863 & 178233 & 4.4 & 959 & 31.91 & 11550 & 26 & 44 & 0.18 & $4.3 \mathrm{E}+03$ \\
\hline & 2 & {$\left[\mathrm{C}_{11} \mathrm{H}_{16} \mathrm{ClN}\right]^{+}$} & 197.0876 & 197.0972 & 20262 & 7.4 & 988 & 31.73 & 1339 & 14 & 46 & 0.38 & $2.0 \mathrm{E}+03$ \\
\hline & 3 & {$\left[\mathrm{C}_{10} \mathrm{H}_{14} \mathrm{ClN}_{2}\right]^{+}$} & 197.0876 & 197.0846 & 20262 & 2.0 & 962 & 31.73 & 1814 & 21 & 37 & 0.29 & $1.4 \mathrm{E}+03$ \\
\hline & 4 & {$\left[\mathrm{C}_{11} \mathrm{H}_{15} \mathrm{ClO}\right]^{+}$} & 198.0869 & 198.0812 & 4252 & 4.0 & 981 & 31.82 & 278 & 5 & 25 & 0.54 & $2.4 \mathrm{E}+02$ \\
\hline & 5 & {$\left[\mathrm{C}_{9} \mathrm{H}_{13} \mathrm{ClN}_{3}\right]^{+}$} & 198.0869 & 198.0799 & 4252 & 5.0 & 979 & 31.82 & 228 & 4 & 24 & 0.53 & $1.9 \mathrm{E}+02$ \\
\hline & & & & & & & & & & & & & \\
\hline \multirow{3}{*}{ Isomer 4} & 1 & {$\left[\mathrm{C}_{10} \mathrm{H}_{15} \mathrm{ClN}_{2}\right]^{+}$} & 198.0887 & 198.0924 & 17002 & 4.4 & 953 & 32.14 & 791 & 8 & 46 & 0.47 & $4.5 \mathrm{E}+02$ \\
\hline & 2 & {$\left[\mathrm{C}_{11} \mathrm{H}_{15} \mathrm{ClO}\right]^{+}$} & 198.0891 & 198.0812 & 13553 & 6.5 & 961 & 32.13 & 873 & 6 & 23 & 0.39 & \begin{tabular}{|l|}
$3.6 \mathrm{E}+02$ \\
\end{tabular} \\
\hline & 3 & {$\left[\mathrm{C}_{9} \mathrm{H}_{13} \mathrm{ClN}_{3}\right]^{+}$} & 198.0895 & 198.0799 & 8891 & 6.6 & 963 & 32.12 & 699 & 5 & 19 & 0.53 & $2.2 \mathrm{E}+02$ \\
\hline \multicolumn{14}{|c|}{ Monochloromethoxyphenol $\left(\mathrm{C}_{7} \mathrm{H}_{7} \mathrm{ClO}_{2}\right)$} \\
\hline \multirow{10}{*}{ Isomer 1} & 1 & {$\left[\mathrm{C}_{5} \mathrm{H}_{5} \mathrm{ClN}_{3} \mathrm{O}\right]^{+}$} & 158.0075 & 158.0121 & 3343600 & 4.4 & 995 & 16.39 & 330542 & 357 & 80 & 0.03 & $3.3 \mathrm{E}+05$ \\
\hline & 2 & {$\left[\mathrm{C}_{7} \mathrm{H}_{7} \mathrm{ClO}_{2}\right]^{+}$} & 158.0075 & 158.0135 & 3343600 & 5.8 & 997 & 16.39 & 330539 & 344 & 78 & 0.03 & $3.2 \mathrm{E}+05$ \\
\hline & 3 & {$\left[\mathrm{C}_{7} \mathrm{H}_{8} \mathrm{ClP}\right]^{+}$} & 158.0075 & 158.0053 & 3343600 & 2.6 & 997 & 16.39 & 330505 & 308 & 70 & 0.03 & $3.1 \mathrm{E}+05$ \\
\hline & 4 & {$\left[\mathrm{C}_{6} \mathrm{H}_{5} \mathrm{ClNO}_{2}\right]^{+}$} & 158.0075 & 158.0009 & 3343600 & 6.9 & 996 & 16.39 & 330154 & 199 & 54 & 0.04 & $1.7 \mathrm{E}+05$ \\
\hline & 5 & {$\left[\mathrm{C}_{10} \mathrm{H}_{5} \mathrm{Cl}\right]^{+}$} & 160.0043 & 160.0080 & 1410274 & 4.4 & 954 & 16.39 & 125002 & 131 & 66 & 0.07 & $3.0 \mathrm{E}+04$ \\
\hline & 6 & {$\left[\mathrm{C}_{10} \mathrm{H}_{6} \mathrm{Cl}\right]^{+}$} & 161.0093 & 161.0158 & 162034 & 5.1 & 951 & 16.39 & 14282 & 49 & 58 & 0.16 & $5.7 \mathrm{E}+03$ \\
\hline & 7 & {$\left[\mathrm{C}_{9} \mathrm{H}_{4} \mathrm{ClN}\right]^{+}$} & 161.0093 & 161.0032 & 162034 & 4.8 & 952 & 16.39 & 14121 & 37 & 50 & 0.19 & $4.3 \mathrm{E}+03$ \\
\hline & 8 & {$\left[\mathrm{C}_{5} \mathrm{H}_{6} \mathrm{ClN}_{2} \mathrm{O}_{2}\right]^{+}$} & 161.0101 & 161.0118 & 52631 & 1.9 & 954 & 16.44 & 3723 & 39 & 71 & 0.19 & $3.9 \mathrm{E}+03$ \\
\hline & 9 & {$\left[\mathrm{C}_{6} \mathrm{H}_{9} \mathrm{ClNP}\right]^{+}$} & 161.0104 & 161.0162 & 27550 & 3.9 & 954 & 16.45 & 2016 & 32 & 62 & 0.20 & $2.3 \mathrm{E}+03$ \\
\hline & 10 & {$\left[\mathrm{C}_{9} \mathrm{H}_{3} \mathrm{ClN}\right]^{+}$} & 160.0046 & 159.9954 & 241690 & 8.7 & 953 & 16.45 & 19076 & 13 & 33 & 0.37 & $1.6 \mathrm{E}+03$ \\
\hline \multicolumn{14}{|c|}{ 2-Bromo-4-methoxy-phenol $\left(\mathrm{C}_{7} \mathrm{H}_{7} \mathrm{BrO}_{2}\right)$} \\
\hline \multirow{3}{*}{ Isomer 1} & 1 & {$\left[\mathrm{C}_{5} \mathrm{H}_{5} \mathrm{BrN}_{3} \mathrm{O}\right]^{+}$} & 201.9601 & 201.9616 & 921291 & 1.0 & 998 & 18.60 & 50141 & 72 & 89 & 0.09 & $1.7 \mathrm{E}+05$ \\
\hline & 2 & {$\left[\mathrm{C}_{7} \mathrm{H}_{7} \mathrm{BrO}_{2}\right]^{+}$} & 201.9601 & 201.9630 & 921291 & 2.1 & 999 & 18.60 & 50141 & 72 & 89 & 0.09 & $1.6 \mathrm{E}+05$ \\
\hline & 3 & {$\left[\mathrm{C}_{7} \mathrm{H}_{8} \mathrm{BrP}\right]^{+}$} & 201.9601 & 201.9548 & 921291 & 6.3 & 999 & 18.60 & 50077 & 49 & 65 & 0.12 & $9.0 \mathrm{E}+04$ \\
\hline
\end{tabular}




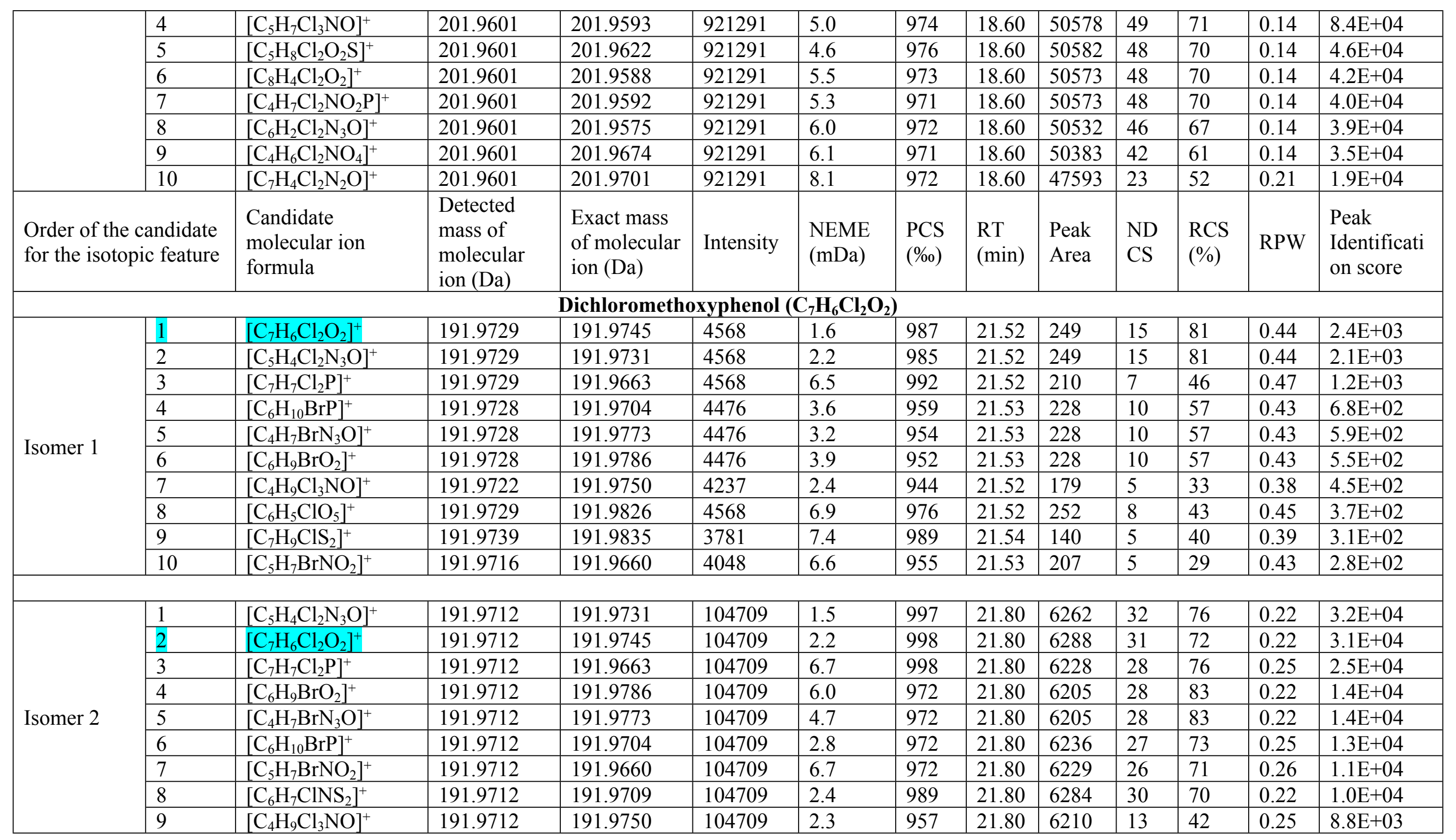


Table S.13. Comparison between positive and negative APGC-QToF for the suggested novel contaminants in LMLT $2014(\mathrm{GC} \times \mathrm{GC}-\mathrm{HRT})^{9}$

\begin{tabular}{|c|c|c|}
\hline Compound & $\begin{array}{c}\text { Negative APGC } \\
\text { Relative retention time a }\end{array}$ & $\begin{array}{c}\text { Positive APGC } \\
\text { Relative retention time a }\end{array}$ \\
\hline $\mathrm{C}_{12} \mathrm{H}_{19} \mathrm{ClO}$ & $0.70,0.71$ & $0.86,0.94$ \\
\hline $\mathrm{C}_{9} \mathrm{H}_{11} \mathrm{ClO}_{2}$ & ND & $0.47,1.12$ \\
\hline $\mathrm{C}_{9} \mathrm{H}_{13} \mathrm{ClO}_{2}$ & 0.56 & $0.49,0.95,1.02$ \\
\hline $\mathrm{C}_{6} \mathrm{H}_{8} \mathrm{ClO}$ & ND & $0.28,0.47,0.58,0.59$ \\
\hline $\mathrm{C}_{8} \mathrm{H}_{9} \mathrm{ClO}_{2}$ & $0.49,1.01,1.11$ & $0.561 .0 .567,0.68,1.06$ \\
\hline $\mathrm{C}_{9} \mathrm{H}_{11} \mathrm{ClO}_{2}$ & ND & $0.47,1.12$ \\
\hline $\mathrm{C}_{8} \mathrm{H}_{9} \mathrm{ClO}_{2}$ & $0.49,1.01,1.11$ & $0.561,0.567,0.68,1.06$ \\
\hline $\mathrm{C}_{11} \mathrm{H}_{17} \mathrm{ClO}_{2}$ & $0.561,0.569,1.18$ & $1.01,1.03,1.04$ \\
\hline $\mathrm{C}_{7} \mathrm{H}_{6} \mathrm{BrClO}_{2}$ & ND & 0.72 \\
\hline $\mathrm{C}_{8} \mathrm{H}_{6} \mathrm{Cl}_{4} \mathrm{O}_{2}$ (tetrachloro dimethoxybenzene) & 0.84 & 0.84 \\
\hline $\mathrm{C}_{10} \mathrm{H}_{7} \mathrm{ClN}_{2}$ (chlorophenyl-pyrimidine) & 0.92 & $0.80,0.86$ \\
\hline $\mathrm{C}_{10} \mathrm{H}_{4} \mathrm{Cl}_{7} \mathrm{O}$ & ND & 1.05 \\
\hline $\mathrm{C}_{13} \mathrm{H}_{8} \mathrm{Cl}_{2} \mathrm{O}$ (dichlorobenzophenone) & 1.1213 & ND \\
\hline $\mathrm{C}_{14} \mathrm{H}_{13} \mathrm{ClO}_{4}$ & ND & $1.14,1.17$ \\
\hline $\mathrm{C}_{12} \mathrm{H}_{7} \mathrm{Cl}_{2} \mathrm{~N}$ (dichloro-9H-carbazole) & ND & 1.22 \\
\hline $\mathrm{C}_{7} \mathrm{H}_{7} \mathrm{ClO}_{2}$ (Monochloromethoxyphenol) & $0.43,0.45,0.91$ & 0.49 \\
\hline $\mathrm{C}_{7} \mathrm{H}_{6} \mathrm{Cl}_{2} \mathrm{O}_{2}$ (Dichloromethoxyphenol) & 0.49 & $0.64,0.65$ \\
\hline $\mathrm{C}_{7} \mathrm{H}_{7} \mathrm{BrO}_{2}$ (2-Bromo-4-methoxy-phenol) & ND & 0.56 \\
\hline $\mathrm{C}_{11} \mathrm{H}_{15} \mathrm{ClO}$ (Monochlorodimethylpropylphenol) & $0.65,0.67,0.72$ & $0.81,0.88,0.960,0.967$ \\
\hline
\end{tabular}

a The relative retention index was derived using the retention time of the injection standard (PCB-65) 


\section{S.13. Checking Candidate Compounds against CompTox Chemicals Dashboard}

The CompTox Chemicals Dashboard database (DSSTox MS Ready) posted on 11/14/2016 contains around 620 thousand molecular formulas structures. Checking the availability of candidate compounds in this database may simplify the algorithm data reduction complexity by suggesting a list of candidate compounds that have a reasonable peak shape and at least one candidate structure record. The Comptox chemistry dashboard library is sensitive to the order of the elements in the compound molecular formula. The Hill notation system ${ }^{10}$ is applied to develop molecular formulas for this module. In the Hill notation system, the first element is carbon is followed by hydrogen and subsequently other elements based on their alphabetic order. ${ }^{10}$ Therefore, molecular formulas should be arranged as " $\mathrm{C}_{c} \mathrm{H}_{h} \mathrm{Br}_{b r} \mathrm{Cl}_{c l} \mathrm{~N}_{n} \mathrm{O}_{o} \mathrm{P}_{p} \mathrm{~S}_{s}$ ". 


\section{References}

(1) Crimmins, B.; Xia, X.; Hopke, P.; Holsen, T. Anal. Bioanal. Chem. 2014, 406, 1471-1480.

(2) ACD/Spectrus, version 2015.2.5, Advanced Chemistry Development, Inc., Toronto, ON, Canada, www.acdlabs.com, 2015.

(3) Fakouri Baygi, S.; Crimmins, B.S.; Hopke, P.K.; Holsen, T.M. Environ. Sci. Technol. 2016, 50, 9460-9468.

(4) Riazi, M.R.; Daubert, T.E. Ind. Eng. Chem. Process Des. Dev. 1980, 19, 289-294.

(5) Riazi, M.R.; Daubert, T.E. Ind. Eng. Chem. Res. 1987, 26, 755-759.

(6) Simpson, S.; Gross, M.S.; Olson, J.R.; Zurek, E.; Aga, D.S. Anal. Chem. 2015, 87, 22992305.

(7) IARC Working Group on the Evaluation of Carcinogenic Risk to Humans. Polychlorinated Biphenyls and Polybrominated Biphenyls. Lyon (FR): International Agency for Research on Cancer; 2016. (IARC Monographs on the Evaluation of Carcinogenic Risks to Humans, No. 107.)

(8) Myers, A. L.; Jobst, K. J.; Mabury, S. A.; Reiner, E. J. J. Mass Spectrom. 2014, 49 (4), 291-296.

(9) Fernando, S.; Renaguli, A.; Milligan, M.S.; Pagano, J.J.; Hopke, P.K.; Holsen, T.M.;

Crimmins, B.S. Environ. Sci. Technol. 2018, 52, 2909-2917.

(10) Hill, E.A. J. Am. Chem. Soc. 1900, 22, 478-494. 